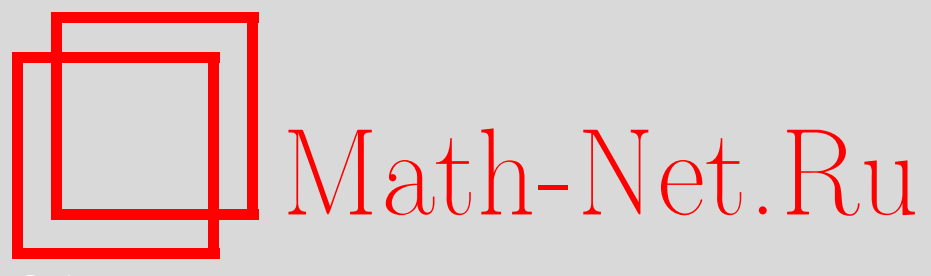

М. Е. Жуковский, А. М. Райгородский, Случайные графы: модели и предельные характеристики, УМН, 2015, том 70, выпуск 1, 35-88

DOI: https://doi.org/10.4213/rm9626

Использование Общероссийского математического портала Math-Net.Ru подразумевает, что вы прочитали и согласны с пользовательским соглашением http://www . mathnet.ru/rus/agreement

Параметры загрузки:

IP : 18.207 .199 .55

26 апреля 2023 г., 12:13:44

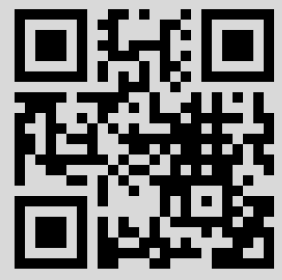




\title{
Случайные графы: модели и предельные характеристики
}

\author{
М. Е. Жуковский, А. М. Райгородский
}

В настоящей статье представлен обзор известных результатов в области предельного поведения вероятностей свойств первого порядка случайных графов. Совокупность результатов, приведенных в статье, относится к законам нуля или единицы для свойств случайных графов. Мы сконцентрируемся на модели Эрдёша-Реньи случайного графа и рассмотрим также некоторые обобщения этой модели, мотивированные задачами теории кодирования и комбинаторной геометрии.

Библиография: 65 названий.

Ключевые слова: случайные графы, дистанционные графы, предельные теоремы, законы нуля или единицы, свойства первого порядка.

DOI: $10.4213 / \mathrm{rm} 9626$

\section{СОДЕРЖАНИЕ}

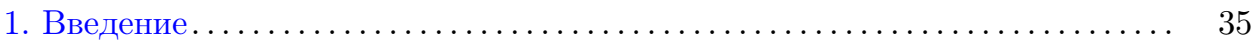

2. Случайные графы в модели Эрдёша-Реньи и их простейшие свойства. 36

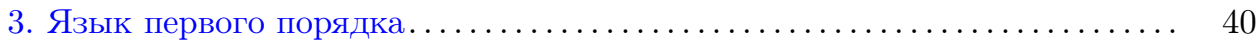

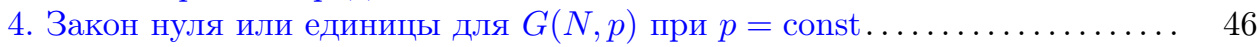

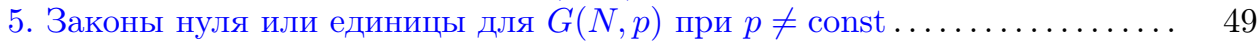

6. Некоторые обобщения результатов из разделов 4 и $5 \ldots \ldots \ldots \ldots \ldots \ldots \ldots . \ldots$

7. Ограничение кванторной глубины .......................... 59

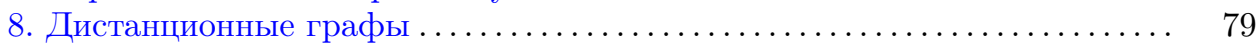

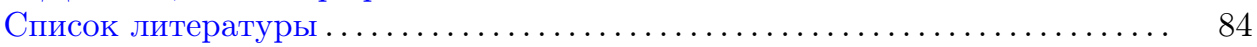

\section{1. Введение}

Понятие о случайном графе является сейчас одним из центральных в дискретной математике. Однако до середины 50-х годов XX в. систематической теории случайных графов не было. Были лишь разрозненные работы, в которых случайные графы так или иначе возникали в качестве инструмента (см.,

Работа выполнена при поддержке РФФИ (гранты № 13-01-00612, 15-01-00350), грантов Президента РФ МД-6277.2013.1, MK-2184.2014.1 и программы "Ведущие научные школы" (грант НШ-2519.2012.1).

(C) М. Е. ЖКуковский, А. М. РАйгородский, 2015 
например, [1]-[3]). И лишь классические статьи [4]-[6] П. Эрдёша и А. Реньи, опубликованные на рубеже 50-х и 60-х годов, заложили основы современной науки о случайных графах. За прошедшие полвека теория случайных графов выросла в мощную и бурно развивающуюся дисциплину, богатую как фундаментальными результатами, так и приложениями в различных областях математики, информатики, биологии и т. д.

В широком смысле этого слова случайный граф - это случайный элемент, принимающий значения в некотором множестве графов и имеющий заданное распределение. K настоящему времени глубоко исследован целый ряд моделей случайного графа - от классической модели Эрдёша-Реньи и ее естественных обобщений до моделей веб-графов, социальных, биологических сетей и т. д. (см. [7]-[16]).

В настоящем обзоре мы сконцентрируемся на модели Эрдёша-Реньи случайного графа. Также мы рассмотрим некоторые обобщения этой модели, мотивированные задачами теории кодирования и комбинаторной геометрии. При этом мы, разумеется, не станем обсуждать все многообразие результатов, полученных в области за пять десятилетий, но рассмотрим исключительно важный специальный пласт фактов, совокупность которых правильнее всего характеризовать выражением “законы нуля или единицы для свойств случайных графов".

В последующих разделах мы раскроем смысл всех терминов, которые мы пока что употребляли без точных определений.

\section{2. Случайные графы в модели Эрдёша-Реньи и их простейшие свойства}

Прежде всего напомним определение случайного графа в биномиальной модели Эрдёша-Реньи. Пусть $N \in \mathbb{N}, 0 \leqslant p \leqslant 1$. Рассмотрим множество $\Omega_{N}=\left\{G=\left(V_{N}, E\right)\right\}$ всех неориентированных графов без петель и кратных ребер с множеством вершин $V_{N}=\{1, \ldots, N\}$. Случайный граф в модели

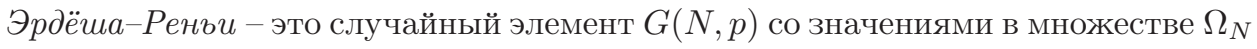
и распределением $\mathrm{P}_{N, p}$ на $\mathscr{F}_{N}=2^{\Omega_{N}}$, определенным формулой

$$
\mathrm{P}_{N, p}(G)=p^{|E|}(1-p)^{C_{N}^{2}-|E|} .
$$

Иными словами, любые две различные вершины графа $G(N, p)$ соединены ребром с вероятностью $p$ независимо от всех остальных пар вершин. В дальнейшем мы будем рассматривать модели, в которых вероятность $p$ зависит от количества вершин $N$ (в этом случае для вероятностной меры мы по-прежнему будем использовать обозначение $\mathrm{P}_{N, p}$ вместо $\left.\mathrm{P}_{N, p(N)}\right)$, причем нас будет интересовать асимптотическое поведение вероятностей свойств случайных графов при $N \rightarrow \infty$.

Случайный граф Эрдёша-Реньи является частным случаем более общей модели, называемой случайнъм подграфом (см., например, [8]-[11]). Пусть $H=(V, E)$ - произвольный граф без петель и кратных ребер, $0 \leqslant p \leqslant 1$. Рассмотрим множество $\Omega_{H}=\{\widetilde{H}=(V, \widetilde{E}), \widetilde{E} \subseteq E\}$ всех остовных подграфов графа $H$. Случайным подграфом графа $H$ называется случайный элемент $\mathscr{G}(H, p)$ 
со значениями в множестве $\Omega_{H}$ и распределением $\mathrm{P}_{H, p}$ на $\mathscr{F}_{H}=2^{\Omega_{H}}$, определенным формулой

$$
\mathrm{P}_{H, p}(\widetilde{H})=p^{|\widetilde{E}|}(1-p)^{|E|-|\widetilde{E}|} .
$$

Очевидно, что если $H$ - полный граф на $N$ вершинах, то $\mathscr{G}(H, p)=G(N, p)$.

Именно Эрдёш и Реньи установили, что свойства случайного графа $G(N, p)$, рассматриваемые ими в первых работах по случайным графам, "возникают" в каком-то смысле внезапно. Например, для каждого из свойств "содержать клику фиксированного размера", “быть связным", "содержать 'гигантскую' компоненту" (т. е. компоненту связности, количество вершин в которой не меньше $c N$ с одной и той же константой $c$ для каждого $N$ ) найдется функция $p_{0}=p_{0}(N)$, для которой это свойство не выполнено с вероятностью, стремящейся к 1 при $N \rightarrow \infty$, если $p=o\left(p_{0}\right)$ при $N \rightarrow \infty$, и выполнено с вероятностью, стремящейся к 1 при $N \rightarrow \infty$, если, напротив, $p_{0}=o(p)$ при $N \rightarrow \infty$. Разумеется, для некоторых свойств имеют место и симметричные ситуации: при $p=o\left(p_{0}\right)$ свойство выполнено с предельной вероятностью 1 , а при $p_{0}=o(p)-$ не выполнено с аналогичной вероятностью. В любом случае такая функция $p_{0}$ называется пороговой для данного свойства. Скажем, для свойства "содержать гигантскую компоненту" функция $1 / N$ является пороговой (см. [8]-[11]).

Здесь важно заметить, что все перечисленные свойства графов являются монотонными. Свойство называется монотонным, если выполнено одно из двух утверждений:

- для любых графов $H \subseteq G$ из того, что граф $H$ обладает этим свойством, следует, что граф $G$ также обладает этим свойством (в этом случае свойство называется возрастающим);

- для любых графов $H \subseteq G$ из того, что граф $G$ обладает этим свойством, следует, что граф $H$ также обладает этим свойством (в этом случае свойство называется убывающим).

Б. Боллобаш и А. Томасон в 1987 г. доказали, что у каждого монотонного свойства существует пороговая функция. Более того, если выше речь шла лишь о графах $G(N, p)$, то в теореме, которую мы приводим ниже, рассматриваются случайные подграфы $\mathscr{G}\left(G_{n}, p\right)$ графов $G_{n}$ из практически произвольной последовательности $\left\{G_{n}\right\}_{n \in \mathbb{N}}$.

Tеорема 1 (Б. Боллобаш, А. Томасон, 1987, [17]). Пусть L - некоторое возрастающее свойство графов. Пусть, кроме того, $\left\{G_{n}\right\}_{n \in \mathbb{N}}-$ такая последовательность графов, что $\left|V\left(G_{n}\right)\right| \rightarrow \infty$ при $n \rightarrow \infty$. Тогда существует такая функиия $p_{0}=p_{0}(n)$, что если $p=o\left(p_{0}\right)$, то $\mathrm{P}_{G_{n}, p}(L) \rightarrow 0, n \rightarrow \infty$, a если $p_{0}=o(p)$, mo $\mathrm{P}_{G_{n}, p}(L) \rightarrow 1, n \rightarrow \infty$.

Аналогичный результат верен и для убывающих свойств (асимптотические вероятности 0 и 1 меняются местами). Иными словами, теорема 1 дает весьма общий признак существования пороговых функций для свойств случайных подграфов $\mathscr{G}\left(G_{n}, p\right)$.

Теорема 1 делает заведомо непустым следующее общее рассуждение. Пусть $\left\{G_{n}\right\}_{n \in \mathbb{N}}-$ некоторая последовательность графов, а $\mathscr{C}$ - некоторый класс свойств, для которых у случайных подграфов $\mathscr{G}\left(G_{n}, p\right)$ существуют пороговые функции. 
Пусть, кроме того, $\mathscr{P}^{0}$ - класс всех пороговых функций этих свойств. Тогда если $p=p(n)$ - такая функция, что

$$
\forall p_{0} \in \mathscr{P}^{0} \quad\left(p=o\left(p_{0}\right)\right) \vee\left(p_{0}=o(p)\right),
$$

то для графа $\mathscr{G}\left(G_{n}, p\right)$ справедлив закон нуля или единицы для класса свойств $\mathscr{C}$, т. е. для любого свойства из $\mathscr{C}$ вероятность того, что случайный граф $\mathscr{G}\left(G_{n}, p\right)$ обладает этим свойством, стремится либо к 0, либо к 1. Этот факт заставил многих авторов заняться поиском для различных последовательностей графов $\left\{G_{n}\right\}_{n \in \mathbb{N}}$ и различных классов свойств $\mathscr{C}$ таких множеств функций $\mathscr{P}_{\mathscr{C}}$, что для любого $p \in \mathscr{P}_{\mathscr{C}}$ случайный граф $\mathscr{G}\left(G_{n}, p\right)$ подчиняется закону нуля или единицы. Именно такого рода задачам мы посвятим основную часть обзора. Однако, желая дать дополнительную мотивировку нашему исследованию, мы продолжим ниже обсуждение пороговых функций для случайного графа $G(N, p)$.

Итак, вернемся к случайному графу $G(N, p)$. В 1960 г. П. Эрдёш и А. Реньи доказали теорему о существовании пороговой функции для свойства " $G(N, p)$ содержит копию данного сбалансированного графа" (определение сбалансированного графа дано ниже в этом абзаце), которая позже была обобщена А. Ручинским и А. Винсом на случай произвольного (не обязательно сбалансированного) графа. Рассмотрим произвольный граф $G$. В дальнейшем мы будем обозначать $v(G)$ количество вершин графа $G$ и $e(G)$ количество его ребер. Назовем отношение $\rho(G)=e(G) / v(G)$ плотностью графа $G$. Граф $G$ называется сбалансированным, если для каждого его подграфа $H$ выполнено неравенство $\rho(H) \leqslant \rho(G)$.

Теорема 2 (П. Эрдёш, А. Реньи, 1960, [5]). Пусть $G$ - сбалансированный граф. Тогда функиия $p=N^{-1 / \rho(G)}$ является пороговой для графа $G(N, p)$ и свойства содержать копию графа $G$.

Пусть теперь $G$ - произвольный граф. Положим

$$
\rho^{\max }(G)=\max _{H \subseteq G} \rho(H) .
$$

Tеорема 3 (А. Ручински, А. Винс, 1985, [18]). Функиия $p=N^{-1 / \rho^{\max }(G)}$ является пороговой для графа $G(N, p)$ и свойства содержать копию графа $G$.

Кроме того, в 1981 г. Б. Боллобаш нашел асимптотическое распределение количества копий в $G(N, p)$ фиксированного строго сбалансированного графа в случае, когда вероятность проведения ребра равна пороговой вероятности появления копии рассматриваемого графа (напомним, что строго сбалансированным графом называется сбалансированный граф, плотность которого строго больше плотностей всех его собственных подграфов). Здесь и далее для произвольного графа $G$ мы будем обозначать $N_{G}$ количество копий $G$ в случайном графе $G(N, p)$ (с точностью до перенумерации вершин). Пусть $G-$ строго сбалансированный граф. 
Теорема 4 (Б. Боллобаш, 1981, [19]). Пусть а - количество автоморфизмов графа $G, p=N^{-1 / \rho(G)}$. Тогда

$$
N_{G} \stackrel{d}{\rightarrow} \operatorname{Pois}(1 / a), \quad N \rightarrow \infty .
$$

Здесь Pois(1/a) - пуассоновская случайная величина со средним $1 /$ a.

Обозначим $L_{G}=\left\{N_{G}>0\right\}$ свойство содержать копию графа $G$. Пусть функции $p$ и $1-p$ меняются медленнее, чем любая степенная. Иными словами, для любого положительного $\alpha$ имеем

$$
\min \{p, 1-p\} N^{\alpha} \rightarrow \infty, \quad N \rightarrow \infty
$$

Положим

$$
\mathscr{L}_{0}=\bigcup_{n \in \mathbb{N}} \bigcup_{G \in \Omega_{n}}\left\{L_{G}, \overline{L_{G}}\right\} .
$$

Тогда из теоремы 3 следует, что для любого $L \in \mathscr{L}_{0}$ либо $\mathrm{P}_{N, p}(L) \rightarrow 1, N \rightarrow \infty$, либо $\mathrm{P}_{N, p}(L) \rightarrow 0, N \rightarrow \infty$. Иными словами, для класса свойств $\mathscr{L}_{0}$ случайный граф $G(N, p)$ подчиняется закону нуля или единицы. Очевидно, тот же вывод можно сделать и для $p=N^{-\alpha}$, где $\alpha$ - положительное иррациональное число. В то же время несложно доказать (см., например, [20]), что для любого ращионального числа $\alpha \in(0,1]$ существует строго сбалансированный граф с плотностью $1 / \alpha$. Поэтому в силу теоремы 4 случайный граф $G\left(N, N^{-\alpha}\right)$ не подчиняется закону нуля или единицы для класса свойств $\mathscr{L}_{0}$ при $\alpha \in(0,1] \cap \mathbb{Q}$.

Рассмотренные свойства могут быть записаны с помощью логических формул. Например, пусть $G$ - полный граф на трех вершинах. Тогда свойство $L_{G}$ может быть выражено формулой

$$
\exists x_{1} \exists x_{2} \exists x_{3} \quad\left(x_{1} \sim x_{2}\right) \wedge\left(x_{1} \sim x_{3}\right) \wedge\left(x_{2} \sim x_{3}\right) .
$$

Эта формула является формулой первого порядка, определение которой мы напомним в следующем разделе. Оказывается, законы нуля или единицы для класса $\mathscr{L}_{0}$ могут быть обобщены на класс всех свойств, выражаемых формулами первого порядка.

В настоящей работе мы также рассматриваем один специальный случай модели случайных подграфов - так называемый случайный дистаниионный граф. Рассмотрение дистанционных графов мотивировано классической задачей комбинаторной геометрии о хроматическом числе пространства (см. [21]-[28]). Впервые полный дистанционный граф, определение которого сформулировано ниже и свойства которого изучаются в данной работе, в геометрическом контексте рассмотрели в 1981 г. П. Франкл и Р. М. Уилсон. С помощью этого графа они показали, что хроматическое число пространства $\mathbb{R}^{n}$ растет экспоненциально (см. [29]). В 1991 г. Дж. Кан и Г. Калаи применили результаты Франкла и Уилсона для опровержения классической гипотезы Борсука о том, что всякое ограниченное неодноточечное множество в $\mathbb{R}^{n}$ может быть разбито на $n+1$ часть меньшего диаметра (см. [21] и [30]). Таким образом, изучение внутренней структуры дистанционного графа и его подграфов играет исключительно 
важную роль. Наши результаты показывают, в частности, что любой подграф либо содержится в почти всех дистанционных графах, полученных из полного дистанционного графа удалением ребер, либо не содержится в почти всех таких дистанционных графах. Сейчас с исследованием дистанционных графов связаны одни из самых широко изучаемых разделов комбинаторной геометрии (см. [21]-[28], [31]). Отметим, что в то же время некоторые из этих графов изучаются и в теории кодирования (см. [32]-[34]).

Напомним определение случайного дистанционного графа (см. [35]-[45]). Пусть $M$ - произвольное конечное множество целых чисел, $\left\{n_{i}\right\}_{i \in \mathbb{N}}$ - возрастающая последовательность натуральных чисел. Пусть, кроме того, функции $a_{m}:\left\{n_{i}\right\}_{i \in \mathbb{N}} \rightarrow \mathbb{Z}_{+}, m \in M$, таковы, что при всех $n \in\left\{n_{i}\right\}_{i \in \mathbb{N}}$ выполнено равенство $\sum_{m \in M} a_{m}(n)=n$. Пусть также задана функция $c:\left\{n_{i}\right\}_{i \in \mathbb{N}} \rightarrow \mathbb{Z}$. При всех $n \in\left\{n_{i}\right\}_{i \in \mathbb{N}}$ определим дистаниионный граф $G_{n}=\left(V_{n}, E_{n}\right)$ с множеством вершин $V_{n}$, состоящим из всех $n$-мерных векторов, которые имеют по $a_{m}(n)$ координат, равных $m$ для каждого $m \in M$, т. е.

$$
\begin{aligned}
V_{n}=\left\{\mathbf{v}=\left(v^{1}, \ldots, v^{n}\right): \forall i \in\{1, \ldots, n\} v^{i} \in M ;\right. \\
\left.\forall m \in M\left|\left\{i \in\{1, \ldots, n\}: v^{i}=m\right\}\right|=a_{m}(n)\right\},
\end{aligned}
$$

и множеством ребер

$$
E_{n}=\left\{\{\mathbf{u}, \mathbf{v}\} \in V_{n} \times V_{n}:\langle\mathbf{u}, \mathbf{v}\rangle=c(n)\right\},
$$

где $\langle\mathbf{u}, \mathbf{v}\rangle$ - евклидово скалярное произведение. Пусть $p: \mathbb{N} \rightarrow[0,1]$. Случайнъц дистаниионным графом называется случайный подграф $\mathscr{G}\left(G_{n}, p\right)$ графа $G_{n}$.

В простейшем случае симметричного $\{0,1\}$-дистанционного графа, т. е. при $n_{i}=4 i, M=\{0,1\}, a_{0}=a_{1}=n / 2, c=n / 4$, известны пороговые вероятности для свойств из класса $\mathscr{L}_{0}$. Пусть $\left\{G_{n}\right\}_{n \in 4 \mathbb{N}}$ - последовательность симметричных $\{0,1\}$-дистанционных графов. Для каждого $n \in 4 \mathbb{N}$ обозначим $N=N(n)$ количество вершин графа $G_{n}$, т. е. $N=C_{n}^{n / 2}$. Пусть $G$ - произвольный строго сбалансированный граф.

Tеорема 5 (М.Е. Жуковский, 2012, [41]). Функиия $p=N^{-1 / \rho(G)} \sqrt{\log N}$ является пороговой для графа $\mathscr{G}\left(G_{4 i}, p\right)$ и свойства содержать копию графа $G$.

Ввиду аналогичных результатов для случайного графа Эрдёша-Реньи эта теорема мотивирует следующий вопрос. Если функция $p$ при любом $\alpha>0$ удовлетворяет условию (1), то подчиняется ли рассматриваемый случайный дистанционный граф закону нуля или единицы для класса свойств, выражаемых формулами первого порядка? Ответ на этот вопрос мы даем в разделе 8.

\section{3. Язык первого порядка}

Формуль первого порядка (применительно к свойствам графов) строятся с помощью символов отношения $\sim,=$, логических связок $\neg, \Rightarrow, \Leftrightarrow, \vee, \wedge$, переменных $x, y, x_{1}, \ldots$ (переменные - это вершины графа), кванторов $\forall, \exists$. Символ отношения “ " выражает свойство двух вершин быть соединенными ребром. 
Опишем построение формул подробнее (см. [46], [47]). Введем для этого понятие атома. Это объект, который либо имеет вид $(x \sim y)$, либо имеет вид $(x=y)$, где $x, y$ - переменные. Атом является формулой. Все входящие в атом переменные являются свободными. Ниже мы даем определение связанных и свободных переменных и вместе с этим дальнейшее определение формул первого порядка. Пусть $G$ - некоторый граф (не обязательно конечный). Рассмотрим произвольные вершины $i_{1}, i_{2}$ этого графа. Если $i_{1} \sim i_{2}$, то будем говорить, что формула $(x \sim y)$ истинна для графа $G$ на наборе $\left(i_{1}, i_{2}\right)$. В противном случае будем говорить, что формула ложна. Формула $(x=y)$ истинна только на наборах, состоящих из двух одинаковых вершин, т. е. на наборах $(i, i)$. Иными словами, формула истинна на некотором наборе вершин, если предикат, выражаемый этой формулой, принимает значение 1 на этом наборе. Пусть $\phi, \phi_{1}$, $\phi_{2}$ - формулы, $X, X_{1}, X_{2}$ и $Y, Y_{1}, Y_{2}$ - соответствующие множества свободных и связанных переменных, переменная $x$ принадлежит $X$. Конструкции $\neg \phi$, $\left(\phi_{1} \vee \phi_{2}\right),\left(\phi_{1} \wedge \phi_{2}\right),\left(\phi_{1} \Rightarrow \phi_{2}\right),\left(\phi_{1} \Leftrightarrow \phi_{2}\right),(\forall x \phi),(\exists x \phi)$ являются формулами. При этом $X \backslash\{x\}$ - множество свободных переменных формул $(\forall x \phi),(\exists x \phi)$, а $Y \cup\{x\}$ - множество связанных переменных этих формул, $X_{1} \cup X_{2}$ - множество свободных переменных формул $\left(\phi_{1} \vee \phi_{2}\right),\left(\phi_{1} \wedge \phi_{2}\right),\left(\phi_{1} \Rightarrow \phi_{2}\right),\left(\phi_{1} \Leftrightarrow \phi_{2}\right)$, $Y_{1} \cup Y_{2}$ - множество связанных переменных этих формул, $X$ - множество свободных переменных формулы $(\neg \phi), Y$ - множество связанных переменных этой формулы. Так же как и в случае атома, формула является истинной на некотором наборе вершин, если предикат, выражаемый этой формулой, принимает значение 1 на этом наборе. Замкнутыми называются формулы, не содержащие свободных переменных. Замкнутая формула либо всегда истинна для графа $G$, либо всегда ложна.

Определим кванторную глубину формуль. Глубина атома равна нулю. Глубина формул $\left(\phi_{1} \vee \phi_{2}\right),\left(\phi_{1} \wedge \phi_{2}\right),\left(\phi_{1} \Leftrightarrow \phi_{2}\right),\left(\phi_{1} \Rightarrow \phi_{2}\right)$ равна максимуму глубин формул $\phi_{1}$ и $\phi_{2}$. Глубина формулы $(\neg \phi)$ равна глубине формулы $\phi$. Глубина формул $(\forall x \phi)$ и $(\exists x \phi)$ на единицу больше глубины $\phi$. Замкнутые формулы называются эквивалентными, если они одновременно истинны или одновременно ложны для любого графа $G$.

Приведем примеры формул первого порядка. Формула

$$
\forall x \forall y[(\neg(x=y)) \Rightarrow(x \sim y)]
$$

является замкнутой (обе переменные связанные) и выражает свойство графа быть полным, ее кванторная глубина равна 2. Глубина незамкнутой формулы

$$
\left[\exists x_{3}\left(\left[x_{1} \sim x_{3}\right] \wedge\left[x_{2} \sim x_{3}\right]\right)\right] \vee\left[\forall y_{1} \forall y_{2}\left(\left[y_{1} \sim y_{2}\right] \Rightarrow\left[\left(y_{1} \sim x_{1}\right) \wedge\left(y_{2} \sim x_{2}\right)\right]\right)\right]
$$

также равна 2 , переменные $x_{1}, x_{2}$ являются свободными, остальные - связанными.

В дальнейшем мы будем рассматривать только замкнутые формулы. Если замкнутая формула $\phi$ первого порядка истинна для графа $G$, то будем говорить, что граф $G$ обладает свойством первого порядка $L$, которое определено формулой $\phi$. Под свойством мы подразумеваем множество графов, которые этим свойством обладают. В этой связи множество графов из $\Omega_{N}$, для которых 
истинна формула $\phi$, мы будем обозначать $L^{N}$ или просто $L$, если из контекста ясно, о каком именно количестве вершин идет речь. В дальнейшем, если выполнено равенство $\lim _{N \rightarrow \infty} \mathrm{P}_{N, p}(L)=1$, то будем говорить, что случайный граф c асимптотической вероятностъю 1 обладает свойством $L$. Обозначим $\mathscr{L}$ класс свойств графов, выражаемых формулами первого порядка.

При доказательстве законов нуля или единицы для класса свойств первого порядка используется теорема Эренфойхта. Перед тем как сформулировать ее, напомним некоторые определения. Пусть $k \in \mathbb{N}$. Два графа $G$ и $H$ называются $k$-элементарно эквивалентными, если для любого свойства первого порядка $L$, выражаемого формулой, кванторная глубина которой не превосходит числа $k$, либо $G \in L, H \in L$, либо $G \notin L, H \notin L$. Два графа $G$ и $H$ называются элементарно эквивалентными, если они являются $k$-элементарно эквивалентными для любого натурального числа $k$.

Определим игру Эренфойхта $\operatorname{EHR}(G, H, k)$ на двух графах $G, H$, не обязательно конечных, с двумя игроками (Новатором и Консерватором) и с фиксированным числом раундов $k$ (см. [8], [10], [35]-[40], [46], [48]-[50]). Пусть $V(G)=\left\{x_{1}, \ldots, x_{n}\right\}, V(H)=\left\{y_{1}, \ldots, y_{m}\right\}$. На $\nu$-м ходу $(1 \leqslant \nu \leqslant k)$ Новатор выбирает вершину из любого графа (он выбирает либо $x_{j_{\nu}} \in V(G)$, либо $\left.y_{j_{\nu}^{\prime}} \in V(H)\right)$. Затем Консерватор выбирает вершину из оставшегося графа. Если Новатор выбирает на $\mu$-м ходу, скажем, вершину $x_{j_{\mu}} \in V(G), j_{\mu}=j_{\nu}$ $(\nu<\mu)$, то Консерватор должен выбрать $y_{j_{\nu}^{\prime}} \in V(H)$. Если же на этом ходу Новатор выбирает, скажем, вершину $x_{j_{\mu}} \in V(G), j_{\mu} \notin\left\{j_{1}, \ldots, j_{\mu-1}\right\}$, то и Консерватор должен выбрать такую вершину $y_{j_{\mu}^{\prime}} \in V(H)$, что $j_{\mu}^{\prime} \notin\left\{j_{1}^{\prime}, \ldots, j_{\mu-1}^{\prime}\right\}$. Если он не может этого сделать, то игру выигрывает Новатор. K концу игры выбраны вершины $x_{j_{1}}, \ldots, x_{j_{k}} \in V(G)$, а также вершины $y_{j_{1}^{\prime}}, \ldots, y_{j_{k}^{\prime}} \in V(H)$. Некоторые из этих вершин могут совпадать. Выберем из них только различные: $x_{h_{1}}, \ldots, x_{h_{l}} ; y_{h_{1}^{\prime}}, \ldots, y_{h_{l}^{\prime}}, l \leqslant k$. Консерватор побеждает тогда и только тогда, когда соответствующие подграфы изоморфны:

$$
\left.\left.G\right|_{\left\{x_{h_{1}}, \ldots, x_{h_{l}}\right\}} \cong H\right|_{\left\{y_{h_{1}^{\prime}}, \ldots, y_{h_{l}^{\prime}}\right\}} .
$$

Сформулируем, наконец, теорему Эренфойхта о связи между элементарной эквивалентностью и игрой Эренфойхта.

Теорема 6 (А. Эренфойхт, 1960, [48]). Пусть $G, H$ - два графа, $k$ - натуральное число. Графы $G, H$ являются $k$-элементарно эквивалентными тогда и только тогда, когда у Консерватора есть выигрышная стратегия в игре $\operatorname{EHR}(G, H, k)$.

Из этой теоремы, очевидно, следует, что два графа являются элементарно эквивалентными тогда и только тогда, когда для любого $k \in \mathbb{N}$ у Консерватора есть выигрышная стратегия в игре $\operatorname{EHR}(G, H, k)$.

Для того чтобы продемонстрировать, для каких задач оказывается полезна теорема Эренфойхта, докажем с ее помощью, что свойство связности нельзя записать на языке первого порядка. Предположим противное. Пусть $k-$ глубина формулы первого порядка, с помощью которой можно записать свойство связности. Рассмотрим связный граф $G$, являющийся бесконечной цепью, 
и несвязный граф $H=H_{1} \sqcup H_{2}$, являющийся объединением двух бесконечных цепей $H_{1}$ и $H_{2}$. Такие графы не являются $k$-элементарно эквивалентными в силу предположения. Тогда по теореме 6 у Новатора есть выигрышная стратегия в игре $\operatorname{EHR}(G, H, k)$. Докажем, что это не так.

Введем обозначение, которое мы будем использовать в дальнейшем. Пусть $X$ - произвольный граф, $A$ и $B$ - два его подграфа, $x \in V(A), y \in V(B)$. Обозначим $d_{X}(x, y)$ наименьшее среди количеств ребер в цепях, являющихся подграфами в $X$ и соединяющих вершины $x$ и $y$. Положим

$$
\begin{aligned}
d_{X}(x, B)=d_{X}(B, x) & =\min _{\widetilde{y} \in V(B)} d_{X}(x, \widetilde{y}), \\
d_{X}(A, B) & =\min _{\widetilde{x} \in V(A)} d_{X}(\widetilde{x}, B) .
\end{aligned}
$$

Заметим, что если граф $X$ несвязный, то цепи, соединяющей вершины $x$ и $y$, может и не существовать. Если цепи не существует, то положим $d_{X}(x, y)=\infty$. Для любого натурального числа $n$ мы считаем, что $n<\infty$.

Предположим, что выбранные в первых $i$ раундах, $1 \leqslant i \leqslant k-1$, вершины $x_{1}, \ldots, x_{i} \in V(G), y_{1}, \ldots, y_{i} \in V(H)$ обладают свойством $i$-отделимости, определенным ниже.

Пусть $y_{j_{1}}, \ldots, y_{j_{u}} \in V\left(H_{1}\right), y_{j_{u+1}}, \ldots, y_{j_{i}} \in V\left(H_{2}\right)$, где $j_{1}<\cdots<j_{u}, j_{u+1}<$ $\cdots<j_{i}$ - попарно различные числа. Пусть, кроме того, $\sigma$ - такая перестановка на множестве $\{1, \ldots, i\}$, что вершины $x_{\sigma(1)}, \ldots, x_{\sigma(i)}$ в цепи $G$ следуют подряд (т. е. для любого $j \in\{1, \ldots, i-1\}$ между вершинами $x_{\sigma(j)}$ и $x_{\sigma(j+1)}$ в цепи $G$ не лежит ни одной из вершин $x_{\sigma(1)}, \ldots, x_{\sigma(j-1)}, x_{\sigma(j+2)}, \ldots, x_{\sigma(i)}$ и для любого $j \in\{1, \ldots, i-2\}$ в цепи $G$ между вершинами $x_{\sigma(j)}$ и $x_{\sigma(j+2)}$ находится вершина $\left.x_{\sigma(j+1)}\right)$. Будем говорить, что вершины $x_{1}, \ldots, x_{i}, y_{1}, \ldots, y_{i}$ обладают свойством $i$-отделимости, если

- для любых $\nu_{1} \in\{1, \ldots, i-1\}, \nu_{2} \in\left\{\nu_{1}+1, \ldots, i\right\}$ выполнено

$$
d_{G}\left(x_{\nu_{1}}, x_{\nu_{2}}\right)<2^{k-i+1} \Leftrightarrow d_{H}\left(y_{\nu_{1}}, y_{\nu_{2}}\right)<2^{k-i+1}
$$

и, более того, если $d_{G}\left(x_{\nu_{1}}, x_{\nu_{2}}\right)<2^{k-i+1}$, то

$$
d_{G}\left(x_{\nu_{1}}, x_{\nu_{2}}\right)=d_{H}\left(y_{\nu_{1}}, y_{\nu_{2}}\right)
$$

- вершины $y_{\sigma\left(h_{1}\right)}, \ldots, y_{\sigma\left(h_{u}\right)}$ следуют подряд в цепи $H_{1}$, вершины $y_{\sigma\left(h_{u+1}\right)}, \ldots$, $y_{\sigma\left(h_{i}\right)}$ следуют подряд в цепи $H_{2}$, где $h_{1}<\cdots<h_{u}, h_{u+1}<\cdots<h_{i}$ - попарно различные числа, отображение $\sigma$ на множестве $\left\{h_{1}, \ldots, h_{u}\right\}$ принимает значения $j_{1}, \ldots, j_{u}$, а на множестве $\left\{h_{u+1}, \ldots, h_{i}\right\}$ - значения $j_{u+1}, \ldots, j_{i}$.

Сделанное нами предположение, очевидно, верно при $i=1$. Далее действуем по индукции и разбираем возможные случаи.

Пусть в $(i+1)$-м раунде Новатором выбрана вершина $x_{i+1}$ в графе $G$. Докажем, что Консерватор сможет выбрать такую вершину $y_{i+1} \in V(H)$, что вершины $x_{1}, \ldots, x_{i+1}, y_{1}, \ldots, y_{i+1}$ обладают свойством $(i+1)$-отделимости. 
Найдем "ближайшие" к вершине $x_{i+1}$ вершины среди $x_{1}, \ldots, x_{i}$. Если вершина $x_{i+1}$ в цепи $G$ лежит между вершинами $x_{\nu}$ и $x_{\mu}$ для некоторых $\nu, \mu \in$ $\{1, \ldots, i\}$, то Консерватор должен руководствоваться следующей стратегией. Если вершины $y_{\nu}$ и $y_{\mu}$ принадлежат одной цепи, то в силу свойства $i$-отделимости вершин $x_{1}, \ldots, x_{i}, y_{1}, \ldots, y_{i}$ Консерватор в графе $H$ сможет выбрать такую вершину $y_{i+1}$, что

$$
\begin{aligned}
& d_{G}\left(x_{\nu}, x_{i+1}\right)=d_{H}\left(y_{\nu}, y_{i+1}\right) I\left(d_{H}\left(y_{\nu}, y_{i+1}\right)<2^{k-i}\right)+d_{1} I\left(d_{H}\left(y_{\nu}, y_{i+1}\right) \geqslant 2^{k-i}\right), \\
& d_{G}\left(x_{\mu}, x_{i+1}\right)=d_{H}\left(y_{\mu}, y_{i+1}\right) I\left(d_{H}\left(y_{\mu}, y_{i+1}\right)<2^{k-i}\right)+d_{2} I\left(d_{H}\left(y_{\mu}, y_{i+1}\right) \geqslant 2^{k-i}\right)
\end{aligned}
$$

при некоторых $d_{1}, d_{2} \geqslant 2^{k-i}$. Если же вершины $y_{\nu}$ и $y_{\mu}$ принадлежат разным цепям, то хотя бы одно из чисел $d_{G}\left(x_{i+1}, x_{\nu}\right), d_{G}\left(x_{i+1}, x_{\mu}\right)$ не меньше, чем $2^{k-i}$. Будем для определенности считать, что $\sigma^{-1}(\nu)<\sigma^{-1}(\mu)$. И пусть, например, $d_{G}\left(x_{i+1}, x_{\nu}\right)<2^{k-i}$. Тогда Консерватор выберет такую вершину $y_{i+1}$, что $d_{H}\left(y_{i+1}, y_{\nu}\right)=d_{G}\left(x_{i+1}, x_{\nu}\right)$ и ближайшая к $y_{i+1}$ вершина среди $y_{1}, \ldots, y_{i}$ в $H$, не считая $y_{\nu}$, находится на расстоянии от нее, не меньшем $2^{k-i}$. Очевидно, что вершины $x_{1}, \ldots, x_{i+1}, y_{1}, \ldots, y_{i+1}$ обладают свойством $(i+1)$-отделимости. Если же $d_{G}\left(x_{i+1}, x_{\nu}\right) \geqslant 2^{k-i}, d_{G}\left(x_{i+1}, x_{\mu}\right) \geqslant 2^{k-i}$, то в силу свойства $i$-отделимости вершин $x_{1}, \ldots, x_{i}, y_{1}, \ldots, y_{i}$ выполнено неравенство $d_{H}\left(y_{\sigma^{-1}(\nu)}, y_{\sigma^{-1}(\nu+\chi)}\right) \geqslant 2^{k-i+1}$, где $\chi$ - наименьшее такое натуральное число, что вершина $y_{\sigma^{-1}(\nu+\chi)}$ принадлежит той же цепи, что и вершина $y_{\sigma^{-1}(\nu)}$, если такая вершина имеется. В этом случае (существует конечное число ұ) Консерватор сможет выбрать в той же цепи такую вершину $y_{i+1}$, что

$$
d_{H}\left(y_{\sigma^{-1}(\nu)}, y_{i+1}\right) \geqslant 2^{k-i}, \quad d_{H}\left(y_{\sigma^{-1}(\nu+\chi)}, y_{i+1}\right) \geqslant 2^{k-i}
$$

В противном случае Консерватор сможет выбрать такую вершину $y_{i+1}$, что

$$
\min _{j \in\{1, \ldots, i\}} d_{H}\left(y_{j}, y_{i+1}\right)=d_{H}\left(y_{\sigma^{-1}(\nu)}, y_{i+1}\right) \geqslant 2^{k-i} .
$$

Очевидно, что вершины $x_{1}, \ldots, x_{i+1}, y_{1}, \ldots, y_{i+1}$ обладают свойством $(i+1)$-отделимости.

Если, наконец, вершина $x_{i+1}$ в цепи $G$ является "крайней" и $i>1$, т. е. либо

$$
d_{G}\left(x_{i+1}, x_{\sigma(i-1)}\right)=d_{G}\left(x_{\sigma(i)}, x_{\sigma(i-1)}\right)+d_{G}\left(x_{i+1}, x_{\sigma(i)}\right),
$$

либо

$$
d_{G}\left(x_{i+1}, x_{\sigma(2)}\right)=d_{G}\left(x_{\sigma(1)}, x_{\sigma(2)}\right)+d_{G}\left(x_{i+1}, x_{\sigma(1)}\right),
$$

то Консерватор выберет такую новую "крайнюю вершину" $y_{i+1}$, принадлежащую цепи, которой принадлежит вершина $y_{\sigma(i)}$ (если выполнено равенство (4)) или вершина $y_{\sigma(1)}$ (если выполнено равенство (5)), что

$$
d_{H}\left(y_{\sigma(i)}, y_{i+1}\right)=d_{G}\left(x_{\sigma(i)}, x_{i+1}\right),
$$

если выполнено (4), и

$$
d_{H}\left(y_{\sigma(1)}, y_{i+1}\right)=d_{G}\left(x_{\sigma(1)}, x_{i+1}\right)
$$


если выполнено (5). Очевидно, что вершины $x_{1}, \ldots, x_{i+1}, y_{1}, \ldots, y_{i+1}$ и в этом случае обладают свойством $(i+1)$-отделимости. В случае $i=1$ стратегия Консерватора очевидна.

Пусть в $(i+1)$-м раунде Новатором выбрана вершина $y_{i+1}$ в графе $H_{1}$ (без ограничения общности выбираем одну из цепей графа $H$ ). Докажем, что Консерватор сможет выбрать такую вершину $x_{i+1} \in V(G)$, что вершины $x_{1}, \ldots, x_{i+1}, y_{1}, \ldots, y_{i+1}$ обладают свойством $(i+1)$-отделимости. Найдем "ближайшие" к вершине $y_{i+1}$ вершины среди $y_{1}, \ldots, y_{i}$ в цепи $H_{1}$. Пусть вершина $y_{i+1}$ в цепи $H_{1}$ лежит между вершинами $y_{\nu}$ и $y_{\mu}$ для некоторых $\nu, \mu \in\{1, \ldots, i\}$. Если $\left|\sigma^{-1}(\nu)-\sigma^{-1}(\mu)\right|=1$, то в силу свойства $i$-отделимости вершин $x_{1}, \ldots, x_{i}, y_{1}, \ldots, y_{i}$ Консерватор в графе $G$ сможет выбрать такую вершину $x_{i+1}$, что равенства $(1),(2)$ выполнены для некоторых $d_{1}, d_{2} \geqslant 2^{k-i}$. Если $\left|\sigma^{-1}(\nu)-\sigma^{-1}(\mu)\right|>1$, то в силу свойства $i$-отделимости вершин $x_{1}, \ldots, x_{i}$, $y_{1}, \ldots, y_{i}$ выполнены неравенства

$$
\begin{gathered}
d_{H_{1}}\left(y_{\nu}, y_{\mu}\right) \geqslant 2^{k-i+1}, \quad d_{G}\left(x_{\nu}, x_{\sigma\left(\sigma^{-1}(\nu)+1\right)}\right) \geqslant 2^{k-i+1}, \\
d_{G}\left(x_{\mu}, x_{\sigma\left(\sigma^{-1}(\mu)-1\right)}\right) \geqslant 2^{k-i+1}
\end{gathered}
$$

(для определенности будем считать, что $\left.\sigma^{-1}(\nu)<\sigma^{-1}(\mu)\right)$. Если, например, $d_{H_{1}}\left(y_{\nu}, y_{i+1}\right)<2^{k-i}$, то Консерватор сможет выбрать такую вершину $x_{i+1}$, что

$$
d_{G}\left(x_{\nu}, x_{i+1}\right)=d_{H_{1}}\left(y_{\nu}, y_{i+1}\right), \quad d_{G}\left(x_{i+1}, x_{\sigma\left(\sigma^{-1}(\nu)+1\right)}\right) \geqslant 2^{k-i} .
$$

Если, наконец, $\min \left\{d_{H_{1}}\left(y_{\nu}, y_{i+1}\right), d_{H_{1}}\left(y_{\mu}, y_{i+1}\right)\right\} \geqslant 2^{k-i}$, то Консерватор выберет такую вершину $x_{i+1}$, что $d_{G}\left(x_{\nu}, x_{i+1}\right)=2^{k-i}$. Легко заметить, что во всех рассмотренных случаях вершины $x_{1}, \ldots, x_{i+1}, y_{1}, \ldots, y_{i+1}$ обладают свойством $(i+1)$-отделимости. Случай "крайней” вершины $y_{i+1}$ разбирается аналогичным образом.

Таким образом, если Консерватор будет руководствоваться описанной стратегией, то по прошествии $k$ раундов выбранные вершины $x_{1}, \ldots, x_{k}, y_{1}, \ldots, y_{k}$ будут обладать свойством $k$-отделимости и, следовательно, индуцированные подграфы $\left.G\right|_{\left\{x_{1}, \ldots, x_{k}\right\}}$ и $\left.H\right|_{\left\{y_{1}, \ldots, y_{k}\right\}}$ будут изоморфны. Поэтому Консерватор победит. Тем самым, мы доказали, что свойство связности нельзя выразить формулой первого порядка, а стало быть, формулами первого порядка не ограничиваются формальные записи всех возможных свойств. Свойство связности графа выразимо формулой второго порядка, т. е. формулой, в которой кванторы ставятся и по предикатам:

$(\exists X \forall x \exists y[[(X(x)) \Rightarrow((\neg(X(y))) \wedge(x \sim y))] \wedge[(\neg(X(x))) \Rightarrow((X(y)) \wedge(x \sim y))]])$.

Это свойство означает, что множество вершин графа можно так разбить на два подмножества $X$ и $\bar{X}$, что для любой вершины из $X$ найдется вершина из $\bar{X}$, соединенная с ней ребром, и для любой вершины из $\bar{X}$ найдется вершина из $X$, соединенная с ней ребром (иными словами, граф связен). 


\section{4. Закон нуля или единицы для $G(N, p)$ при $p=$ const}

Определение закона нуля или единицы для случайного графа $\mathscr{G}\left(G_{n}, p\right)$ для класса свойств $\mathscr{C}$ было дано в разделе 2 . Если $\mathscr{C}$ - класс всех свойств первого порядка, то мы будем просто говорить, что случайный граф $\mathscr{G}\left(G_{n}, p\right)$ подчиняется закону нуля или единицы. Такое сокращение мотивировано тем, что, как мы покажем в этом разделе, случайный граф $G(N, p)$, где $p$ - константа, подчиняется закону нуля или единицы для класса свойств первого порядка и этот результат нельзя расширить до языка второго порядка.

Последнее обстоятельство совсем легко прояснить. Докажем, что, например, граф $G(N, 0.5)$ не подчиняется закону нуля или единицы для класса свойств второго порядка. Рассмотрим формулу второго порядка

$$
\begin{gathered}
\left(\exists X \forall x _ { 1 } \forall y _ { 1 } \exists x _ { 2 } \exists y _ { 2 } \left(( x _ { 1 } \sim y _ { 1 } ) \Rightarrow \left(\left(X\left(x_{1}, y_{1}, x_{2}, y_{2}\right)\right) \wedge\left(x_{2} \nsim y_{2}\right)\right.\right.\right. \\
\wedge\left(\forall x \forall y\left(\left(\left(x \neq x_{2}\right) \vee\left(y \neq y_{2}\right)\right) \Rightarrow\left(\neg\left(X\left(x_{1}, y_{1}, x, y\right)\right)\right)\right)\right) \\
\left.\left.\wedge\left(\forall x \forall y\left(\left(\left(x \neq x_{1}\right) \vee\left(y \neq y_{1}\right)\right) \Rightarrow\left(\neg\left(X\left(x, y, x_{2}, y_{2}\right)\right)\right)\right)\right)\right)\right) .
\end{gathered}
$$

Свойство, выраженное этой формулой, означает, что не более половины пар вершин в графе образует ребра. Вероятность того, что в графе $G(N, 0.5)$ количество ребер не превосходит $N(N-1) / 4$, равна

$$
\sum_{i=0}^{[N(N-1) / 4]} C_{N(N-1) / 2}^{i}\left(\frac{1}{2}\right)^{N(N-1) / 2} \rightarrow \frac{1}{2}, \quad N \rightarrow \infty .
$$

Таким образом, предел отличен от 0 и от 1 , а следовательно, закон нуля или единицы для класса свойств второго порядка не выполнен.

Перейдем теперь к обсуждению справедливости закона нуля или единицы для $G(N, p)$ с постоянным $p$ и класса свойств первого порядка. Дадим некий удобный критерий, верный при любых $p$, не только постоянных. Этот критерий будет следствием из теоремы 6 (см., например, [8]). Итак, обозначим $\mathrm{P}_{N, M, p}$ декартово произведение мер $\mathrm{P}_{N, p} \times \mathrm{P}_{M, p}$. Тогда имеет место следующая теорема.

ТЕОрема 7. Случайный граф $G(N, p)$ подчиняется закону нуля или единииъь тогда и толъко тогда, когда для любого $k \in \mathbb{N}$

$$
\begin{aligned}
\lim _{N, M \rightarrow \infty} \mathrm{P}_{N, M, p}(\{(A, B): & \text { y Консерватора есть выигрышная } \\
& \text { стратегия в игре } \operatorname{EHR}(A, B, k)\})=1 .
\end{aligned}
$$

ДокАЗАТЕльство. Пусть выполнен закон нуля или единицы. Предположим, что при некотором $k \in \mathbb{N}$ предел вероятности существования выигрышной стратегии Консерватора в игре с $k$ раундами либо не существует, либо не равен 1. Тогда существует частичный предел, отличный от 1. Иными словами, найдутся такие возрастающие последовательности чисел $N_{i} \uparrow \infty, M_{i} \uparrow \infty$, что предел вероятности существования выигрышной стратегии Консерватора в игpe $\operatorname{EHR}\left(G\left(N_{i}, p\left(N_{i}\right)\right), G\left(M_{i}, p\left(M_{i}\right)\right), k\right)$ равен $c$, где $0 \leqslant c<1$. Пусть $X_{N, M}-$ множество всех таких пар остовных подграфов $A, B$ в полных графах $K_{N}, K_{M}$ соответственно, что у Новатора есть выигрышная стратегия в игре $\operatorname{EHR}(A, B, k)$. 
Тогда, очевидно,

$$
\lim _{i \rightarrow \infty} \mathrm{P}_{N_{i}, M_{i}, p}\left(X_{N_{i}, M_{i}}\right)=1-c .
$$

В силу теоремы 6 для любой пары $(A, B) \in X_{N, M}$ существует такое свойство первого порядка $L(A, B)$, кванторная глубина которого ограничена числом $k$, что либо $A \in L(A, B), B \notin L(A, B)$, либо $A \notin L(A, B), B \in L(A, B)$.

Заметим, что существует лишь конечное количество различных свойств, выражаемых формулами кванторной глубины $k$ (см., например, [46]). Для любых $N, M \in \mathbb{N}$ обозначим $\mathscr{U}(N, M)$ множество всех различных свойств среди $L(A, B),(A, B) \in X_{N, M}$. Пусть $\mathscr{U}$ - множество всех различных свойств в $\bigcup_{i \in \mathbb{N}} \mathscr{U}\left(N_{i}, M_{i}\right)$. Имеем

$$
\begin{aligned}
\mathrm{P}_{N_{i}, M_{i}, p}\left(X_{N_{i}, M_{i}}\right) & =\mathrm{P}_{N_{i}, M_{i}, p}\left(\bigcup_{L \in \mathscr{U}}\left(\left(L^{N_{i}} \times \overline{L^{M_{i}}}\right) \cup\left(\overline{L^{N_{i}}} \times L^{M_{i}}\right)\right)\right) \\
& \leqslant \sum_{L \in \mathscr{U}} \mathrm{P}_{N_{i}, M_{i}, p}\left(\left(L^{N_{i}} \times \overline{L^{M_{i}}}\right) \cup\left(\overline{L^{N_{i}}} \times L^{M_{i}}\right)\right) .
\end{aligned}
$$

Пусть $\delta$ - такое положительное число, что $c+\delta<1$. Тогда существует такое $i_{0} \in \mathbb{N}$, что для всех $i>i_{0}$ справедливо неравенство

$$
\mathrm{P}_{N_{i}, M_{i}, p}\left(X_{N_{i}, M_{i}}\right)>1-c-\delta .
$$

Следовательно, для каждого $i>i_{0}$ существует такое $L=L(i) \in \mathscr{U}$, что

$$
\mathrm{P}_{N_{i}, M_{i}, p}\left(\left(L^{N_{i}} \times \overline{L^{M_{i}}}\right) \cup\left(\overline{L^{N_{i}}} \times L^{M_{i}}\right)\right)>\frac{1-c-\delta}{|\mathscr{U}|} .
$$

Поэтому существуют такие последовательность $\left\{i_{j}\right\}_{j \in \mathbb{N}}$ и свойство $L \in \mathscr{U}$, что для любого $j \in \mathbb{N}$

$$
\mathrm{P}_{N_{i_{j}}, M_{i_{j}}, p}\left(\left(L^{N_{i_{j}}} \times \overline{L^{M_{i_{j}}}}\right) \cup\left(\overline{L^{N_{i_{j}}}} \times L^{M_{i_{j}}}\right)\right)>\frac{1-c-\delta}{|\mathscr{U}|} .
$$

Значит,

$$
\max \left\{\mathrm{P}_{N_{i_{j}}, M_{i_{j}}, p}\left(L^{N_{i_{j}}} \times \overline{L^{M_{i_{j}}}}\right), \mathrm{P}_{N_{i_{j}}, M_{i_{j}}, p}\left(\overline{L^{N_{i_{j}}}} \times L^{M_{i_{j}}}\right)\right\}>\frac{1-c-\delta}{2|\mathscr{U}|} .
$$

Так как $L$ - свойство первого порядка, выражаемое формулой с ограниченной числом $k$ кванторной глубиной, то $\lim _{N \rightarrow \infty} \mathrm{P}_{N, p}(L) \in\{0,1\}$. А стало быть, существуют сколь угодно большие $N, M$, при которых

$$
\mathrm{P}_{N, M, p}\left(L^{N} \times \overline{L^{M}}\right)>\frac{1-c-\delta}{2|\mathscr{U}|} .
$$

Получили противоречие.

Зафиксируем теперь произвольное $k \in \mathbb{N}$ и предположим, что предел вероятности того, что у Консерватора есть выигрышная стратегия в игре с $k$ раундами, равен 1. Докажем, что утверждение теоремы верно для любой формулы, кванторная глубина которой ограничена числом $k$. Предположим, что 
найдется такая формула первого порядка глубины $k$, что либо предела вероятности обладания соответствующим свойством $L$ не существует и все частичные пределы принадлежат множеству $\{0,1\}$, либо существует (частичный) предел, отличный от нуля и единицы.

В первом случае пусть $N_{i} \uparrow \infty, M_{i} \uparrow \infty$ - такие последовательности, что $\lim _{i \rightarrow \infty} \mathrm{P}_{N_{i}, p}(L)=0, \lim _{i \rightarrow \infty} \mathrm{P}_{M_{i}, p}(L)=1$. Тогда

$$
\lim _{i \rightarrow \infty} \mathrm{P}_{N_{i}, M_{i}, p}\left(\overline{L^{N_{i}}} \times L^{M_{i}}\right)=1 .
$$

В силу теоремы 6 для любого $i \in \mathbb{N}$ и любой пары $(A, B) \in \overline{L^{N_{i}}} \times L^{M_{i}}$ у Новатора есть выигрышная стратегия в игре $\operatorname{EHR}(A, B, k)$. Следовательно,

$$
\begin{aligned}
\lim _{i \rightarrow \infty} \mathrm{P}_{N_{i}, M_{i}, p}(\{(A, B): & \text { у Новатора есть выигрышная } \\
& \text { стратегия в игре } \operatorname{EHR}(A, B, k)\})=1 .
\end{aligned}
$$

Получили противоречие.

Во втором случае существует частичный предел, отличный от 0 и 1 . Пусть $N_{i} \uparrow \infty$ - такая последовательность, что $\lim _{i \rightarrow \infty} \mathrm{P}_{N_{i}, p}(L)=c \in(0,1)$. Тогда

$$
\lim _{i \rightarrow \infty} \mathrm{P}_{N_{i}, N_{i+1}, p}\left(\overline{L^{N_{i}}} \times L^{N_{i+1}}\right)=(1-c) c .
$$

В силу теоремы 6 для любого $i \in \mathbb{N}$ и любой пары $(A, B) \in \overline{L^{N_{i}}} \times L^{N_{i+1}}$ у Новатора есть выигрышная стратегия в игре $\operatorname{EHR}(A, B, k)$. Следовательно, при достаточно больших $i$ имеем

$$
\begin{aligned}
\lim _{i \rightarrow \infty} \mathrm{P}_{N_{i}, N_{i+1}, p}(\{(A, B): & \text { у Новатора есть выигрышная } \\
& \text { стратегия в игре } \operatorname{EHR}(A, B, k)\})>\frac{(1-c) c}{2} .
\end{aligned}
$$

Снова получили противоречие. Теорема доказана.

В 1969 г. Ю. В. Глебский, Д. И. Коган, М. И. Лиогонький и В. А. Таланов (и независимо в 1976 г. Р. Фагин) доказали, что случайный граф $G(N, p)$ подчиняется закону нуля или единицы, если $p$ не зависит от $N$.

Теорема 8 (Ю. В. Глебский, Д. И. Коган, М. И. Лиогонький и В. А. Таланов, 1969, [51]; Р. Фагин, 1976, [52]). Случайнъй граф $G(N, p)$ при фиксированном р подчиняется закону нуля или единищъ.

ДоказАтельство. В случае $p \in\{0,1\}$ утверждение теоремы очевидно. Пусть $p \in(0,1)$. В силу теоремы 7 для доказательства теоремы достаточно предъявить стратегию Консерватора, которая является выигрышной с вероятностью, стремящейся к 1. Эта стратегия опирается на свойство графов, определенное ниже, которое мы будем использовать и в следующих разделах. Пусть $s \in \mathbb{N}$. Будем говорить, что граф $H$ обладает свойством полного расширения уровня $s$ (которое мы будем обозначать $S_{s}$ ), если для любых целых неотрицательных чисел $a, b$, удовлетворяющих неравенству $a+b \leqslant s$, и для любых вершин $v_{1}, \ldots, v_{a}, u_{1}, \ldots, u_{b} \in V(H)$ найдется такая вершина $z \in V(H)$, что для любых 
$i \in\{1, \ldots, a\}, j \in\{1, \ldots, b\}$ вершины $v_{i}, z$ соединены ребром в $H$, а вершины $u_{j}, z$ не соединены ребром в $H$. Легко заметить, что если графы $A, B$ обладают свойством полного расширения уровня $s$, то у Консерватора есть выигрышная стратегия в игре $\operatorname{EHR}(A, B, s)$. Поэтому для доказательства теоремы достаточно убедиться в том, что для любого $s \in \mathbb{N}$ случайный граф $G(N, p)$ обладает свойством $S_{s}$ с вероятностью, стремящейся к 1 . Пусть $v_{1}, \ldots, v_{a}$, $u_{1}, \ldots, u_{b} \in V_{N}$ для некоторых чисел $a$ и $b$. Обозначим $U_{v_{1}, \ldots, v_{a}}^{u_{1}, \ldots, u_{b}}$ множество всех таких графов из $\Omega_{N}$, что существует вершина $z \in V_{N}$, соединенная ребром с каждой вершиной из $v_{1}, \ldots, v_{a}$ и не соединенная ни с одной из $u_{1}, \ldots, u_{b}$. Заметим, что

$$
\mathrm{P}_{N, p}\left(\overline{U_{v_{1}, \ldots, v_{a}}^{u_{1}, \ldots, u_{b}}}\right)=\left(1-p^{a}(1-p)^{b}\right)^{N-a-b} \leqslant\left(1-\min \{p, 1-p\}^{s}\right)^{N-s} .
$$

Поэтому

$$
\begin{aligned}
\mathrm{P}_{N, p}\left(\overline{S_{s}}\right) & =\mathrm{P}_{N, p}\left(\bigcup_{a=0}^{s} \bigcup_{b=0}^{s-a} \bigcup_{v_{1}, \ldots, v_{a}, u_{1}, \ldots, u_{b} \in V_{N}} \overline{U_{v_{1}, \ldots, v_{a}}^{u_{1}, \ldots, u_{b}}}\right) \\
& \leqslant s^{2} N^{s}\left(1-\min \{p, 1-p\}^{s}\right)^{N-s} \rightarrow 0, \quad N \rightarrow \infty .
\end{aligned}
$$

Теорема доказана.

В следующем разделе мы сформулируем и докажем законы нуля или единицы для случайного графа $G(N, p)$, где $p$ - различные функции от количества вершин $N$.

5. Законы нуля или единицы для $G(N, p)$ при $p \neq$ const

Доказательство теоремы 8 не изменится, если в рассуждениях заменить константу $p$ на функцию $p=p(N)$, которая при каждом $\alpha>0$ обладает свойством (1). Поэтому для таких функций $p$ также справедлив закон нуля или единицы.

Теорема 9. Пусть $p: \mathbb{N} \rightarrow[0,1]$ - функиия, которая при каждом $\alpha>0$ обладает свойством (1). Тогда случайный граф $G(N, p)$ подчиняется закону нуля или единицы.

В разделе 2 мы доказали, что из существования для любого рационального $\alpha \in(0,1]$ строго сбалансированного графа с плотностью $1 / \alpha$ следует отсутствие закона нуля или единицы для случайного графа $G\left(N, N^{-\alpha}\right)$. Более того, справедлив следующий результат.

Теорема 10 (Дж. Спенсер, С. Шела, 1988, [50]; А. Ручински, А. Винс, 1986, [20]). Пусть $\alpha>0$ - рачиональное число. Если $\alpha>2$ или $\alpha \in(1+$ $1 /(l+1), 1+1 / l)$ для некоторого $l \in \mathbb{N}$, то случайный граф $G\left(N, N^{-\alpha}\right)$ подчиняется закону нуля или единицы. Во всех остальных случаях случайный граф $G\left(N, N^{-\alpha}\right)$ закону нуля или единицы не подчиняется. 
ДокАЗАтельство. Разобьем рассуждение на три части. В первой части мы дадим набросок доказательства того, что при $\alpha \in(0,1]$ закона нуля или единицы нет. Во второй части мы обсудим отсутствие этого закона при $\alpha=$ $1+1 / l, l \in \mathbb{N}$, и при $\alpha>2$. А третью часть посвятим доказательству этого закона при $\alpha \in(1+1 /(l+1), 1+1 / l)$.

СлучАй 1: $\alpha \in(0,1]$. Как уже было сказано выше, случай $\alpha \in(0,1]$ следует из теоремы 4 и существования строго сбалансированного графа с плотностью $1 / \alpha$, которое доказано в [20]. В настоящей работе мы не приводим ни доказательства первого факта, ни полного доказательства последнего факта. Однако последний факт мы все же частично обоснуем. А именно, мы предполагаем, что утверждение о существовании строго сбалансированного графа доказано для $\alpha \in(2 / 3,1]$, и затем для любого $\alpha \leqslant 2 / 3$ приводим конструкцию строго сбалансированного графа с плотностью $1 / \alpha$. Итак, во-первых, справедлива следующая лемма.

Лемма 1. Пусть $G$ - строго сбалансированный граф с плотностью $\rho$. Тогда существует строго сбалансированный граф с плотностью $1 / 2+\rho$.

ДоказАтельство. Пусть $G_{1}, G_{2}$ - графы, изоморфные $G$ и не имеющие общих вершин. Пусть, кроме того, $V\left(G_{1}\right)=\left\{x_{1}^{1}, \ldots, x_{n}^{1}\right\}, V\left(G_{2}\right)=\left\{x_{1}^{2}, \ldots, x_{n}^{2}\right\}$, причем существует такой изоморфизм $f: G_{1} \rightarrow G_{2}$, что $f\left(x_{i}^{1}\right)=x_{i}^{2}$ для любого $i \in\{1, \ldots, n\}$. Определим граф $H$ следующим образом:

$$
V(H)=V\left(G_{1}\right) \cup V\left(G_{2}\right), \quad E(H)=E\left(G_{1}\right) \cup E\left(G_{2}\right) \cup\left\{\left\{x_{1}^{1}, x_{1}^{2}\right\}, \ldots,\left\{x_{n}^{1}, x_{n}^{2}\right\}\right\} .
$$

Очевидно, что плотность графа $H$ равна $1 / 2+\rho$. Докажем, что он строго сбалансированный. Пусть $K-$ некоторый подграф графа $H$. Положим $K \cap$ $G_{1}=K_{1}, K \cap G_{2}=K_{2}$. Обозначим $e=e(K)-e\left(K_{1}\right)-e\left(K_{2}\right)$. Так как $G-$ строго сбалансированный граф и $e \leqslant \min \left\{v\left(K_{1}\right), v\left(K_{2}\right)\right\}$, имеем

$$
\begin{aligned}
\rho(K) & =\frac{e\left(K_{1}\right)+e\left(K_{2}\right)+e}{v\left(K_{1}\right)+v\left(K_{2}\right)}<\frac{v\left(K_{1}\right) \rho+v\left(K_{2}\right) \rho+e}{v\left(K_{1}\right)+v\left(K_{2}\right)} \\
& =\rho+\frac{e}{v\left(K_{1}\right)+v\left(K_{2}\right)} \leqslant \rho+\frac{1}{2}=\rho(H) .
\end{aligned}
$$

Лемма доказана.

Теперь предположим, что для любого $\alpha \in(2 / 3,1]$ существование строго сбалансированного графа с плотностью $1 / \alpha$ доказано. Докажем, что существует строго сбалансированный граф с плотностью $\rho$, где $\rho \geqslant 3 / 2$ - произвольное число. Число $\rho$ допускает единственное представление в виде

$$
\rho=\frac{1}{2} n+\rho_{0}, \quad \text { где } \quad n \in \mathbb{N}, \quad \rho_{0} \in\left[1, \frac{3}{2}\right) .
$$

Так как строго сбалансированный граф с плотностью $\rho_{0}$ существует, то по лемме 1 существует и строго сбалансированный граф с плотностью $\rho$.

В случае 1 доказательство теоремы завершено. 
СлучАй $2: \alpha=1+1 / l, l \in \mathbb{N}$, или $\alpha>2$. Отсутствие закона нуля или единицы при $\alpha=1+1 / l$, где $l \in \mathbb{N}$, следует из того, что для любого $l \in \mathbb{N}$ существует дерево (которое, очевидно, является строго сбалансированным графом) с плотностью $l /(l+1)$. Если $\alpha>2$, то с вероятностью $\left(1-N^{-\alpha}\right)_{N}^{2}$, стремящейся к 1 , в графе $G\left(N, N^{-\alpha}\right)$ нет ребер, а следовательно, случайный граф подчиняется закону нуля или единицы.

В случае 2 доказательство теоремы завершено.

СлУчАЙ 3: $\alpha \in(1+1 /(l+1), 1+1 / l)$. Пусть, наконец, $l \in \mathbb{N}$ и $\alpha \in$ $(1+1 /(l+1), 1+1 / l)$. Пусть, кроме того, $k \in \mathbb{N}$ - произвольное число. Для каждого $N \in \mathbb{N}$ обозначим $\widetilde{\Omega}_{N}$ множество всех графов в $\Omega_{N}$, обладающих следующим свойством. Граф $G$ принадлежит $\widetilde{\Omega}_{N}$ тогда и только тогда, когда любой его подграф на $l+2$ вершинах не является связным, любой его связный подграф является деревом, для любого дерева $H$ на не более чем $l+1$ вершинах в $G$ существует не менее $k$ компонент, являющихся копиями $H$. Из теоремы 2 следует, что с вероятностью, стремящейся к 1 , в случайном графе $G\left(N, N^{-\alpha}\right)$ любой подграф на $l+2$ вершинах не является связным, любой связный подграф является деревом, существует копия любого дерева $H$ на не более чем $l+1$ вершинах. Докажем, что более сильное утверждение $\lim _{N \rightarrow \infty} \mathrm{P}_{N, N^{-\alpha}}\left(\widetilde{\Omega}_{N}\right)=1$ следует из сформулированного ниже результата. Обозначим $\widetilde{N}_{G}$ случайную величину, равную наибольшему количеству копий графа $G$ в случайном графе $G(N, p)$, не пересекающихся по вершинам.

Теорема 11 (Б. Кройтер, 1996, [53]). Пусть $p: \mathbb{N} \rightarrow[0,1]$ - произвольная функиия, $G$ - произвольный граф. Обозначим

$$
\Phi_{G}(N)=\min _{\varnothing \subset H \subseteq G}\left\{N^{v(H)} p^{e(H)}\right\} .
$$

Если $\Phi_{G}(N) \rightarrow \infty$ при $N \rightarrow \infty$, то для некоторьх чисел $c, C>0$

$$
\mathrm{P}_{N, p}\left(c \Phi_{G}(N) \leqslant \widetilde{N}_{G} \leqslant N_{G} \leqslant C \Phi_{G}(N)\right) \rightarrow 1 .
$$

Пусть $G_{1} \subset G_{2}$ - два дерева, $v\left(G_{2}\right) \leqslant l+1$. Тогда, очевидно,

$$
\Phi_{G_{1}}(N)=N^{v\left(G_{1}\right)-\alpha\left(v\left(G_{1}\right)-1\right)}=N^{\alpha-(\alpha-1) v\left(G_{1}\right)}>N^{\alpha-(\alpha-1) v\left(G_{2}\right)}=\Phi_{G_{2}}(N) .
$$

Поэтому в силу теоремы 11 с вероятностью, стремящейся к 1 , количество компонент, являющихся копиями $G_{1}$, в случайном графе $G\left(N, N^{-\alpha}\right)$ стремится к бесконечности. Следовательно, действительно, $\lim _{N \rightarrow \infty} \mathrm{P}_{N, N^{-\alpha}}\left(\widetilde{\Omega}_{N}\right)=1$.

Пусть $N, M \in \mathbb{N}, A \in \widetilde{\Omega}_{N}, B \in \widetilde{\Omega}_{M}$. Докажем, что в этом случае у Консерватора есть выигрышная стратегия в игре $\operatorname{EHR}(A, B, k)$ (а стало быть, по доказанному вероятность того, что у Консерватора есть выигрышная стратегия в игре $\operatorname{EHR}(G(N, p(N)), G(M, p(M)), k)$, стремится к 1 при $N, M \rightarrow \infty)$. В первом раунде Новатор выбирает некоторую вершину $x_{1}$ в одном из графов (например, в $A$ ). Пусть $X_{1}$ - древесная компонента в $A$, содержащая $x_{1}$. Тогда, так как $B \in \widetilde{\Omega}_{M}$, в $B$ найдется компонента $Y_{1}$, изоморфная $X_{1}$. Пусть при соответствующем изоморфизме вершина $x_{1}$ переходит в вершину $y_{1}$. Эту вершину и выберет Консерватор в первом раунде. Пусть сыграно $i$ раундов, 
$1 \leqslant i<k$. Пусть, кроме того, в графе $A$ выбраны вершины $x_{1}, \ldots, x_{i}$ и компоненты $X_{1}, \ldots, X_{j}, j \leqslant i$, в графе $B$ выбраны вершины $y_{1}, \ldots, y_{i}$ и компоненты $Y_{1}, \ldots, Y_{j}$ и при этом выполнены следующие свойства.

(i.1) Существует изоморфизм $f: X_{1} \cup \cdots \cup X_{j} \rightarrow Y_{1} \cup \cdots \cup Y_{j}$, переводящий граф $X_{r}$ в граф $Y_{r}$ для каждого $r \in\{1, \ldots, j\}$.

$(i .2)$ Вершины $x_{1}, \ldots, x_{i}$ принадлежат графу $X_{1} \cup \cdots \cup X_{j}$, вершины $y_{1}, \ldots, y_{i}$ принадлежат графу $Y_{1} \cup \cdots \cup Y_{j}$.

Пусть в $(i+1)$-м раунде Новатор выбирает вершину $y_{i+1}$, например, в графе $B$. Если $y_{i+1} \in V\left(Y_{1} \cup \cdots \cup Y_{j}\right)$, то Консерватор выберет вершину $x_{i+1}=$ $f^{-1}\left(y_{i+1}\right)$. Таким образом, выбранные в $(i+1)$-м раунде в графе $A$ вершины $x_{1}, \ldots, x_{i+1}$ и компоненты $X_{1}, \ldots, X_{j}$ и выбранные тогда же в графе $B$ вершины $y_{1}, \ldots, y_{i+1}$ и компоненты $Y_{1}, \ldots, Y_{j}$ обладают свойствами $(i+1.1)$ и $(i+1.2)$. Если же $y_{i+1} \notin V\left(Y_{1} \cup \cdots \cup Y_{j}\right)$, то найдем компоненту $Y_{j+1}$ в графе $B$, содержащую вершину $y_{i+1}$. Так как $A \in \widetilde{\Omega}_{N}$, то в $A$ найдется компонента $X_{j+1}$, изоморфная $Y_{j+1}$. Пусть при соответствующем изоморфизме вершина $y_{j+1}$ переходит в вершину $x_{j+1}$. Эту вершину и выберет Консерватор в $(i+1)$-м раунде. Таким образом, и в этом случае выбранные в $(i+1)$-м раунде в графе $A$ вершины $x_{1}, \ldots, x_{i+1}$ и компоненты $X_{1}, \ldots, X_{j+1}$ и выбранные тогда же в графе $B$ вершины $y_{1}, \ldots, y_{i+1}$ и компоненты $Y_{1}, \ldots, Y_{j+1}$ обладают свойствами $(i+1.1)$ и $(i+1.2)$. Поэтому в последнем раунде Консерватор одержит победу, ведь компоненты, содержащие выбранные обоими игроками вершины, окажутся изоморфными. В силу теоремы 7 случайный граф $G\left(N, N^{-\alpha}\right)$ подчиняется закону нуля или единицы.

Рассмотрение случая 3 завершено, и теорема 10 доказана.

В 1988 г. в той же работе [50] Дж. Спенсер и С. Шела доказали, что случайный граф $G\left(N, N^{-\alpha}\right)$ подчиняется закону нуля или единицы при любом иррациональном $\alpha>0$. Прежде чем сформулировать и доказать теорему, проведем построение необходимых для этого конструкций.

Рассмотрим такие графы $H, G, \widetilde{H}, \widetilde{G}$, что

$$
\begin{array}{ll}
V(H)=\left\{x_{1}, \ldots, x_{k}\right\}, & V(G)=\left\{x_{1}, \ldots, x_{l}\right\}, \\
V(\widetilde{H})=\left\{\widetilde{x}_{1}, \ldots, \widetilde{x}_{k}\right\}, & V(\widetilde{G})=\left\{\widetilde{x}_{1}, \ldots, \widetilde{x}_{l}\right\},
\end{array}
$$

причем $H \subset G, \widetilde{H} \subset \widetilde{G}$ (тем самым, $k<l$ ). Граф $\widetilde{G}$ называется $(G, H)$-расширением графа $\widetilde{H}$, когда

$$
\left\{x_{i_{1}}, x_{i_{2}}\right\} \in E(G) \backslash E(H) \Rightarrow\left\{\widetilde{x}_{i_{1}}, \widetilde{x}_{i_{2}}\right\} \in E(\widetilde{G}) \backslash E(\widetilde{H}) .
$$

Если выполняется соотношение

$$
\left\{x_{i_{1}}, x_{i_{1}}\right\} \in E(G) \backslash E(H) \Leftrightarrow\left\{\widetilde{x}_{i_{1}}, \widetilde{x}_{i_{2}}\right\} \in E(\widetilde{G}) \backslash E(\widetilde{H}),
$$

то $\widetilde{G}$ называется точным расширением, а пары $(G, H)$ и $(\widetilde{G}, \widetilde{H})$ считаются изоморфными. Зафиксируем число $\alpha>0$. Положим

$$
\begin{gathered}
v(G, H)=|V(G) \backslash V(H)|, \quad e(G, H)=|E(G) \backslash E(H)|, \\
f_{\alpha}(G, H)=v(G, H)-\alpha e(G, H) .
\end{gathered}
$$


Если для любого такого графа $S$, что $H \subset S \subseteq G$, выполнено неравенство $f_{\alpha}(S, H)>0$, то пара $(G, H)$ называется $\alpha$-надежсной (см. [8], [10]). Если же для любого такого $S$, что $H \subseteq S \subset G$, выполнено неравенство $f_{\alpha}(G, S)<0$, то пара $(G, H)$ называется $\alpha$-жесткой. Введем, наконец, понятие максимальной пары. Пусть $\widetilde{H} \subset \widetilde{G} \subset \Gamma$ и $T \subset K$, причем $|V(T)| \leqslant|V(\widetilde{G})|$. Пару $(\widetilde{G}, \widetilde{H})$ назовем $(K, T)$-максималъной в $\Gamma$, если у любого такого подграфа $\widetilde{T}$ графа $\widetilde{G}$, что $|V(\widetilde{T})|=|V(T)|$ и $\widetilde{T} \cap \widetilde{H} \neq \widetilde{T}$, не существует такого точного $(K, T)$-расширения $\widetilde{K}$ в $\Gamma \backslash(\widetilde{G} \backslash \widetilde{T})$, что каждая вершина из $V(\widetilde{K}) \backslash V(\widetilde{T})$ не соединена ребром ни с одной вершиной из $V(\widetilde{G}) \backslash V(\widetilde{T})$. Граф $\widetilde{G}$ называется $(K, T)$-максимальным в $\Gamma$, если у любого такого подграфа $\widetilde{T}$ графа $\widetilde{G}$, что $|V(\widetilde{T})|=|V(T)|$, не существует такого точного $(K, T)$-расширения $\widetilde{K}$ в $\Gamma \backslash(\widetilde{G} \backslash \widetilde{T})$, что каждая вершина из $V(\widetilde{K}) \backslash V(\widetilde{T})$ не соединена ребром ни с одной вершиной из $V(\widetilde{G}) \backslash V(\widetilde{T})$.

Обратимся теперь к случайному графу $G(N, p)$. Пусть $\alpha>0, p=N^{-\alpha}$. Пусть, кроме того, пара $(G, H)$ является $\alpha$-надежной и

$$
V(H)=\left\{x_{1}, \ldots, x_{k}\right\}, \quad V(G)=\left\{x_{1}, \ldots, x_{l}\right\} .
$$

Рассмотрим произвольные вершины $\widetilde{x}_{1}, \ldots, \widetilde{x}_{k} \in V_{N}$ и случайную величину $N_{(G, H)}\left(\widetilde{x}_{1}, \ldots, \widetilde{x}_{k}\right)$ на вероятностном пространстве $\left(\Omega_{N}, \mathscr{F}_{N}, \mathrm{P}_{N, p}\right)$, которая каждому графу $\mathscr{G}$ из $\Omega_{N}$ ставит в соответствие количество $(G, H)$-расширений подграфа в $\mathscr{G}$, индуцированного на $\left\{\widetilde{x}_{1}, \ldots, \widetilde{x}_{k}\right\}$ (граф $X$ является подграфом графа $Y$, индуиированным на множество $S \subset V(Y)$, если $V(X)=S$ и для любых вершин $x, y \in S$ справедливо $\{x, y\} \in E(X) \Leftrightarrow\{x, y\} \in E(Y))$. Иными словами, пусть $W \subset V_{N} \backslash\left\{\widetilde{x}_{1}, \ldots, \widetilde{x}_{k}\right\}$ - множество, мощность $|W|$ которого равна $l-k$. Если можно так занумеровать элементы множества $W$ числами $k+1, k+2, \ldots, l$, что граф $\left.\mathscr{G}\right|_{\left\{\widetilde{x}_{1}, \ldots, \widetilde{x}_{l}\right\}}$ является $(G, H)$-расширением графа $\left.\mathscr{G}\right|_{\left\{\widetilde{x}_{1}, \ldots, \widetilde{x}_{k}\right\}}$, то положим $I_{W}(\mathscr{G})=1$. В противном случае $I_{W}(\mathscr{G})=0$. Случайная величина $N_{(G, H)}\left(\widetilde{x}_{1}, \ldots, \widetilde{x}_{k}\right)$ определяется следующим равенством:

$$
N_{(G, H)}\left(\widetilde{x}_{1}, \ldots, \widetilde{x}_{k}\right)=\sum_{W \subset V_{N} \backslash\left\{\widetilde{x}_{1}, \ldots, \widetilde{x}_{k}\right\},|W|=l-k} I_{W}
$$

Обозначим $\mathrm{E}_{N, p}$ математическое ожидание по мере $\mathrm{P}_{N, p}$.

Теорема 12 (Дж. Спенсер, 1990, [54]). С вероятностъю, стремящейся $\kappa 1$, для любых вершин $\widetilde{x}_{1}, \ldots, \widetilde{x}_{k}$ и для любого $\varepsilon>0$ найдется такое $N_{0}$, что для любого натурального $N>N_{0}$ справедливо соотношение

$$
\begin{aligned}
(1-\varepsilon) \mathrm{E}_{N, p} N_{(G, H)}\left(\widetilde{x}_{1}, \ldots, \widetilde{x}_{k}\right) & \leqslant N_{(G, H)}\left(\widetilde{x}_{1}, \ldots, \widetilde{x}_{k}\right) \\
& \leqslant(1+\varepsilon) \mathrm{E}_{N, p} N_{(G, H)}\left(\widetilde{x}_{1}, \ldots, \widetilde{x}_{k}\right) .
\end{aligned}
$$

При этом

$$
\mathrm{E}_{N, p} N_{(G, H)}\left(\widetilde{x}_{1}, \ldots, \widetilde{x}_{k}\right)=\Theta\left(N^{f_{\alpha}(G, H)}\right) .
$$


В дальнейшем для двух функций $f=f(N)$ и $g=g(N)$, стремящихся к бесконечности при $N \rightarrow \infty$, мы будем использовать обозначение $f \sim g$, если для любого $\varepsilon>0$ при достаточно больших $N$ выполнено соотношение $(1-\varepsilon) f(N) \leqslant g(N) \leqslant(1+\varepsilon) f(N)$.

Помимо теоремы 12 Дж. Спенсер и С. Шела (см. [8], [50]) для исследования законов нуля или единицы доказали теорему о количестве максимальных расширений подграфов в случайном графе (для случая "запрещенных" жестких пар). Более формально, пусть случайная величина $N_{(G, H)}^{(K, T)}\left(\widetilde{x}_{1}, \ldots, \widetilde{x}_{k}\right)$ ставит в соответствие каждому графу $\mathscr{G}$ из $\Omega_{N}$ количество таких точных $(G, H)$-расширений $\widetilde{G}$ графа $\widetilde{H}=\left.\mathscr{G}\right|_{\left\{\widetilde{x}_{1}, \ldots, \widetilde{x}_{k}\right\}}$, что пара $(\widetilde{G}, \widetilde{H})$ является $(K, T)$-максимальной в $\mathscr{G}$. Сформулируем теорему, доказанную в работе [50], об асимптотическом поведении этой случайной величины.

Теорема 13 (Дж. Спенсер, С. Шела, 1988, [50]). Пусть пара $(K, T)$ является $\alpha$-жесткой. Тогда с асимптотической вероятностъю 1 для любых вериин $\widetilde{x}_{1}, \ldots, \widetilde{x}_{k}$ выполнено

$$
\begin{aligned}
N_{(G, H)}^{(K, T)}\left(\widetilde{x}_{1}, \ldots, \widetilde{x}_{k}\right) & \sim N_{(G, H)}\left(\widetilde{x}_{1}, \ldots, \widetilde{x}_{k}\right) \\
& \sim \mathrm{E}_{N, p} N_{(G, H)}^{(K, T)}\left(\widetilde{x}_{1}, \ldots, \widetilde{x}_{k}\right)=\Theta\left(N^{f_{\alpha}(G, H)}\right) .
\end{aligned}
$$

Сформулируем, наконец, утверждение об ограниченности количества $\alpha$-жестких расширений в случайном графе. Пусть $r, t \in \mathbb{N}$ - произвольные числа. Последовательность графов $H_{1}, G_{1}, \ldots, H_{i}, G_{i}$ называется $\alpha$-жесткой $(r, t)$-ие$n ъ ю$, если она удовлетворяет следующим условиям: $H_{1} \subset G_{1}$, пары $\left(G_{j}, H_{j}\right)$ являются $\alpha$-жесткими, $v\left(G_{j}, H_{j}\right) \leqslant t$ для всех $j \in\{1, \ldots, i\}, H_{j} \subset G_{1} \cup \cdots \cup G_{j-1}$, $G_{j} \cap\left(G_{1} \cup \cdots \cup G_{j-1}\right)=H_{j}$ для всех $j \in\{2, \ldots, i\}, v\left(H_{1}\right)=r$. Также рассматривается "вырожденный" случай $t=0$. В этом случае $i=1$ и $H_{1}=G_{1}$ (хотя в основном определении включение $H_{1} \subset G_{1}$ предполагалось строгим).

Лемма 2 (Дж. Спенсер, С. Шела, 1988, [50]). Существует число $K_{t}(r)$ maкое, что с вероятностъю, стремящейся $\kappa 1$, для любых вериин $\widetilde{x}_{1}, \ldots, \widetilde{x}_{r} \in V_{N}$ выполнено следующее свойство. Если $H_{1}, G_{1}, \ldots, H_{i}, G_{i}$ - такая $\alpha$-жесткая $(r, t)$-цепь, что в графе $\mathscr{G} \in \Omega_{N}$ существует точное $\left(G_{1} \cup \cdots \cup G_{i}, H_{1}\right)$-расширение графа $\left.\mathscr{G}\right|_{\left\{\widetilde{x}_{1}, \ldots, \widetilde{x}_{r}\right\}}, \operatorname{mov}\left(G_{1} \cup \cdots \cup G_{i}, H_{1}\right) \leqslant K_{t}(r)$.

ДокАЗАтЕЛьство. Пусть $m$ - такое натуральное число, что $[(1 / \alpha) v]+1>$ $(1 / \alpha) v+1 / m$ для любого $v \in\{1, \ldots, t\}$. Пусть, кроме того, $\widetilde{\Omega}_{N}-$ множество всех таких графов из $\Omega_{N}$, что в каждом из них не существует подграфа с плотностью, большей $1 / \alpha$, количество вершин которого не превосходит $2 m t^{2} r+r$. По теореме 3 имеем $\lim _{N \rightarrow \infty} \mathrm{P}_{N, p}\left(\widetilde{\Omega}_{N}\right)=1$.

Пусть $\mathscr{G} \in \widetilde{\Omega}_{N}, \widetilde{x}_{1}, \ldots, \widetilde{x}_{r} \in V_{N}$, а $i \in \mathbb{N}$ - некоторое количество пар $\left(G_{j}, H_{j}\right)$, $H_{j} \subset G_{j} \subset \mathscr{G}$, удовлетворяющих условию леммы. Тогда

$$
\rho\left(G_{1} \cup \cdots \cup G_{i}\right) \geqslant \frac{\left[(1 / \alpha) v_{1}\right]+1+\cdots+\left[(1 / \alpha) v_{i}\right]+1}{r+v_{1}+\cdots+v_{i}},
$$


где $v_{1}, \ldots, v_{i} \leqslant t$. Легко заметить, что при $i=2 m t r$ справедливы неравенства

$$
v\left(G_{1} \cup \cdots \cup G_{i}\right) \leqslant 2 m t^{2} r+r
$$

И

$$
\rho\left(G_{1} \cup \cdots \cup G_{i}\right)>\frac{1}{\alpha}-\frac{r}{r+i}+\frac{i / m}{r+t i}>\frac{1}{\alpha},
$$

что противоречит определению множества $\widetilde{\Omega}_{N}$. Следовательно, $i<2 m t r$. Поэтому в качестве $K_{t}(r)$ можно выбрать величину $2 m t^{2} r$. Лемма доказана.

Обратимся теперь к закону нуля или единицы для иррационального $\alpha$.

Теорема 14 (Дж. Спенсер, С. Шела, 1988, [50]). Пусть $\alpha>0$ - иррациональное число. Тогда случайный граф $G\left(N, N^{-\alpha}\right)$ подчиняется закону нуля или единицы.

ДокАЗАтЕЛЬСтво. Пусть $k \in \mathbb{N}$. Положим $a_{k}=0, a_{j}=j+1+K_{a_{j+1}}(j+1)$ для всех $j \in\{0, \ldots, k-1\}$, где $K_{t}(r)$ - величина, определенная в формулировке леммы 2. Для любого $N \in \mathbb{N}$ обозначим $\widetilde{\Omega}_{N}$ множество всех графов $X$ из $\Omega_{N}$, которые обладают следующими свойствами.

- В $X$ не существует подграфа с плотностью, большей $1 / \alpha$, количество вершин которого не превосходит $a_{0}$. Более того, для любого такого графа $H$, что $v(H) \leqslant a_{0}, \rho^{\max }(H)<1 / \alpha$, в $X$ найдется копия $H$.

- Пусть $(G, H)$ - $\alpha$-надежная пара, причем $v(G) \leqslant a_{0}+a_{1}$. Тогда в графе $X$ для любого подграфа $\widetilde{H}$ на $v(H)$ вершинах найдется такое точное $(G, H)$-расширение $\widetilde{G}$ графа $\widetilde{H}$, что пара $(\widetilde{G}, \widetilde{H})$ является $(K, T)$-максимальной для любой $\alpha$-жесткой пары $(K, T)$ с $v(K) \leqslant a_{1}+1$.

- Для любого $j \in\{1, \ldots, k\}$ и любой $\alpha$-жесткой $\left(j, a_{j}\right)$-цепи $H_{1}, G_{1}, \ldots, H_{i}, G_{i}$ в $X$ выполнено неравенство $v\left(G_{1} \cup \cdots \cup G_{i}\right) \leqslant a_{j-1}$.

По теореме 3 , теореме 13 и лемме 2 выполнено $\lim _{N \rightarrow \infty} \mathrm{P}_{N, p}\left(\widetilde{\Omega}_{N}\right)=1$. Пусть $N, M \in \mathbb{N}, A \in \widetilde{\Omega}_{N}, B \in \widetilde{\Omega}_{M}$. В силу теоремы 7 для доказательства теоремы 14 нам достаточно предъявить выигрышную стратегию Консерватора в игpe $\operatorname{EHR}(A, B, k)$. Вершину, выбранную в $j$-м раунде, $j \in\{1, \ldots, k\}$, мы будем обозначать $x_{j}$, если эта вершина выбрана в графе $A$, и $y_{j}$, если эта вершина выбрана в графе $B$.

Пусть в первом раунде Новатор выбрал некоторую вершину, скажем, в графе $A$. Пусть, кроме того, $H_{1}, G_{1}, \ldots, H_{i}, G_{i}$ - такая $\alpha$-жесткая $\left(1, a_{1}\right)$-цепь в $A$, где $H_{1}=\left(\left\{x_{1}\right\}, \varnothing\right)$, что граф $G_{1} \cup \cdots \cup G_{i}$ является $(K, T)$-максимальным для любой $\alpha$-жесткой пары $(K, T)$, удовлетворяющей условию $v(K, T) \leqslant a_{1}$. Выполнено неравенство

$$
v\left(G_{1} \cup \cdots \cup G_{i}\right) \leqslant 1+K_{a_{1}}(1)=a_{0} .
$$

Так как $A \in \widetilde{\Omega}_{N}$, то максимальная плотность графа $X_{1}:=G_{1} \cup \cdots \cup G_{i}$ меньше, чем $1 / \alpha$. Поэтому в силу определения множества $\widetilde{\Omega}_{M}$ в графе $B$ существует подграф $Y_{1}$, изоморфный графу $X_{1}$, являющийся $(K, T)$-максимальным в $B$ для любой такой пары $(K, T)$, что $v(K, T) \leqslant a_{1}$. Консерватор выберет вершину $y_{1}$, являющуюся образом вершины $x_{1}$ при изоморфизме $X_{1} \rightarrow Y_{1}$. 
Пусть $j \in\{1, \ldots, k-1\}$ - количество сыгранных раундов. Пусть, кроме того, в графах $A$ и $B$ выбраны подграфы $X_{j}$ и $Y_{j}$ соответственно, которые обладают следующими свойствами.

(j.1) Вершины $x_{1}, \ldots, x_{j}$ принадлежат графу $X_{j}$, вершины $y_{1}, \ldots, y_{j}$ принадлежат графу $Y_{j}$.

(j.2) Графы $X_{j}$ и $Y_{j}$ изоморфны, $v\left(X_{j}\right)=v\left(Y_{j}\right) \leqslant a_{j-1}$.

$(j .3)$ Графы $X_{j}$ и $Y_{j}$ являются $(K, T)$-максимальным в $A$ и $B$ соответственно для любой пары $(K, T)$, удовлетворяющей условию $v(K, T) \leqslant a_{j}$.

Очевидно, графы $X_{1}$ и $Y_{1}$ обладают свойствами (1.1), (1.2) и (1.3). Докажем, что, какую бы вершину $\left(x_{j+1}\right.$ или $\left.y_{j+1}\right)$ ни выбрал Новатор в $(j+1)$-м раунде, Консерватор сможет найти такую вершину $\left(y_{j+1}\right.$ или $\left.x_{j+1}\right)$ и графы $X_{j+1} \subset A$, $Y_{j+1} \subset B$, что они обладают свойствами $(j+1.1),(j+1.2)$ и $(j+1.3)$.

Если в $(j+1)$-м раунде Новатор выбрал вершину, лежащую внутри графа $X_{j}$ (графа $Y_{j}$ ), Консерватор сможет найти вершину, являющуюся ее образом при изоморфизме $X_{j} \rightarrow Y_{j}\left(Y_{j} \rightarrow X_{j}\right)$. В этом случае положим $X_{j+1}=X_{j}$, $Y_{j+1}=Y_{j}$.

Пусть в $(j+1)$-м раунде Новатор выбрал некоторую вершину $x_{j+1}$ вне графа $X_{j}$ (без ограничения общности будем считать, что Новатор выбрал вершину в графе $A$ ). Пусть, кроме того, $H_{1}, G_{1}, \ldots, H_{i}, G_{i}$ - такая $\alpha$-жесткая $\left(j+1, a_{j+1}\right)$-цепь, что $H_{1}=\left.A\right|_{\left\{x_{1}, \ldots, x_{j+1}\right\}}$ и граф $X_{j+1}:=G_{1} \cup \cdots \cup G_{i}$ является $(K, T)$-максимальным для любой пары $(K, T)$, удовлетворяющей условию $v(K, T) \leqslant a_{j+1}$. Так как $A \in \widetilde{\Omega}_{N}$, то $v\left(X_{j+1}\right) \leqslant a_{j}$.

Предположим, что в графе $X_{j+1}$ существует такой подграф $S$, содержащий $\left.A\right|_{\left\{x_{1}, \ldots, x_{j}\right\}}$, что $f_{\alpha}\left(S \cup X_{j}, X_{j}\right)<0$. Тогда без ограничения общности можно считать, что в $S$ нет подграфов, обладающих тем же свойством. Иными словами, для любого подграфа $\widetilde{S}$ в $S$, содержащего $\left.A\right|_{\left\{x_{1}, \ldots, x_{j}\right\}}$, выполнено неравенство $f_{\alpha}\left(\widetilde{S} \cup X_{j}, X_{j}\right)>0$ (неравенство строгое, так как $\alpha$ - иррациональное число). Поэтому $f_{\alpha}\left(S \cup X_{j}, \widetilde{S} \cup X_{j}\right)<0$. Таким образом, пара $\left(S \cup X_{j}, X_{j}\right)$ является $\alpha$-жесткой. Получили противоречие со свойством $(j .3)$ графа $X_{j}$. Следовательно, $f_{\alpha}\left(S \cup X_{j}, X_{j}\right)>0$ для любого такого графа $S$, что $\left.A\right|_{\left\{x_{1}, \ldots, x_{j}\right\}} \subset S \subseteq X_{j+1}$, $S \cap X_{j+1} \neq S$, т. е. пара $\left(X_{j+1} \cup X_{j}, X_{j}\right)$ является $\alpha$-надежной.

Так как $B \in \widetilde{\Omega}_{M}$ и $v\left(X_{j} \cup X_{j+1}\right) \leqslant a_{j-1}+a_{j} \leqslant a_{0}+a_{1}$, то в графе $B$ найдется такое точное $\left(X_{j+1} \cup X_{j}, X_{j}\right)$-расширение $Y$ графа $Y_{j}$, что пара $\left(Y, Y_{j}\right)$ является $(K, T)$-максимальной для любой пары $(K, T)$, удовлетворяющей условию $v(K, T) \leqslant a_{j+1}$. Тогда существуют такие граф $Y_{j+1} \subset Y$ и изоморфизм $f: X_{j+1} \cup X_{j} \rightarrow Y$, переводящий граф $X_{j+1}$ в $Y_{j+1}$, что $f\left(x_{1}\right)=y_{1}, \ldots, f\left(x_{j}\right)=y_{j}$. Положим $y_{j+1}=f\left(x_{j+1}\right)$. Так как граф $Y_{j}$ обладает свойством $(j .3)$ и $a_{j}<a_{j+1}$, то граф $Y_{j}$ является $(K, T)$-максимальным для любой пары $(K, T)$, удовлетворяющей условию $v(K, T) \leqslant a_{j+1}$. Следовательно, граф $Y$ также является $(K, T)$-максимальным для любой пары $(K, T)$, удовлетворяющей условию $v(K, T) \leqslant a_{j+1}$.

Чтобы доказать, что графы $X_{j+1}$ и $Y_{j+1}$ обладают свойствами $(j+1.1)$, $(j+1.2)$ и $(j+1.3)$, осталось обосновать $(K, T)$-максимальность графа $Y_{j+1}$ для любой пары $(K, T)$, удовлетворяющей условию $v(K, T) \leqslant a_{j+1}$. Предположим 
противное. Тогда существует такой граф $Z$ на не более чем $a_{j+1}$ вершинах, имеющий непустое пересечение с $Y_{j}$, что $Y_{j+1} \cap Z=\varnothing$ и пара $\left(Z \cup Y_{j+1}, Y_{j+1}\right)$ является $\alpha$-жесткой. Если граф $Z$ не содержится целиком в $Y$, то пара $(Z \cup Y, Y)$ является $\alpha$-жесткой и мы приходим к противоречию с максимальностью графа $Y$. Если же $Z$ целиком содержится в $Y$, то обозначим $\widetilde{Z}$ граф, являющийся образом графа $Z$ при отображении $f^{-1}$. Так как пара $\left(\widetilde{Z} \cup X_{j+1}, X_{j+1}\right)$ является $\alpha$-жесткой, то приходим к противоречию с максимальностью графа $X_{j+1}$. Таким образом, граф $Y_{j+1}$ действительно является $(K, T)$-максимальным для любой пары $(K, T)$, удовлетворяющей условию $v(K, T) \leqslant a_{j+1}$.

$\mathrm{K}$ концу игры выбраны вершины $x_{1}, \ldots, x_{k} \in V(A), y_{1}, \ldots, y_{k} \in V(B)$, а также такие графы $X_{k} \subset A$ и $Y_{k} \subset B$, что вершины $x_{1}, \ldots, x_{k}$ принадлежат графу $X_{k}$, вершины $y_{1}, \ldots, y_{k}$ принадлежат графу $Y_{k}$ и графы $X_{k}$ и $Y_{k}$ изоморфны. Следовательно, Консерватор выигрывает игру. Теорема доказана.

\section{6. Некоторые обобщения результатов из разделов 4 и 5}

В данном разделе мы приводим (без доказательств) известные результаты, относящиеся к вероятностям свойств первого порядка случайных графов в различных моделях. Эти результаты обобщают и дополняют теоремы, сформулированные в предыдущих двух разделах.

В разделе 5 мы доказали, что случайный граф $G\left(N, N^{-\alpha}\right)$ не подчиняется закону нуля или единицы, если $\alpha$ - рациональное число из интервала $(0,1)$. Возникает естественный вопрос: можно ли немного "сдвинуть" функцию $p=N^{-\alpha}$ таким образом, чтобы закон был выполнен? В 1991 г. Дж. Спенсер и Т. Лучак доказали, что для любого рационального $\alpha \in(0,1)$ существует такая функция $p=N^{-\alpha+o(1)}$, что случайный граф $G(N, p)$ подчиняется закону нуля или единицы.

Теорема 15 (Дж. Спенсер, Т. Лучак, 1991, [55]). Пусть $a, b, a<b,-$ взаимно простые натуральные числа. Пусть, кроме того,

$$
p_{1}(N)=\left((b-a+1)(\log N+\omega(N) \log \log N) N^{-a}\right)^{1 / b},
$$

где $\omega$ - такая стремящаяся к бесконечности функция, что $p_{1}(N)=N^{-a / b+o(1)}$. Тогда случайный граф $G\left(N, p_{1}\right)$ подчиняется закону нуля или единиць. Более того, существует такая функиия $p_{2}=p_{2}(N)$, удовлетворяющая условиям $p_{2}(N)<N^{-a / b}$ и $p_{2}(N)=N^{-a / b+o(1)}$, что случайный граф $G\left(N, p_{2}\right)$ подчиняется закону нуля или единицы.

Рассмотрим произвольное число $\alpha \in \mathbb{Q} \cap(0,1)$ и случайный граф $G\left(N, N^{-\alpha}\right)$. Для всех ли свойств первого порядка существует предел вероятности этих свойств? Ответ на этот вопрос был получен в 1988 г. Дж. Спенсером и С. Шела.

Теорема 16 (Дж. Спенсер, С. Шела, 1988, [50]). Для любого $\alpha \in \mathbb{Q} \cap(0,1)$ существует такое свойство первого порядка L, что последовательность $\left\{\mathrm{P}_{N, N^{-\alpha}}(L)\right\}_{N \in \mathbb{N}}$ не сходится. 
Более того, в этой работе доказано, что для любого $\alpha \in \mathbb{Q} \cap(0,1)$ существуют такие стремящиеся к бесконечности функции $\beta_{1}(N)$ и $\beta_{2}(N)$ и такое свойство первого порядка $L$, что сходимость вероятности этого свойства отсутствует и при

$$
N^{-\alpha} / \beta_{1}<p<N^{-\alpha} \beta_{2} .
$$

В 1992 г. Дж. Линч частично ответил на поставленный выше вопрос в случае $\alpha \geqslant 1$.

Теорема 17 (Дж. Линч, 1992, [56]). Пусть $l \in \mathbb{N}, \alpha=(l+1) / l$. Тогда для любого свойства первого порядка $L$ существует $\lim _{N \rightarrow \infty} \mathrm{P}_{N, N^{-\alpha}}(L)$. Если же $p=c / N$, где $c$ - произвольное положительное число, то для любого свойства первого порядка $L$ предел $\lim _{N \rightarrow \infty} \mathrm{P}_{N, N^{-\alpha}}(L)$ существует и может быть въражен функиией от с, использующей в своей записи само число с, сложение, умножение, деление и степени числа е.

Вернемся теперь к закону нуля или единицы и зададимся следующим вопросом. Будет ли случайный граф $G\left(N, N^{-\alpha}\right)$ подчиняться закону нуля или единицы при ограничении кванторной глубины формул первого порядка для каких-нибудь рациональных $\alpha$ из интервала $(0,1)$ ? Этот вопрос впервые был задан в работе М. Макартур, где был получен следующий результат.

Пусть $k \in \mathbb{N}$. Пусть, кроме того, $\mathscr{L}_{k}^{\infty}-$ множество свойств первого порядка, записываемых с помощью формул, содержащих бесконечное количество логических связок, кванторная глубина которых ограничена числом $k$.

Tеорема 18 (М. Макартур, 1997, [57]). Если $\alpha \in(0,1 /(k-1))$, то для любого свойства $L \in \mathscr{L}_{k}^{\infty}$ предел $\lim _{N \rightarrow \infty} \mathrm{P}_{N, N^{-\alpha}}(L)$ равен либо 0 , либо 1. При $\alpha=1 /(k-1)$ этот закон нарушается.

Исследованию законов нуля или единицы для свойств, выражаемых конечными предложениями с ограниченной кванторной глубиной, посвящен следующий раздел нашей работы.

Другие работы, в которых изучалось предельное поведение вероятностей свойств первого порядка случайных графов, были посвящены в основном доказательству закона нуля или единицы в моделях, отличных от модели Эрдёша-Реньи. В 1987 г. Ф. Колайтис, Х. Прёмел, Б. Ротшилд доказали закон нуля или единицы для графов, свободных от полных графов $K_{l+1}$. Сформулируем этот результат. Пусть $\mathscr{G}_{N}-$ множество всех графов, не содержащих $K_{l+1}$, на множестве вершин $\{1, \ldots, N\}$. На $\left(\mathscr{G}_{N}, 2^{\mathscr{G}_{N}}\right)$ зададим равномерное распределение вероятностей $\mathrm{P}_{N}$ :

$$
\mathrm{P}_{N}(X)=\frac{|X|}{\left|\mathscr{G}_{N}\right|}, \quad X \subseteq \mathscr{G}_{N}
$$

Теорема 19 (Ф. Колайтис, Х. Прёмел, Б. Ротшилд, 1987, [58]). Для любого свойства первого порядка $L$ выполнено

$$
\lim _{N \rightarrow \infty} \mathrm{P}_{N}(L) \in\{0,1\} .
$$


В 2007 г. Р. Гилман, Ю. Гуревич, А. Мясников доказали закон нуля или единицы для бесконечного графа. Пусть $X$ - связный граф, множество вершин которого бесконечно. Для любого натурального числа $n$ обозначим $B_{n}(X)$ индуцированный подграф в $X$ на множестве вершин, до которых существует путь от вершины $x$ длины, не превосходящей $n$. Будем говорить, что грая $X$ noдчиняется закону нуля или единицы, если этому закону для любого $x \in V(X)$ подчиняется случайный граф, имеющий равномерное распределение на множестве индуцированных подграфов в $B_{n}(X)$. Как доказали Гилман, Гуревич и Мясников, закон нуля или единицы верен, если граф $X$ обладает двумя свойствами: ограниченной степенью и свойством дублированных подграфов. Определим эти свойства. Будем говорить, что граф $X$ имеет ограниченную степень, если существует такое число $K$, что для любой вершины $x$ графа $X$ ее степень не превосходит $K$. Граф $X$ обладает свойством дублированных подграфов, если для любого его конечного подграфа $A$ существует такой подграф $B \subset X$, изоморфный $A$, что множества вершин графов $A$ и $B$ не пересекаются и между вершинами графа $A$ и вершинами графа $B$ нет ребер графа $X$.

Теорема 20 (Р. Гилман, Ю. Гуревич, А. Мясников, 2007, [59]). Пустъ X бесконечный связный граф с ограниченной степенъю, обладающий свойством дублированных подграфов. Тогда $X$ подчиняется закону нуля или единичь.

\section{7. Ограничение кванторной глубины}

Как мы доказали в разделе 5 , случайный граф $G\left(N, N^{-\alpha}\right)$ не подчиняется закону нуля или единицы при рациональных $\alpha$, принадлежащих интервалу $(0,1]$. Оказывается, закон нуля или единицы становится выполнен и при некоторых рациональных $\alpha$ из интервала $(0,1]$ при ограничении кванторной глубины формул первого порядка. Для любой последовательности графов $G_{n}$ будем говорить, что случайный граф $\mathscr{G}\left(G_{n}, p\right)$ подчиняется $k$-закону нуля или единицы, если для любого свойства, выражаемого формулой первого порядка, кванторная глубина которой не превосходит $k$, вероятность того, что случайный граф $\mathscr{G}\left(G_{n}, p\right)$ обладает этим свойством, стремится либо к 0 , либо к 1 . Для доказательства $k$-законов нуля или единицы используется следствие из теоремы 6 , доказательство которого аналогично доказательству теоремы 7 .

ТЕОрема 21. Случайный граф $G(N, p)$ подчиняется $k$-закону нуля или единицы тогда и только тогда, когда

$$
\begin{aligned}
\lim _{N, M \rightarrow \infty} \mathrm{P}_{N, M, p}(\{(A, B): & \text { у Консерватора есть выигрышная } \\
& \text { стратегия в игре } \operatorname{EHR}(A, B, k)\})=1 .
\end{aligned}
$$

ДокАЗАТЕльство дословно повторяет доказательство теоремы 7 с однимединственным изменением: нужно рассматривать не произвольное $k$, а именно то значение $k$, для которого доказывается теорема. 
В 2012 г. мы доказали, что случайный граф $G\left(N, N^{-\alpha}\right)$ подчиняется $k$-закону нуля или единицы при рациональных $\alpha$ из интервала $(0,1 /(k-2))$.

Теорема 22 (М.Е. Жуковский, 2012, [60]). Пусть $p=N^{-\alpha}, 0<\alpha<$ $1 /(k-2)$. Тогда случайный граф $G(N, p)$ подчиняется $k$-закону нуля или единицы.

ДокАЗАТЕЛЬСтво мы приводим только для случая рациональных $\alpha$, так как справедливость закона при иррациональных $\alpha$ утверждает теорема 14 . Введем некоторые вспомогательные определения, которые мы будем использовать в доказательстве и далее в этом разделе.

Рассмотрим произвольные графы $T, K$. Пусть $T \subset K$, любая вершина графа $T$ соединена с некоторой вершиной из $V(K) \backslash V(T)$ и для любого такого графа $S$, что $T \subset S \subset K$, справедливо неравенство $v(S, T)-\alpha \cdot e(S, T)>0$, но $v(K, T)-\alpha \cdot e(K, T)=0$. В этом случае пару $(K, T)$ будем называть $\alpha$-нейтралъной. Рассмотрим произвольные вершины $\widetilde{x}_{1}, \ldots, \widetilde{x}_{k} \in V_{N}$. Для произвольной пары графов $(G, H)$, где $H \subset G$, рассмотрим случайную величину $\widehat{N}_{(G, H)}^{(K, T)}\left(\widetilde{x}_{1}, \ldots, \widetilde{x}_{k}\right)$, которая ставит в соответствие каждому графу $\mathscr{G} \in \Omega_{N}$ количество таких точных $(G, H)$-расширений $\widetilde{G}$ графа $\widetilde{H}=\left.\mathscr{G}\right|_{\left\{\widetilde{x}_{1}, \ldots, \widetilde{x}_{k}\right\}}$, что пара $(\widetilde{G}, \widetilde{H})$ является $(K, T)$-максимальной в $\mathscr{G}$. Для этих случайных величин выполнен результат, подобный теореме 13.

Теорема 23 (М.Е. Жуковский, 2012, [61]). Пусть пара $(G, H)$ является $\alpha$-надежной, пара $(K, T)$ - $\alpha$-нейтральной. Тогда с асимптотической вероятностью 1 для любых вершин $\widetilde{x}_{1}, \ldots, \widetilde{x}_{k}$ выполнено

$$
\widehat{N}_{(G, H)}^{(K, T)}\left(\widetilde{x}_{1}, \ldots, \widetilde{x}_{k}\right) \sim \mathrm{E}_{N, p} \widehat{N}_{(G, H)}^{(K, T)}\left(\widetilde{x}_{1}, \ldots, \widetilde{x}_{k}\right)=\Theta\left(N^{f_{\alpha}(G, H)}\right) .
$$

\section{Рассмотрим множества}

$$
\left(0, \frac{1}{k-1}\right), \quad\left\{\frac{1}{k-1}\right\}, \quad\left(\frac{1}{k-1}, \frac{2}{2 k-3}\right), \quad\left\{\frac{2}{2 k-3}\right\}, \quad\left(\frac{2}{2 k-3}, \frac{1}{k-2}\right) .
$$

В зависимости от того, какому из этих множеств принадлежит число $\alpha$, доказательство теоремы 22 разобьется на случаи.

1) Пусть $\alpha<1 /(k-1)$. Пусть, кроме того, $\mathscr{E} \in \mathscr{F}_{N}-$ множество графов, обладающих свойством полного расширения уровня $k-1$ (см. раздел 4). Покажем, что $\lim _{N \rightarrow \infty} \mathrm{P}_{N, p}(\mathscr{E})=1$. Справедливы соотношения

$$
\mathrm{P}_{N, p}\left(\Omega_{N} \backslash \mathscr{E}\right) \leqslant(k-1)^{2} N^{k-1}\left(1-p^{k-1}\right)^{N} \leqslant(k-1)^{2} N^{k-1} \exp \left(-N^{1-(k-1) \alpha}\right) \rightarrow 0,
$$

т. е. действительно $\lim _{N \rightarrow \infty} \mathrm{P}_{N, p}(\mathscr{E})=1$. Но раз с вероятностью, стремящейся к 1 , выполнено свойство полного расширения уровня $k-1$, то, очевидно, с вероятностью $\mathrm{P}_{N, M, p}$, стремящейся к 1 , в игре $\operatorname{EHR}(G(N, p(N)), G(M, p(M)), k)$ у Консерватора есть выигрышная стратегия. Следовательно, в силу теоремы 21 выполнен $k$-закон нуля или единицы. 
2) Пусть $\alpha=1 /(k-1)$. Из предыдущего пункта, очевидно, следует, что с вероятностью, стремящейся к 1 , выполнено свойство полного расширения уровня $k-2$. Тогда для $k-2$ ходов у Консерватора с вероятностью $\mathrm{P}_{N, M, p}$, стремящейся к 1 , есть выигрышная стратегия в игре $\operatorname{EHR}(G(N, p(N)), G(M, p(M)), k)$.

Заметим, что если $H \subset G$ и $v(G, H)=1, v(H)=k-2$, то пара $(G, H)$ является $\alpha$-надежной. Действительно, $e(G, H) \leqslant k-2$ и $1-\alpha(k-2)>0$. Аналогично, если $v(G, H)=2, v(H)=k-2$, то пара $(G, H)$ также является $\alpha$-надежной. Если же $v(G, H)=1, v(H)=k-1$, то пара $(G, H)$ является либо $\alpha$-надежной, либо $\alpha$-нейтральной. При этом $\alpha$-нейтральной она является тогда и только тогда, когда $e(G, H)=k-1$. Рассмотрим такую $\alpha$-нейтральную пару $(K, T)$.

Пусть графы $A, B$ обладают следующим свойством. Для любой такой $\alpha$-надежной пары $(G, H)$, что $v(G) \leqslant k$, и любых подграфов $\widetilde{H}_{A} \subset A, \widetilde{H}_{B} \subset B$ на $v(H)$ вершинах в графах $A$ и $B$ существуют такие $(K, T)$-максимальные точные $(G, H)$-расширения $\widetilde{G}_{A}$ и $\widetilde{G}_{B}$ графов $\widetilde{H}_{A}$ и $\widetilde{H}_{B}$ соответственно, что каждая из пар $\left(\widetilde{G}_{A}, \widetilde{H}_{A}\right)$ и $\left(\widetilde{G}_{B}, \widetilde{H}_{B}\right)$ является $(K, T)$-максимальной.

Пусть в игре $\operatorname{EHR}(A, B, k)$ Новатором и Консерватором выбраны вершины $x_{1}, \ldots, x_{k-2}$ в графе $A$ и вершины $y_{1}, \ldots, y_{k-2}$ в графе $B$. Пусть, кроме того, Консерватор одерживает победу, т. е. подграфы, индуцированные на множества выбранных вершин, изоморфны. В силу теорем 21 и 23, если независимо от выбора еще двух вершин Новатором Консерватор сможет победить, то случайный граф $G(N, p)$ подчиняется $k$-закону нуля или единицы.

Итак, пусть Новатор выбрал вершину $x_{k-1}$, например, в графе $A$. У выбранного графа $\left.A\right|_{\left\{x_{1}, \ldots, x_{k-1}\right\}}$ либо существует $(K, T)$-расширение в $A$, либо нет. Если оно существует (предположим, его образует вершина $x_{k}$ ), то пара $\left(\left.A\right|_{\left\{x_{1}, \ldots, x_{k}\right\}},\left.A\right|_{\left\{x_{1}, \ldots, x_{k-2}\right\}}\right)$ является $\alpha$-надежной. Следовательно, Консерватор сможет найти такие вершины $y_{k-1}, y_{k} \in V(B)$, что граф $\left.B\right|_{\left\{y_{1}, \ldots, y_{k}\right\}}$ является точным $\left(\left.A\right|_{\left\{x_{1}, \ldots, x_{k}\right\}},\left.A\right|_{\left\{x_{1}, \ldots, x_{k-2}\right\}}\right)$-расширением графа $\left.B\right|_{\left\{y_{1}, \ldots, y_{k-2}\right\}}$. Тогда на $(k-1)$-м ходу Консерватор выбирает вершину $y_{k-1}$. Далее, если Новатор выберет вершину, соединенную с каждой из $k-1$ выбранных, то Консерватор сможет найти вершину, соединенную с каждой из выбранных в своем графе. Если же он выберет, скажем, вершину $y$, не соединенную с какой-нибудь из $y_{1}, \ldots, y_{k-1}$, то пара $\left(\left.B\right|_{\left\{y_{1}, \ldots, y_{k-1}, y\right\}},\left.B\right|_{\left\{y_{1}, \ldots, y_{k-1}\right\}}\right)$ является $\alpha$-надежной и Консерватор победит в силу определения графа $A$.

Если же $(K, T)$-расширения не существует, то Консерватор сможет выбрать такую подходящую ему вершину $y_{k-1}$ (т. е. $\forall i \in\{1,2, \ldots, k-2\}\left(\left(x_{i} \sim x_{k-1}\right) \Leftrightarrow\right.$ $\left.\left.\left(y_{i} \sim y_{k-1}\right)\right)\right)$, что у графа $\left.B\right|_{\left\{y_{1}, \ldots, y_{k-1}\right\}}$ не существует $(K, T)$-расширения в $B$. Дальнейшая выигрышная стратегия Консерватора очевидна.

3) Пусть $1 /(k-1)<\alpha<2 /(2 k-3)$. С вероятностью, стремящейся к 1 , выполнено свойство полного расширения уровня $k-2$. Тогда для $k-2$ ходов у Консерватора с вероятностью $\mathrm{P}_{N, M, p}$, стремящейся к 1 , есть выигрышная стратегия в игре $\operatorname{EHR}(G(N, p(N)), G(M, p(M)), k)$. 
Если для графа $H$ выполнено равенство $v(H)=k-2$, то для любого графа $G$ на $k$ вершинах, содержащего $H$, пара $(G, H)$ является $\alpha$-надежной и для любого графа $G$ на $k-1$ вершинах, содержащего $H$, пара $(G, H)$ также является $\alpha$-надежной. Если же $v(H)=k-1$, то существует такой граф $G$ на $k$ вершинах, содержащий $H$, что пара $(G, H)$ является $\alpha$-жесткой (пара $(G, H)$ будет $\alpha$-жесткой тогда и только тогда, когда $e(G, H)=k-1)$. Обозначим такую пару $(K, T)$. В остальных случаях (при том же значении $v(H))$ пара $(G, H)$ является $\alpha$-надежной.

Пусть графы $A, B$ обладают следующим свойством. Для любой такой $\alpha$-надежной пары $(G, H)$, что $v(G) \leqslant k$, и любых подграфов $\widetilde{H}_{A} \subset A, \widetilde{H}_{B} \subset B$ на $v(H)$ вершинах в графах $A$ и $B$ существуют такие точные $(G, H)$-расширения $\widetilde{G}_{A}$ и $\widetilde{G}_{B}$ графов $\widetilde{H}_{A}$ и $\widetilde{H}_{B}$ соответственно, что каждая из пар $\left(\widetilde{G}_{A}, \widetilde{H}_{A}\right)$ и $\left(\widetilde{G}_{B}, \widetilde{H}_{B}\right)$ является $(K, T)$-максимальной.

Пусть в игре $\operatorname{EHR}(A, B, k)$ Новатором и Консерватором выбраны вершины $x_{1}, \ldots, x_{k-2}$ в графе $A$ и вершины $y_{1}, \ldots, y_{k-2}$ в графе $B$. Пусть, кроме того, Консерватор одерживает победу, т. е. подграфы, индуцированные на множества выбранных вершин, изоморфны. В силу теорем 13 и 21, если независимо от выбора еще двух вершин Новатором Консерватор сможет победить, то случайный граф $G(N, p)$ подчиняется $k$-закону нуля или единицы.

Дальнейшие рассуждения аналогичны рассуждениям из случая 2).

4) Пусть $\alpha=2 /(2 k-3)$. Аналогично предыдущим случаям для $k-3$ ходов у Консерватора с вероятностью $\mathrm{P}_{N, M, p}$, стремящейся к 1 , есть выигрышная стратегия в игре $\operatorname{EHR}(G(N, p(N)), G(M, p(M)), k)$.

Если $v(H)=k-2$, то для любого графа $G$ на $k$ вершинах, содержащего $H$, пара $(G, H)$ является либо $\alpha$-надежной, либо $\alpha$-нейтральной (пара $(G, H)$ будет $\alpha$-нейтральной тогда и только тогда, когда $e(G, H)=2 k-3)$, для любого графа $G$ на $k-1$ вершинах, содержащего $H$, пара $(G, H)$ является $\alpha$-надежной. Если же $v(H)=k-1$, а $v(G)=k$, то пара $(G, H)$ является либо $\alpha$-надежной, либо $\alpha$-жесткой (пара $(G, H)$ будет $\alpha$-жесткой тогда и только тогда, когда $e(G, H)=k-1)$.

Определим графы $T_{1}, T_{2}, K_{1}, K_{2}$. Пусть $T_{1} \subset K_{1}, T_{2} \subset K_{2}$. Пусть, кроме того, $v\left(T_{1}\right)=k-2, v\left(K_{1}, T_{1}\right)=2, v\left(T_{2}\right)=k-1, v\left(K_{2}, T_{2}\right)=1$ и пара $\left(K_{1}, T_{1}\right)$ является $\alpha$-нейтральной, а пара $\left(K_{2}, T_{2}\right)-\alpha$-жесткой.

Пусть графы $A, B$ обладают следующим свойством. Для любой такой $\alpha$-надежной пары $(G, H)$, что $v(G) \leqslant k$, и любых подграфов $\widetilde{H}_{A} \subset A, \widetilde{H}_{B} \subset B$ на $v(H)$ вершинах в графах $A$ и $B$ существуют такие точные $(G, H)$-расширения $\widetilde{G}_{A}$ и $\widetilde{G}_{B}$ графов $\widetilde{H}_{A}$ и $\widetilde{H}_{B}$ соответственно, что каждая из пар $\left(\widetilde{G}_{A}, \widetilde{H}_{A}\right)$ и $\left(\widetilde{G}_{B}, \widetilde{H}_{B}\right)$ является $\left(K_{1}, T_{1}\right)$-максимальной и $\left(K_{2}, T_{2}\right)$-максимальной.

Пусть в игре $\operatorname{EHR}(A, B, k)$ Новатором и Консерватором выбраны вершины $x_{1}, \ldots, x_{k-3}$ в графе $A$ и вершины $y_{1}, \ldots, y_{k-3}$ в графе $B$. Пусть, кроме того, Консерватор одерживает победу, т. е. подграфы, индуцированные на множества выбранных вершин, изоморфны. В силу теорем 13, 21 и 23, если независимо от выбора еще трех вершин Новатором Консерватор сможет победить, то случайный граф $G(N, p)$ подчиняется $k$-закону нуля или единицы. 
Предположим, что Новатор выбрал вершину $x_{k-2}$, например, в графе $A$. У графа $\left.A\right|_{\left\{x_{1}, \ldots, x_{k-2}\right\}}$ либо существует $\left(K_{1}, T_{1}\right)$-расширение в $A$, либо не существует. Если его не существует, то Консерватор сможет выбрать такую подходящую ему вершину $y_{k-2}$, что у графа $\left.B\right|_{\left\{y_{1}, \ldots, y_{k-2}\right\}}$ нет $\left(K_{1}, T_{1}\right)$-расширений в $B$. Далее Новатор выбирает $(k-1)$-ю вершину. Если в соответствующем графе у подграфа, индуцированного на выбранные $k-1$ вершин, существует $\left(K_{2}, T_{2}\right)$-расширение, то выбранная $(k-1)$-я вершина и расширяющая $k$-я вершина образуют вместе с $k-2$ выбранными вершинами $\alpha$-надежную пару. Следовательно, Консерватор сможет найти нужную ему $(k-1)$-ю вершину (для которой тоже существует $\left(K_{2}, T_{2}\right)$-расширение) и победит. Если $\left(K_{2}, T_{2}\right)$-расширения не существует, то Консерватор сможет выбрать нужную вершину, так как в соответствующем графе найдется необходимая $\left(K_{2}, T_{2}\right)$-максимальная $\alpha$-надежная пара. Если для графа $\left.A\right|_{\left\{x_{1}, \ldots, x_{k-2}\right\}}$ существует $\left(K_{1}, T_{1}\right)$-расширение $\left.A\right|_{\left\{x_{1}, \ldots, x_{k}\right\}}$, то пара $\left(\left.A\right|_{\left\{x_{1}, \ldots, x_{k}\right\}},\left.A\right|_{\left\{x_{1}, \ldots, x_{k-3}\right\}}\right)$ является $\alpha$-надежной. Следовательно, Консерватор сможет найти такие вершины $y_{k-2}, y_{k-1}, y$, что вершина $y_{k-2}$ ведет его $\kappa$ победе (т. е. $\forall i \in\{1,2, \ldots, k-3\}\left(\left(x_{i} \sim x_{k-2}\right) \Leftrightarrow\left(y_{i} \sim\right.\right.$ $\left.\left.y_{k-2}\right)\right)$ ), а пара $\left(\left.B\right|_{\left\{y_{1}, \ldots, y_{k}\right\}},\left.B\right|_{\left\{y_{1}, \ldots, y_{k-2}\right\}}\right)$ является $\alpha$-нейтральной. Дальнейшие рассуждения аналогичны рассуждениям в случае, когда $\left(K_{1}, T_{1}\right)$-расширения не существует.

5) Пусть $2 /(2 k-3)<\alpha<1 /(k-2)$. Для $k-3$ ходов у Консерватора с вероятностью $\mathrm{P}_{N, M, p}$, стремящейся к 1 , есть выигрышная стратегия в игре $\operatorname{EHR}(G(N, p(N)), G(M, p(M)), k)$.

Если $v(H)=k-2$, то для любого графа $G$ на $k$ вершинах, содержащего $H$, пара $(G, H)$ является либо $\alpha$-надежной, либо $\alpha$-жесткой (пара $(G, H)$ будет $\alpha$-жесткой тогда и только тогда, когда $e(G, H)=2 k-3)$, для любого графа $G$ на $k-1$ вершинах, содержащего $H$, пара $(G, H)$ является $\alpha$-надежной. Если же $v(H)=k-1$, а $v(G)=k$, то пара $(G, H)$ является либо $\alpha$-надежной, либо $\alpha$-жесткой (пара $(G, H)$ будет $\alpha$-жесткой тогда и только тогда, когда $e(G, H)=$ $k-1)$. Рассмотрим $T_{1}, T_{2}, K_{1}, K_{2}$ и $A, B$ - графы из пункта 4$)$.

Пусть в игре $\operatorname{EHR}(A, B, k)$ Новатором и Консерватором выбраны вершины $x_{1}, \ldots, x_{k-3}$ в графе $A$ и вершины $y_{1}, \ldots, y_{k-3}$ в графе $B$. Пусть, кроме того, Консерватор одерживает победу, т. е. подграфы, индуцированные на множества выбранных вершин, изоморфны. В силу теоремы 13 и теоремы 21, если независимо от выбора еще трех вершин Новатором Консерватор сможет победить, то случайный граф $G(N, p)$ подчиняется $k$-закону нуля или единицы. Дальнейшие рассуждения аналогичны рассуждениям в случае 4). Теорема 22 доказана.

Мы также нашли ближайший к 1 интервал значений $\alpha$, при которых случайный граф $G\left(N, N^{-\alpha}\right)$ подчиняется $k$-закону нуля или единицы.

Теорема 24 (М. Е. Жуковский, 2013, [62]). Пустъ $k>3$ - произволъное натуральное число. Пусть, кроме того, $\mathscr{Q}$ - множество положительных дробей с числителем, не превосходящим числа $2^{k-1}$. Случайный граф $G\left(N, N^{-\alpha}\right)$ подчиняется $k$-закону нуля или единиць, если $\alpha=1-1 /\left(2^{k-1}+\beta\right), \beta \in(0, \infty) \backslash \mathscr{Q}$. 
ЗАмЕчАНИЕ 1. В этой теореме мы рассмотрели интервал $\left(1-2^{1-k}, 1\right)$ и получили множество рациональных чисел $\alpha$, при которых $k$-закон справедлив. Так как любое число из $\left(1-2^{1-k}, 1\right)$ представляется в виде $1-1 /\left(2^{k-1}+\beta\right)$, этот закон будет выполнен при любых $\alpha$ из

$$
\begin{aligned}
\left(1-\frac{1}{2^{k}}, 1\right) & \cup\left(1-\frac{1}{2^{k}-1}, 1-\frac{1}{2^{k}}\right) \cup \cdots \\
& \cup\left(1-\frac{1}{2^{k-1}+2^{k-2}}, 1-\frac{1}{2^{k-1}+2^{k-2}+1}\right) \\
& \cup\left(1-\frac{1}{2^{k-1}+\left(2^{k-1}-1\right) / 2}, 1-\frac{1}{2^{k-1}+2^{k-2}}\right) \cup \cdots \\
& \cup\left(1-\frac{1}{2^{k-1}+2^{k-1} / 3}, 1-\frac{1}{2^{k-1}+\left(2^{k-1}-\left[2^{k-1} / 3\right]\right) / 2}\right) \cup \cdots
\end{aligned}
$$

Длины интервалов уменьшаются при стремлении концов к $1-2^{1-k}$.

ДоКАЗАТЕЛЬСтво теоремы 24. Пусть $m \in \mathbb{N}$ - произвольное натуральное число. Рассмотрим такую пару графов $(G, H)$, что $G \supset H$. Будем говорить, что граф $G$ является $m$-расширением графа $H$ первого типа, если $m \geqslant 3$ и выполнено следующее условие. Существует такая вершина $x_{1}$ графа $G$, что

$$
\begin{aligned}
V(G) \backslash V(H)=\{ & \left.y_{1}^{1}, \ldots, y_{t_{1}}^{1}, y_{1}^{2}, \ldots, y_{t_{2}}^{2}\right\} \\
E(G) \backslash E(H)=\{ & \left\{x_{1}, y_{1}^{1}\right\},\left\{y_{1}^{1}, y_{2}^{1}\right\}, \ldots,\left\{y_{t_{1}-1}^{1}, y_{t_{1}}^{1}\right\},\left\{y_{t_{1}}^{1}, y_{1}^{2}\right\}, \\
& \left.\left\{y_{1}^{2}, y_{2}^{2}\right\}, \ldots,\left\{y_{t_{2}-1}^{2}, y_{t_{2}}^{2}\right\},\left\{y_{t_{2}}^{2}, y_{t_{1}}^{1}\right\}\right\},
\end{aligned}
$$

где $t_{1}+t_{2} \leqslant m-1, t_{1} \geqslant 0, t_{2} \geqslant 2$ и $\rho^{\max }(G)<m /(m-1)$ (при $t_{1}=0$ вершина $x_{1}$ является смежной с вершинами $\left.y_{1}^{2}, y_{t_{2}}^{2}\right)$. Граф $G$ мы называем $m$-расширением графа $H$ второго типа, если $m \geqslant 2$ и выполнено следующее условие. Существуют две такие различные вершины $x_{1}, x_{2}$ графа $G$, что

$$
G=\left(V(H) \sqcup\left\{y_{1}, \ldots, y_{t}\right\}, E(H) \sqcup\left\{\left\{x_{1}, y_{1}\right\},\left\{y_{1}, y_{2}\right\}, \ldots,\left\{y_{t-1}, y_{t}\right\},\left\{y_{t}, x_{2}\right\}\right\}\right),
$$

где $t \leqslant m-1$ и $\rho^{\max }(G)<m /(m-1)$. Граф $G$ является $m$-расширением графа $H$ mретъего типа, если $m \geqslant 2, V(H)=V(G), E(H) \subset E(G)$ и $\rho^{\max }(G)<$ $m /(m-1)$.

Для произвольного натурального числа $m \geqslant 3$ определим множество графов $\mathscr{H}_{m}$. Пусть $x$ - вершина. Граф без ребер на множестве вершин $\{x\}$ принадлежит $\mathscr{H}_{m}$. Далее, пусть $G \in \mathscr{H}_{m}$. Множество $\mathscr{H}_{m}$ содержит все попарно неизоморфные $m$-расширения первого, второго и третьего типов графа $G$.

Заметим, что любой граф $G$ из $\mathscr{H}_{m}$, отличный от $(\{x\}, \varnothing)$, содержит в себе конечный набор таких вложенных графов $G_{1}, \ldots, G_{t}, G_{0}=(\{x\}, \varnothing) \subset G_{1} \subset$ $\cdots \subset G_{t} \subseteq G$, что выполнены следующие свойства:

- $G_{i} \neq G_{i+1}$ для всех $i \in\{0,1, \ldots, t-1\}$;

- граф $G$ либо совпадает с $G_{t}$, либо является $m$-расширением третьего типа графа $G_{t}$, а графы $G_{i}$ являются $m$-расширениями первого или второго типа графов $G_{i-1}$ при $i \in\{0,1, \ldots, t\}$. 
Такую последовательность графов $G_{0}, G_{1}, \ldots, G_{t}, G$ будем называть $m$-разложением графа $G$.

Сформулируем утверждение о свойствах множества $\mathscr{H}_{m}$.

Лемма 3. Выполнены следующие свойства.

1. Пусть $G$ - граф из $\mathscr{H}_{m} u G_{0}, G_{1}, \ldots, G_{t}, G$-его т-разложение, причем либо $t=1$ и $G_{t} \neq G$, либо $t \geqslant 2$. Тогда найдутся такие натуральные числа $a, b$, umo $b \leqslant m u \rho^{\max }(G)=1+1 /(m-1+b / a)$.

2. Пусть $m \geqslant 2$ и $\rho \in(1, m /(m-1))$ - произвольное число. Тогда существует такое число $\eta \in \mathbb{N}$, что для любого натурального $v>\eta$ у любого графа $G \in \mathscr{H}_{m}$ на $v$ вершинах найдется подграф на не более чем $\eta$ вершинах с плотностъю, превосходящей $\rho$.

ДоКАЗАТЕЛЬСтво ЛЕммы начнем со свойства 1 . Если $t=1$, то $\rho\left(G_{1}\right)=1$, $v\left(G_{1}\right) \leqslant m$. Следовательно, $\rho(G)=\left(v\left(G_{1}\right)+e\right) / v\left(G_{1}\right)$, где $e \in \mathbb{N}$. Поэтому

$$
\rho(G)=1+\frac{1}{1+\left(v\left(G_{1}\right)-e\right) / e}<\frac{m}{m-1} .
$$

Если $e=1$, то $v\left(G_{1}\right)=m$ и $\rho(G)=1+1 /(m-1+1)$. Если $e>1$, то неравенство $v\left(G_{1}\right)>(m-1) e$ противоречит условию $v\left(G_{1}\right) \leqslant m$. Поэтому свойство 1 для рассмотренного случая доказано.

Пусть $t \geqslant 2$. Положим $v^{i+1}=v\left(G_{i+1}\right)-v\left(G_{i}\right), i \in\{0,1, \ldots, t-1\}$. Докажем, что

$$
\rho\left(G_{t}\right)=1+\frac{1}{m-1+b_{1} /(t-1)} \quad \text { при некотором } b_{1} \leqslant m .
$$

Справедливы соотношения

$$
\rho\left(G_{t}\right)=\frac{v^{1}+\cdots+v^{t}+t}{1+v^{1}+\cdots+v^{t}}=1+\frac{t-1}{1+v^{1}+\cdots+v^{t}}<1+\frac{1}{m-1} .
$$

Так как $v^{i} \leqslant m-1, i \in\{1, \ldots, t\}$, то

$$
m-1<\frac{1+v^{1}+\cdots+v^{t}}{t-1} \leqslant \frac{1+t(m-1)}{t-1}=m-1+\frac{m}{t-1} .
$$

Таким образом,

$$
1+\frac{1}{m-1+m /(t-1)} \leqslant \rho(G)<1+\frac{1}{m-1},
$$

при этом

$$
\rho(G)=1+\frac{1}{m-1+b_{1} /(t-1)},
$$

где $b_{1}=1+v^{1}+\cdots+v^{t}-(m-1)(t-1)$. Поэтому число $b_{1}$ не превосходит $m$.

Докажем теперь, что

$$
\rho(G)=1+\frac{1}{m-1+b_{2} /\left(t+e_{0}-1\right)}, \quad \text { где } \quad b_{2} \leqslant m, \quad e_{0}=e(G)-e\left(G_{t}\right) .
$$

Имеем

$$
\rho(G)=\frac{m(t-1)+b_{1}+e_{0}}{(m-1)(t-1)+b_{1}}=1+\frac{1}{m-1+\left(b_{1}-(m-1) e_{0}\right) /\left(t-1+e_{0}\right)} .
$$

Так как $\rho(G)<1+1 /(m-1)$, то $0<b_{2} \leqslant b_{1} \leqslant m$, где $b_{2}=b_{1}-(m-1) e_{0}$. 
Пусть, наконец, $H \subset G, \rho(G)<\rho(H)<m /(m-1)$. Тогда

$$
\rho(H)=\frac{m(t-1)+b_{1}+e_{0}-y}{(m-1)(t-1)+b_{1}-x}
$$

для некоторых натуральных чисел $x, y$. Справедливо неравенство $y \geqslant x$, так как граф $G$ - связный. Докажем, что

$$
\rho(H)=1+\frac{1}{m-1+b /\left(t+e_{0}-1+x-y\right)}, \quad \text { где } \quad b \leqslant m .
$$

Имеем

$$
\rho(H)=1+\frac{1}{m-1+\left(b_{1}+y(m-1)-m x-(m-1) e_{0}\right) /\left(t-1+e_{0}+x-y\right)} .
$$

Tак как $\rho(H)>\rho(G)$, то

$$
\frac{b_{1}+y(m-1)-m x-(m-1) e_{0}}{t-1+e_{0}+x-y}<\frac{b_{1}-(m-1) e_{0}}{t-1+e_{0}} .
$$

Но знаменатель первой дроби не больше, чем знаменатель второй. Следовательно,

$$
b=b_{1}+y(m-1)-m x-(m-1) e_{0}<b_{1}-(m-1) e_{0} \leqslant m,
$$

что и требовалось доказать.

Перейдем к доказательству свойства 2. В силу определения $m$-расширений первого и второго типа если $G_{0}, G_{1}, \ldots, G_{t}, G$ есть $m$-разложение некоторого графа $G \in \mathscr{H}_{m}$, то $v\left(G_{i+1}\right)-v\left(G_{i}\right) \leqslant m-1, e\left(G_{i+1}\right)-e\left(G_{i}\right)=v\left(G_{i+1}\right)-v\left(G_{i}\right)+1$ для всех $i \in\{0, \ldots, t-1\}$. Следовательно, для каждого $n \in \mathbb{N}$ и для любого графа $G \in \mathscr{H}_{m}$, количество вершин которого не меньше $(m-1) n+1$, справедливо неравенство $\rho(G) \geqslant m n /((m-1) n+1)$. Действительно, плотность любого графа из множества $\mathscr{H}_{m}$ меньше, чем $m /(m-1)$, поэтому при добавлении к нему его $m$-расширения первого или второго типа его плотность увеличивается. Кроме того, для любого $\rho \in(1, m /(m-1))$ найдется такое число $n_{0} \in \mathbb{N}$, что при всех натуральных $n \geqslant n_{0}$ выполнено неравенство $m n /((m-1) n+1)>\rho$. Заметим, наконец, что в любом графе из $\mathscr{H}_{m}$ на более чем $(m-1)(n+1)+1$ вершинах найдется подграф из $\mathscr{H}_{m}$, количество вершин которого находится в отрезке $[(m-1) n+1,(m-1)(n+1)+1]$. Поэтому, очевидно, для $\eta=(m-1)\left(n_{0}+1\right)+1$ утверждение леммы выполнено. Лемма полностью доказана.

Возвращаясь к доказательству теоремы 24, обратимся к описанию стратегии в игре $\operatorname{EHR}\left(G\left(N, N^{-\alpha}\right), G\left(M, M^{-\alpha}\right), k\right)$, являющейся выигрышной для Консерватора с вероятностью, стремящейся к 1. Всюду далее мы считаем, что в каждом раунде игроки выбирают вершины, отличные от уже выбранных. Такое предположение не ограничивает общности в силу того, что размеры графов в рассматриваемых случаях, а также утверждения, используемые в рассуждениях, позволяют выбирать вершины, отличные от уже выбранных. Сначала 
мы опишем некоторые свойства графов $A, B$, благодаря которым Консерватор выигрывает в игре на этих графах, придерживаясь такой стратегии, а затем докажем, что случайный граф обладает этими свойствами с вероятностью, стремящейся к 1 .

Пусть $n_{1}, n_{2}, n_{3}, n_{4}$ - некоторые натуральные числа, $n_{2} \leqslant n_{1}, n_{4} \leqslant n_{3}$, $\rho$ - произвольное положительное число. Будем говорить, что граф $G$ является $\left(n_{1}, n_{2}, n_{3}, n_{4}, \rho\right)$-разреженным, если он обладает следующими свойствами.

1) Пусть $K$ - граф, количество вершин которого не превосходит $n_{1}$. Если $\rho^{\max }(K)<\rho$, то $G$ содержит подграф, изоморфный $K$. Если $\rho^{\max }(K)>\rho$, то $G$ не содержит подграфа, изоморфного $K$.

2) Пусть $\mathscr{H}$ - множество таких $1 / \rho$-надежных пар $\left(H_{1}, H_{2}\right)$, что $v\left(H_{1}\right) \leqslant n_{1}$, $v\left(H_{2}\right) \leqslant n_{2}$. Пусть $\mathscr{K}-$ множество таких $1 / \rho$-жестких пар $\left(K_{1}, K_{2}\right)$, что $v\left(K_{1}\right) \leqslant n_{3}, v\left(K_{2}\right) \leqslant n_{4}$. Тогда для любых пар $\left(H_{1}, H_{2}\right) \in \mathscr{H}$, $\left(K_{1}, K_{2}\right) \in \mathscr{K}$ и для любого подграфа $G_{2} \subset G$ на $v\left(H_{2}\right)$ вершинах в графе $G$ найдется подграф $G_{1}$, являющийся $\left(K_{1}, K_{2}\right)$-максимальным в $G$ точным $\left(H_{1}, H_{2}\right)$-расширением графа $G_{2}$.

Выигрышная стратегия Консерватора, описанная ниже, опирается именно на свойство $\left(n_{1}, n_{2}, n_{3}, n_{4}, \rho\right)$-разреженности обоих графов, на которых играют Новатор и Консерватор (при некоторых значениях $n_{1}, n_{2}, n_{3}, n_{4}, \rho$ ).

Пусть $\rho=1 / \alpha$. Тогда с учетом условий доказываемой нами теоремы имеем

$$
\rho \in\left(1, \frac{2^{k-1}}{2^{k-1}-1}\right), \quad \rho \notin\left\{1+\frac{1}{2^{k-1}-1+b / a}, a, b \in \mathbb{N}, b \leqslant 2^{k-1}\right\} .
$$

Обозначим $\eta(\rho)$ число из формулировки леммы 3 , т. е. такое число, что для любого натурального $v>\eta(\rho)$ у любого графа $G \in \mathscr{H}_{2^{k-1}}$ на $v$ вершинах найдется подграф на не более чем $\eta(\rho)$ вершинах с плотностью, превосходящей $\rho$. Положим

$$
\begin{gathered}
n_{1}(\rho)=\eta(\rho)+k\left(\left[\frac{1}{\rho-1}\right]+1\right), \quad n_{2}(\rho)=\eta(\rho)+(k-2)\left(\left[\frac{1}{\rho-1}\right]+1\right), \\
n_{3}=2^{k-2}+1, \quad n_{4}=2 .
\end{gathered}
$$

Пусть графы $A, B$ являются $\left(n_{1}(\rho), n_{2}(\rho), n_{3}, n_{4}, \rho\right)$-разреженными. Сперва опишем стратегию Консерватора в игре $\operatorname{EHR}(A, B, k)$, а позже докажем, что случайный граф с асимптотической вероятностью 1 является $\left(n_{1}(\rho), n_{2}(\rho), n_{3}\right.$, $\left.n_{4}, \rho\right)$-разреженным. Это и завершит доказательство теоремы.

Итак, начнем со стратегии. Будем обозначать $X_{i}$ граф, выбранный Новатором в $i$-м раунде. Оставшийся граф будем обозначать $Y_{i}$. Вершины, выбранные в графе $X_{i}$ в первых $i$ раундах, обозначим $x_{i}^{1}, \ldots, x_{i}^{i}$, в графе $Y_{i}-$ $y_{i}^{1}, \ldots, y_{i}^{i}$. Итак, пусть в первом раунде Новатор выбрал вершину $x_{1}^{1}$. В силу леммы 3 и свойства $\left(n_{1}(\rho), n_{2}(\rho), n_{3}, n_{4}, \rho\right)$-разреженности графа $X_{1}$ в нем не существует подграфа, изоморфного некоторому графу из $\mathscr{H}_{2^{k-1}}$ с количеством вершин, превосходящим $\eta(\rho)$. Обозначим $\widetilde{X}_{1}^{1}$ подграф в $X_{1}$, изоморфный некоторому графу из $\mathscr{H}_{2^{k-1}}$, содержащий вершину $x_{1}^{1}$ и обладающий следующим свойством максимальности. Если $v_{1}$ - число вершин в $\widetilde{X}_{1}^{1}$, то в $X_{1}$ 
не существует подграфа, содержащего вершину $x_{1}^{1}$ и изоморфного некоторому графу из $\mathscr{H}_{2^{k-1}}$, количество вершин которого превосходит $v_{1}$. Выполнены неравенства $v_{1} \leqslant \eta(\rho)<n_{1}(\rho)$. Поэтому в силу леммы 3 и свойства $\left(n_{1}(\rho), n_{2}(\rho), n_{3}, n_{4}, \rho\right)$-разреженности графа $X_{1}$ плотность графа $\widetilde{X}_{1}^{1}$ меньше, чем $\rho$. Следовательно, по свойству $\left(n_{1}(\rho), n_{2}(\rho), n_{3}, n_{4}, \rho\right)$-разреженности графа $Y_{1}$ в нем найдется подграф $\widetilde{Y}_{1}^{1}$, изоморфный $\widetilde{X}_{1}^{1}$. Пусть при соответствующем изоморфизме $\varphi_{1}: \widetilde{X}_{1}^{1} \rightarrow \widetilde{Y}_{1}^{1}$ вершина $x_{1}^{1}$ переходит в вершину $y_{1}^{1}$, которую и выберет Консерватор в первом раунде.

Пусть сыграно $i$ раундов, $1 \leqslant i<k$. Опишем стратегию Консерватора в $(i+1)$-м раунде. Ниже мы определим ряд свойств подграфов в $A, B$ (они обозначены (I), (II) и (III)). Мы предположим, что в $A$ и $B$ содержатся подграфы, обладающие этими свойствами. В соответствии со свойством (I) выбранные вершины $x_{1}^{i}, \ldots, x_{i}^{i}, y_{1}^{i}, \ldots, y_{i}^{i}$ должны принадлежать объединению этих подграфов. Затем мы докажем, что независимо от выбора Новатором вершины $x_{i+1}^{i+1}$ Консерватор сможет найти такую вершину $y_{i+1}^{i+1}$, что вершины $x_{1}^{i+1}, \ldots, x_{i+1}^{i+1}$, $y_{1}^{i+1}, \ldots, y_{i+1}^{i+1}$ также будут содержаться в подграфах, обладающих свойствами (I), (II) и (III). Кроме того, станет очевидно, что вершины $x_{1}^{1}, y_{1}^{1}$ содержатся в подграфах, обладающих упомянутыми свойствами, откуда по индукции последует аналогичное утверждение для последнего раунда, т. е. для вершин $x_{1}^{k}, \ldots, x_{k}^{k}, y_{1}^{k}, \ldots, y_{k}^{k}$. В частности, из этих свойств мы выведем, что графы $\left.X_{k}\right|_{\left\{x_{1}^{k}, \ldots, x_{k}^{k}\right\}}$ и $\left.Y_{k}\right|_{\left\{y_{1}^{k}, \ldots, y_{k}^{k}\right\}}$ изоморфны.

Воспользуемся обозначением, которое мы ввели в разделе 3 , а именно обозначением $d_{Q}\left(W_{1}, W_{2}\right)$, где $Q$ - некоторый граф, а $W_{1}, W_{2}$ - некоторые его подграфы. Пусть $r$ - произвольное натуральное число, не превосходящее $i$. Пусть, кроме того, $W_{1}, \ldots, W_{r}$ - подграфы в $Q$. Будем говорить, что $W_{1}, \ldots, W_{r}$ обладают $(k, i, r)$-свойством в $Q$, если

- любые два графа из $W_{1}, \ldots, W_{r}$ не имеют общих вершин;

- для любых различных $j_{1}, j_{2} \in\{1, \ldots, r\}$ справедливо неравенство

$$
d_{Q}\left(W_{j_{1}}, W_{j_{2}}\right)>2^{k-i}
$$

- для любого $j \in\{1, \ldots, r\}$ в графе $Q$ не существует подграфа, являющегося $2^{k-i}$-расширением графа $W_{j}$ первого или второго типа;

- мощность множества $V\left(W_{1} \cup \cdots \cup W_{r}\right)$ не превосходит

$$
\eta(\rho)+(i-1)\left(\left[\frac{1}{\rho-1}\right]+1\right) .
$$

Предположим, чтто для некоторого $r \in\{1, \ldots, i\}$ графы $X_{i}$ и $Y_{i}$ содержат подграфы $\widetilde{X}_{i}^{1}, \ldots, \widetilde{X}_{i}^{r}$ и $\widetilde{Y}_{i}^{1}, \ldots, \widetilde{Y}_{i}^{r}$ соответственно, которые обладают следующими свойствами.

(I) Вершины $x_{i}^{1}, \ldots, x_{i}^{i}$ принадлежат множеству $V\left(\widetilde{X}_{i}^{1} \cup \cdots \cup \widetilde{X}_{i}^{r}\right)$, вершины $y_{i}^{1}, \ldots, y_{i}^{i}$ принадлежат множеству $V\left(\tilde{Y}_{i}^{1} \cup \cdots \cup \widetilde{Y}_{i}^{r}\right)$.

(II) Графы $\widetilde{X}_{i}^{1}, \ldots, \tilde{X}_{i}^{r}$ обладают $(k, i, r)$-свойством в $X_{i}$, графы $\tilde{Y}_{i}^{1}, \ldots, \tilde{Y}_{i}^{r}$ обладают $(k, i, r)$-свойством в $Y_{i}$. 
(III) Графы $\widetilde{X}_{i}^{j}$ и $\widetilde{Y}_{i}^{j}$ изоморфны при каждом $j \in\{1, \ldots, r\}$ и при некотором соответствующем изоморфизме (общем для всех графов, так как они не имеют общих вершин) вершины $x_{i}^{j}$ переходят в вершины $y_{i}^{j}, j \in\{1, \ldots, i\}$.

Если $X_{i+1}=X_{i}$, то положим $\tilde{X}_{i+1}^{j}=\tilde{X}_{i}^{j}, \tilde{Y}_{i+1}^{j}=\tilde{Y}_{i}^{j}, j \in\{1, \ldots, r\}$. В противном случае положим $\widetilde{X}_{i+1}^{j}=\widetilde{Y}_{i}^{j}, \widetilde{Y}_{i+1}^{j}=\widetilde{X}_{i}^{j}, j \in\{1, \ldots, r\}$. Пусть $\varphi_{i+1}-$ изоморфизм из $\widetilde{X}_{i+1}^{1} \cup \cdots \cup \widetilde{X}_{i+1}^{r}$ в $\widetilde{Y}_{i+1}^{1} \cup \cdots \cup \widetilde{Y}_{i+1}^{r}$, переводящий графы $\widetilde{X}_{i+1}^{j}$ в $\tilde{Y}_{i+1}^{j}$ при $j \in\{1, \ldots, r\}$. Пусть, кроме того, $\varphi_{i+1}\left(x_{i+1}^{j}\right)=y_{i+1}^{j}$ при $j \in\{1, \ldots, i\}$. Рассмотрим далее три различные ситуации.

1. Предположим, что Новатор в $(i+1)$-м раунде выбрал вершину $x_{i+1}^{i+1}$ из множества $V\left(\widetilde{X}_{i+1}^{1} \cup \cdots \cup \widetilde{X}_{i+1}^{r}\right)$. Тогда Консерватор выберет $y_{i+1}^{i+1}=\varphi\left(x_{i+1}^{i+1}\right)$. Заметим, что мы определили графы $\widetilde{X}_{i+1}^{1}, \ldots, \widetilde{X}_{i+1}^{r}, \widetilde{Y}_{i+1}^{1}, \ldots, \widetilde{Y}_{i+1}^{r}$, просто переобозначив графы $\widetilde{X}_{i}^{1}, \ldots, \widetilde{X}_{i}^{r}, \widetilde{Y}_{i}^{1}, \ldots, \widetilde{Y}_{i}^{r}$ и не меняя их структуры. Поэтому, как нетрудно видеть, при $i<k-1$ графы $\widetilde{X}_{i+1}^{1}, \ldots, \widetilde{X}_{i+1}^{r}$ обладают $(k, i+$ $1, r)$-свойством в $X_{i+1}$, графы $\tilde{Y}_{i+1}^{1}, \ldots, \tilde{Y}_{i+1}^{r}$ обладают $(k, i+1, r)$-свойством в $Y_{i+1}$. Кроме того, графы $\widetilde{X}_{i+1}^{j}$ и $\widetilde{Y}_{i+1}^{j}$ изоморфны при каждом $j \in\{1, \ldots, r\}$ и при соответствующем изоморфизме $\varphi_{i+1}$ (одном и том же для всех графов) вершины $x_{i+1}^{j}$ переходят в вершины $y_{i+1}^{j}, j \in\{1, \ldots, i+1\}$. Иными словами, для $(i+1)$-го раунда мы подобрали графы $\widetilde{X}_{i+1}^{1}, \ldots, \widetilde{X}_{i+1}^{r}, \widetilde{Y}_{i+1}^{1}, \ldots, \widetilde{Y}_{i+1}^{r}$, обладающие свойствами (I), (II) и (III).

2. Предположим теперь, что Новатор выбрал вершину $x_{i+1}^{i+1}$, не принадлежащую множеству $V\left(\widetilde{X}_{i+1}^{1} \cup \cdots \cup \widetilde{X}_{i+1}^{r}\right)$, но при этом

$$
d_{X_{i+1}}\left(x_{i+1}^{i+1}, \widetilde{X}_{i+1}^{1} \cup \cdots \cup \widetilde{X}_{i+1}^{r}\right) \leqslant 2^{k-1-i} .
$$

Заметим, что в силу определения графов $\widetilde{X}_{i+1}^{1}, \ldots, \widetilde{X}_{i+1}^{r}$ в графе $X_{i+1}$ найдется ровно одна цепь $c_{X_{i+1}}$, которая проходит только через вершины графа

$$
X_{i+1} \backslash\left(\tilde{X}_{i+1}^{1} \cup \cdots \cup \tilde{X}_{i+1}^{r}\right)
$$

(не считая последней вершины), имеет длину, не превосходящую $2^{k-1-i}$, и соединяет $x_{i+1}^{i+1}$ с некоторой вершиной $\widetilde{x}_{i+1}^{l}$ графа $\widetilde{X}_{i+1}^{1} \cup \cdots \cup \widetilde{X}_{i+1}^{r}$, где $l \in$ $\{1, \ldots, r\}, \widetilde{x}_{i+1}^{l} \in V\left(\widetilde{X}_{i+1}^{l}\right)$. Следовательно, пара

$$
\left(\widetilde{X}_{i+1}^{1} \cup \cdots \cup \widetilde{X}_{i+1}^{r} \cup c_{X_{i+1}}, \widetilde{X}_{i+1}^{1} \cup \cdots \cup \widetilde{X}_{i+1}^{r}\right)
$$

является $1 / \rho$-надежной. Кроме того,

$$
\left|V\left(\tilde{Y}_{i+1}^{1} \cup \cdots \cup \tilde{Y}_{i+1}^{r}\right)\right|<\eta(\rho)+(i-1)\left(\left[\frac{1}{\rho-1}\right]+1\right) \leqslant \eta(\rho)+(k-2)\left(\left[\frac{1}{\rho-1}\right]+1\right) .
$$

Поэтому в силу свойства $\left(n_{1}(\rho), n_{2}(\rho), n_{3}, n_{4}, \rho\right)$-разреженности графа $Y_{i+1}$ в нем найдется точное $\left(K_{1}, K_{2}\right)$-максимальное $\left(\widetilde{X}_{i+1}^{1} \cup \cdots \cup \widetilde{X}_{i+1}^{r} \cup c_{X_{i+1}}, \widetilde{X}_{i+1}^{1} \cup\right.$ $\left.\cdots \cup \widetilde{X}_{i+1}^{r}\right)$-расширение графа $\tilde{Y}_{i+1}^{1} \cup \cdots \cup \widetilde{Y}_{i+1}^{r}$ для всех таких $1 / \rho$-жестких 
пар $\left(K_{1}, K_{2}\right)$, что $v\left(K_{2}\right)=2, v\left(K_{1}\right) \leqslant 2^{k-1-i}$. Действительно,

$$
\begin{aligned}
\left|V\left(\tilde{X}_{i+1}^{1} \cup \cdots \cup \widetilde{X}_{i+1}^{r} \cup c_{X_{i+1}}\right)\right| & \leqslant \eta(\rho)+(k-2)\left(\left[\frac{1}{\rho-1}\right]+1\right)+2^{k-1-i} \\
& <\eta(\rho)+(k-2)\left(\left[\frac{1}{\rho-1}\right]+1\right)+\frac{1}{\rho-1} \\
& \leqslant \eta(\rho)+(k-1)\left(\left[\frac{1}{\rho-1}\right]+1\right)=n_{1}(\rho) .
\end{aligned}
$$

Иными словами, существуют такая вершина $y_{i+1}^{i+1} \in V\left(Y_{i+1}\right)$, что

$$
d_{Y_{i+1}}\left(y_{i+1}^{i+1}, \tilde{Y}_{i+1}^{l}\right)=d_{X_{i+1}}\left(x_{i+1}^{i+1}, \tilde{X}_{i+1}^{l}\right)
$$

и единственная цепь $c_{Y_{i+1}}$, имеющая длину не больше $2^{k-1-i}$ и соединяющая $y_{i+1}^{i+1}$ с некоторой вершиной $\widetilde{y}_{i+1}^{l}$ графа $\widetilde{Y}_{i+1}^{1} \cup \cdots \cup \widetilde{Y}_{i+1}^{r}, \widetilde{y}_{i+1}^{l} \in V\left(\widetilde{Y}_{i+1}^{l}\right)$. Переопределим графы $\widetilde{X}_{i+1}^{l}, \widetilde{Y}_{i+1}^{l}$ :

$$
\widetilde{X}_{i+1}^{l}:=\tilde{X}_{i+1}^{l} \cup c_{X_{i+1}}, \quad \widetilde{Y}_{i+1}^{l}:=\tilde{Y}_{i+1}^{l} \cup c_{Y_{i+1}} .
$$

Остальные графы $\widetilde{X}_{i+1}^{1}, \ldots, \widetilde{X}_{i+1}^{l-1}, \tilde{X}_{i+1}^{l+1}, \ldots, \widetilde{X}_{i+1}^{r}, \tilde{Y}_{i+1}^{1}, \ldots, \tilde{Y}_{i+1}^{l-1}, \tilde{Y}_{i+1}^{l+1}, \ldots, \widetilde{Y}_{i+1}^{r}$ оставим без изменений. Продолжим изоморфизм $\varphi_{i+1}$ графов на вершины из $V\left(\tilde{X}_{i+1}^{l}\right)$ : для конечной вершины $v$ цепи $c_{X_{i+1}}$, отличной от $\widetilde{x}_{i+1}^{l}$, найдем конечную вершину $u$ цепи $c_{Y_{i+1}}$, отличную от $\widetilde{y}_{i+1}^{l}$, и определим $\varphi_{i+1}(v)=$ $u$. Тогда $\left.\varphi_{i+1}\right|_{\widetilde{X}_{i+1}^{j}}: \widetilde{X}_{i+1}^{j} \rightarrow \widetilde{Y}_{i+1}^{j}-$ изоморфизм при каждом $j \in\{1, \ldots, r\}$ и $\varphi_{i+1}\left(x_{i+1}^{j}\right)=y_{i+1}^{j}$ при всех $j \in\{1, \ldots, i+1\}$, т. е. графы $\tilde{X}_{i+1}^{1}, \ldots, \tilde{X}_{i+1}^{r}$, $\tilde{Y}_{i+1}^{1}, \ldots, \widetilde{Y}_{i+1}^{r}$ обладают свойствами (I) и (III). Докажем, что при $i<k-1$ выполнено свойство (II) (графы $\widetilde{X}_{i+1}^{1}, \ldots, \widetilde{X}_{i+1}^{r}$ обладают $(k, i+1, r)$-свойством в $X_{i+1}$, а графы $\tilde{Y}_{i+1}^{1}, \ldots, \tilde{Y}_{i+1}^{r}$ обладают $(k, i+1, r)$-свойством в $\left.Y_{i+1}\right)$. Для этого достаточно доказать следующие утверждения:

- для любого $j \in\{1, \ldots, r\} \backslash\{l\}$

$$
d_{X_{i+1}}\left(\tilde{X}_{i+1}^{j}, \tilde{X}_{i+1}^{l}\right)>2^{k-i-1}, \quad d_{Y_{i+1}}\left(\tilde{Y}_{i+1}^{j}, \tilde{Y}_{i+1}^{l}\right)>2^{k-i-1}
$$

- в графе $X_{i+1}\left(\right.$ в графе $\left.Y_{i+1}\right)$ не найдется подграфа, являющегося $2^{k-i-1}$ расширением графа $\widetilde{X}_{i+1}^{l}\left(\right.$ графа $\left.\tilde{Y}_{i+1}^{l}\right)$ первого или второго типа;

- мощности множеств $V\left(\widetilde{X}_{i+1}^{1} \cup \cdots \cup \widetilde{X}_{i+1}^{r}\right), V\left(\widetilde{Y}_{i+1}^{1} \cup \cdots \cup \widetilde{Y}_{i+1}^{r}\right)$ не превосходят величины $\eta(\rho)+i([1 /(\rho-1)]+1)$.

Предположим, что найдутся число $j \in\{1, \ldots, r\} \backslash\{l\}$ и цепь, имеющая длину не больше $2^{k-1-i}$ и соединяющая некоторую вершину $u$ графа $\widetilde{X}_{i+1}^{l}$ с некоторой вершиной $v$ графа $\tilde{X}_{i+1}^{j}$. Так как $2^{k-1-i}<2^{k-i}$, то $u \in V\left(c_{X_{i+1}}\right)$. Но длина цепи $c_{X_{i+1}}$ не превосходит $2^{k-1-i}$. Следовательно,

$$
d_{X_{i+1}}\left(\tilde{X}_{i+1}^{j}, \tilde{X}_{i+1}^{l} \backslash\left(c_{X_{i+1}} \backslash\left\{\widetilde{x}_{i+1}^{l}\right\}\right)\right) \leqslant 2^{k-1-i}+2^{k-1-i}=2^{k-i} .
$$


Однако величина в левой части неравенства равна

$$
d_{X_{i}}\left(\tilde{X}_{i}^{l}, \tilde{X}_{i}^{j}\right) \text { либо } \quad d_{Y_{i}}\left(\tilde{Y}_{i}^{l}, \tilde{Y}_{i}^{j}\right) .
$$

Получили противоречие либо с $(k, i, r)$-свойством графов $\widetilde{X}_{i}^{1}, \ldots, \widetilde{X}_{i}^{r}$, либо с $(k, i, r)$-свойством графов $\tilde{Y}_{i}^{1}, \ldots, \widetilde{Y}_{i}^{r}$. Таким образом, $d_{X_{i+1}}\left(\tilde{X}_{i+1}^{j}, \widetilde{X}_{i+1}^{l}\right)>$ $2^{k-i-1}$. Аналогично доказывается неравенство $d_{Y_{i+1}}\left(\tilde{Y}_{i+1}^{j}, \widetilde{Y}_{i+1}^{l}\right)>2^{k-i-1}$.

Доказательство второго утверждения мы тоже приводим только для графа $X_{i+1}$, так как оно в точности повторяет доказательство для графа $Y_{i+1}$. Итак, пусть в графе $X_{i+1}$ существует подграф $W$, являющийся $2^{k-1-i}$-расширением первого или второго типа графа $\widetilde{X}_{i+1}^{l}$. Рассмотрим множество ребер

$$
E=E(W) \backslash\left(E\left(\widetilde{X}_{i+1}^{l}\right) \cup E\left(W \backslash \widetilde{X}_{i+1}^{l}\right)\right) .
$$

Вершин, принадлежащих множеству $V\left(\widetilde{X}_{i+1}^{l}\right)$ и являющихся концами ребер из $E$, не более двух. Обозначим их $v_{1}$ и $v_{2}$ (вообще говоря, эти вершины могут совпадать). Если

$$
v_{1}, v_{2} \in V\left(\widetilde{X}_{i+1}^{l}\right) \backslash\left(V\left(c_{X_{i+1}}\right) \backslash\left\{\widetilde{x}_{i+1}^{l}\right\}\right),
$$

то мы приходим к противоречию либо с $(k, i, r)$-свойством графов $\widetilde{X}_{i}^{1}, \ldots, \widetilde{X}_{i}^{r}$, либо с $(k, i, r)$-свойством графов $\tilde{Y}_{i}^{1}, \ldots, \widetilde{Y}_{i}^{r}$. Если же хотя бы одна из вершин $v_{1}, v_{2}$ не принадлежит множеству

$$
V\left(\tilde{X}_{i+1}^{l}\right) \backslash\left(V\left(c_{X_{i+1}}\right) \backslash\left\{\widetilde{x}_{i+1}^{l}\right\}\right),
$$

то в графе $W$ найдется подграф $W_{1}$, множество вершин которого содержит

$$
V\left(\widetilde{X}_{i+1}^{l}\right) \backslash\left(V\left(c_{X_{i+1}}\right) \backslash\left\{\widetilde{x}_{i+1}^{l}\right\}\right)
$$

и который является $2^{k-i}$-расширением графа

$$
\left.W\right|_{V\left(\widetilde{X}_{i+1}^{l}\right) \backslash\left(V\left(c_{X_{i+1}}\right) \backslash\left\{\widetilde{x}_{i+1}^{l}\right\}\right)}
$$

первого или второго типа. Мы снова приходим к противоречию либо с $(k, i, r)$ свойством графов $\widetilde{X}_{i}^{1}, \ldots, \widetilde{X}_{i}^{r}$, либо с $(k, i, r)$-свойством графов $\widetilde{Y}_{i}^{1}, \ldots, \widetilde{Y}_{i}^{r}$.

Последнее утверждение выполнено, так как количество добавленных вершин не превосходит $2^{k-1-i} \leqslant[1 /(\rho-1)]+1$.

Если $i=k-1$, то в обоих случаях $\varphi_{k}: \tilde{X}_{k}^{1} \cup \cdots \cup \widetilde{X}_{k}^{r} \rightarrow \widetilde{Y}_{k}^{1} \cup \cdots \cup \tilde{Y}_{k}^{r}-$ изоморфизм и $\varphi_{k}\left(x_{k}^{j}\right)=y_{k}^{j}$ при всех $j \in\{1, \ldots, k\}$, а стало быть, графы $\left.X_{k}\right|_{\left\{x_{k}^{1}, \ldots, x_{k}^{k}\right\}}$, $\left.Y_{k}\right|_{\left\{y_{k}^{1}, \ldots, y_{k}^{k}\right\}}$ также изоморфны и Консерватор побеждает.

3. Пусть, наконец,

$$
d_{X_{i+1}}\left(x_{i+1}^{i+1}, \tilde{X}_{i+1}^{1} \cup \cdots \cup \widetilde{X}_{i+1}^{r}\right)>2^{k-1-i} .
$$

Найдем подграф в $X_{i+1}$, содержащий наибольшее количество вершин, одна из которых совпадает с $x_{i+1}^{i+1}$, и изоморфный некоторому графу из множест- 
ва $\mathscr{H}_{2^{k-1-i}}$. Обозначим полученный граф $\tilde{X}_{i+1}^{r+1}$. В силу свойства $\left(n_{1}(\rho), n_{2}(\rho)\right.$, $\left.n_{3}, n_{4}, \rho\right)$-разреженности графа $X_{i+1}$ он содержит не более одного простого цикла.

Рассмотрим пару $\left(H_{1}, H_{2}\right)$, где граф $H_{1}$ является цепью длины $[1 /(\rho-1)]+1$, объединенной с графом, изоморфным $\widetilde{X}_{i+1}^{r+1}$ (вершина $x_{i+1}^{i+1}$ при соответствующем изоморфизме переходит в некоторую вершину $h)$. Граф $H_{2}$ содержит лишь одну вершину, которая является концевой вершиной рассмотренной цепи, отличной от $h$. Рассмотрим, кроме того, множество $\mathscr{K}$ всех попарно неизоморфных пар $\left(K_{1}, K_{2}\right)$, для каждой из которых найдется такой граф $K$, что $K \cap K_{1}=K_{2}$, граф $K \cup K_{1}$ является $2^{k-1-i}$-расширением графа $K$ первого или второго типа. В силу $\left(n_{1}(\rho), n_{2}(\rho), n_{3}, n_{4}, \rho\right)$-разреженности графа $Y_{i+1}$ найдутся такие вершины

$$
y_{i+1}^{i+1} \in V\left(Y_{i+1}\right) \backslash V\left(\tilde{Y}_{i+1}^{1} \cup \cdots \cup \tilde{Y}_{i+1}^{r}\right), \quad \widetilde{y}_{i+1}^{l} \in V\left(\tilde{Y}_{i+1}^{l}\right)
$$

для некоторого $l \in\{1, \ldots, r\}$, такая цепь $c_{Y_{i+1}} \subset Y_{i+1}$ длины $[1 /(\rho-1)]+1$, соединяющая вершины $\widetilde{y}_{i+1}^{l}$ и $y_{i+1}^{i+1}$, а также такой граф $\widetilde{Y}_{i+1}^{r+1} \subset Y_{i+1}$, изоморфный $\tilde{X}_{i+1}^{r+1}$, что

$$
d_{Y_{i+1}}\left(y_{i+1}^{i+1}, \tilde{Y}_{i+1}^{1} \cup \cdots \cup \tilde{Y}_{i+1}^{r}\right)=\left[\frac{1}{\rho-1}\right]+1, \quad V\left(c_{Y_{i+1}}\right) \cap V\left(\widetilde{Y}_{i+1}^{r+1}\right)=\left\{y_{i+1}^{i+1}\right\}
$$

и пара

$$
\left(c_{Y_{i+1}} \cup \tilde{Y}_{i+1}^{r+1},\left(\left\{\widetilde{y}_{i+1}^{l}\right\}, \varnothing\right)\right)
$$

является $\left(K_{1}, K_{2}\right)$-максимальным в $Y_{i+1}$ точным $\left(H_{1}, H_{2}\right)$-расширением графа $\left(\left\{\widetilde{y}_{i+1}^{l}\right\}, \varnothing\right)$ для всех $\left(K_{1}, K_{2}\right) \in \mathscr{K}$.

Продолжим изоморфизм графов $\varphi_{i+1}$ на вершины из множества $V\left(\widetilde{X}_{i+1}^{r+1}\right)$ : $\left.\varphi_{i+1}\right|_{\tilde{X}_{i+1}^{r+1}}: \widetilde{X}_{i+1}^{r+1} \rightarrow \tilde{Y}_{i+1}^{r+1}$, причем $\varphi_{i+1}\left(x_{i+1}^{i+1}\right)=y_{i+1}^{i+1}$.

Докажем, что графы $\widetilde{X}_{i+1}^{1}, \ldots, \widetilde{X}_{i+1}^{r+1}, \widetilde{Y}_{i+1}^{1}, \ldots, \widetilde{Y}_{i+1}^{r+1}$ обладают свойствами (I), (II) и (III) при $i<k-1$. Свойство (I) выполнено, так как $x_{i+1}^{i+1} \in V\left(\widetilde{X}_{i+1}^{r+1}\right)$, $y_{i+1}^{i+1} \in V\left(\widetilde{Y}_{i+1}^{r+1}\right)$. Из изоморфности пар

$$
\left(c_{Y_{i+1}} \cup \tilde{Y}_{i+1}^{r+1},\left(\left\{\widetilde{y}_{i+1}^{l}\right\}, \varnothing\right)\right) \quad \text { и } \quad\left(H_{1}, H_{2}\right)
$$

следует изоморфность пар

$$
\left(\widetilde{X}_{i+1}^{1} \cup \cdots \cup \widetilde{X}_{i+1}^{r+1}, \widetilde{X}_{i+1}^{1} \cup \cdots \cup \widetilde{X}_{i+1}^{r}\right) \quad \text { и } \quad\left(\widetilde{Y}_{i+1}^{1} \cup \cdots \cup \tilde{Y}_{i+1}^{r+1}, \widetilde{Y}_{i+1}^{1} \cup \cdots \cup \widetilde{Y}_{i+1}^{r}\right)
$$

и справедливость свойства (III). Осталось доказать, что графы $\widetilde{X}_{i+1}^{1}, \ldots, \widetilde{X}_{i+1}^{r+1}$ обладают $(k, i+1, r+1)$-свойством в $X_{i+1}$, а графы $\widetilde{Y}_{i+1}^{1}, \ldots, \tilde{Y}_{i+1}^{r+1}$ обладают $(k, i+1, r+1)$-свойством в $Y_{i+1}$. Пусть $j \in\{1, \ldots, r\}$. Предположим, что существует такая вершина $v \in V\left(\widetilde{X}_{i+1}^{r+1}\right)$, отличная от $x_{i+1}^{i+1}$, что $d_{X_{i+1}}\left(v, \widetilde{X}_{i+1}^{j}\right)<$ $2^{k-1-i}$. Тогда в графе $X_{i+1}$ существует подграф, являющийся $2^{k-i}$-расширением графа $\widetilde{X}_{i+1}^{j}$ первого или второго типа. Получили противоречие с $(k, i, r)$ свойством графов $\widetilde{X}_{i+1}^{1}, \ldots, \widetilde{X}_{i+1}^{r}$ в $X_{i+1}$. Следовательно, $d_{X_{i+1}}\left(\widetilde{X}_{i+1}^{j}, \tilde{X}_{i+1}^{r+1}\right) \geqslant$ 
$2^{k-1-i}$. Кроме того,

$$
\begin{aligned}
d_{Y_{i+1}}\left(\tilde{Y}_{i+1}^{j}, \tilde{Y}_{i+1}^{r+1}\right) & >d_{Y_{i+1}}\left(\tilde{Y}_{i+1}^{j}, y_{i+1}^{i+1}\right)-\left|V\left(\tilde{Y}_{i+1}^{r+1}\right)\right| \\
& \geqslant\left[\frac{1}{\rho-1}\right]+1-2^{k-1-i}>2^{k-1}-2^{k-1-i} \geqslant 2^{k-2} \geqslant 2^{k-1-i}
\end{aligned}
$$

Граф $\widetilde{X}_{i+1}^{r+1}\left(\right.$ граф $\left.\tilde{Y}_{i+1}^{r+1}\right)$ не имеет общих вершин с графом $\widetilde{X}_{i+1}^{1} \cup \cdots \cup \widetilde{X}_{i+1}^{r}$ (графом $\widetilde{Y}_{i+1}^{1} \cup \cdots \cup \widetilde{Y}_{i+1}^{r}$ ) по построению. Справедливы соотношения

$$
\begin{aligned}
\left|V\left(W_{1} \cup \cdots \cup W_{r+1}\right)\right| & =\left|V\left(W_{1} \cup \cdots \cup W_{r}\right)\right|+\left|V\left(W_{r+1}\right)\right| \\
& \leqslant \eta(\rho)+(i-1)\left(\left[\frac{1}{\rho-1}\right]+1\right)+2^{k-1-i} \\
& <\eta(\rho)+i\left(\left[\frac{1}{\rho-1}\right]+1\right)
\end{aligned}
$$

где либо $W_{j}=\tilde{X}_{i+1}^{j}$ для всех $j \in\{1, \ldots, r+1\}$, либо $W_{j}=\tilde{Y}_{i+1}^{j}$ для всех $j \in\{1, \ldots, r+1\}$. Докажем, наконец, что в графе $X_{i+1}$ (в графе $\left.Y_{i+1}\right)$ не найдется подграфа, являющегося $2^{k-1-i}$-расширением графа $\tilde{X}_{i+1}^{r+1}\left(\right.$ графа $\tilde{Y}_{i+1}^{r+1}$ ). В случае графа $\tilde{X}_{i+1}^{r+1}$ достаточно вспомнить, что он содержит наибольшее количество вершин среди всех графов, изоморфных какому-либо графу из $\mathscr{H}_{2^{k-1-i}}$ и содержащих вершину $x_{i+1}^{i+1}$. Граф $Y_{i+1}$ не содержит графов, являющихся $2^{k-1-i}$-расширениями графа $\tilde{Y}_{i+1}^{r+1}$ первого или второго типа, так как граф $\tilde{Y}_{i+1}^{r+1}$ является $\left(K_{1}, K_{2}\right)$-максимальным в $Y_{i+1}$ для всех $\left(K_{1}, K_{2}\right) \in \mathscr{K}$.

Если $i=k-1$, то $\varphi_{k}: \widetilde{X}_{k}^{1} \cup \cdots \cup \widetilde{X}_{k}^{r+1} \rightarrow \widetilde{Y}_{k}^{1} \cup \cdots \cup \widetilde{Y}_{k}^{r+1}-$ изоморфизм и $\varphi_{k}\left(x_{k}^{j}\right)=y_{k}^{j}$ при всех $j \in\{1, \ldots, k\}$, а стало быть, графы $\left.X_{k}\right|_{\left\{x_{k}^{1}, \ldots, x_{k}^{k}\right\}}$, $\left.Y_{k}\right|_{\left\{y_{k}^{1}, \ldots, y_{k}^{k}\right\}}$ также изоморфны и Консерватор побеждает.

Напомним, что $\beta \in(0, \infty) \backslash \mathscr{Q}$ - произвольное число, $\alpha=1-1 /\left(2^{k-1}+\beta\right)$, $\rho=1 / \alpha$. Для завершения доказательства нашей теоремы нам остается убедиться в том, что с вероятностью, стремящейся к 1, случайный граф $G\left(N, N^{-\alpha}\right)$ является $\left(n_{1}, n_{2}, n_{3}, n_{4}, \rho\right)$-разреженным, где числа $n_{1}, n_{2}, n_{3}, n_{4}$ определены равенствами (6), (7).

Обратимся сначала к первому свойству в определении $\left(n_{1}, n_{2}, n_{3}, n_{4}, \rho\right)$-разреженного графа. Рассмотрим такое множество $\mathscr{G}$ попарно неизоморфных графов, количество вершин которых не превосходит $n_{1}$, а максимальная плотность отлична от $\rho$, что любой граф $G$ с $v(G) \leqslant n_{1}$ и $\rho^{\max }(G) \neq \rho$ изоморфен некоторому графу из $\mathscr{G}$. Пусть $\mathscr{G}_{1}$ - такое множество попарно неизоморфных графов, количество вершин которых не превосходит $n_{1}$, а максимальная плотность меньше, чем $\rho$, что любой граф, удовлетворяющий заданным условиям, изоморфен некоторому графу из $\mathscr{G}_{1}$. Очевидно, что $\left|\mathscr{G}_{1}\right| \leqslant|\mathscr{G}|<\infty$. Поэтому в соответствии с теоремой 3 справедливы равенства

$$
\begin{array}{r}
\lim _{N \rightarrow \infty} \mathrm{P}_{N, p}\left(\forall G \in \mathscr{G}_{1} N_{G}>0\right)=1, \\
\lim _{N \rightarrow \infty} \mathrm{P}_{N, p}\left(\exists G \in \mathscr{G} \backslash \mathscr{G}_{1} N_{G}>0\right)=0 .
\end{array}
$$

Свойство 1) доказано. 
Пусть $\mathscr{H}$ - множество таких попарно неизоморфных $1 / \rho$-надежных пар $\left(H_{1}, H_{2}\right)$, что $v\left(H_{1}\right) \leqslant n_{1}, v\left(H_{2}\right) \leqslant n_{2}$ и мощность $\mathscr{H}$ максимальна. Пусть $\mathscr{K}$ - множество таких попарно неизоморфных $1 / \rho$-жестких пар $\left(K_{1}, K_{2}\right)$, что $v\left(K_{1}\right) \leqslant n_{3}, v\left(K_{2}\right) \leqslant n_{4}$ и мощность $\mathscr{K}$ максимальна. В силу теоремы 13 с вероятностью, стремящейся к 1 , выполнено

$$
\begin{gathered}
\forall \widetilde{x}_{1}, \ldots, \widetilde{x}_{v\left(H_{2}\right)} \in V_{N} \quad \forall\left(H_{1}, H_{2}\right) \in \mathscr{H} \quad \forall\left(K_{1}, K_{2}\right) \in \mathscr{K} \\
\left(N_{\left(H_{1}, H_{2}\right)}^{\left(K_{1}, K_{2}\right)}\left(\widetilde{x}_{1}, \ldots, \widetilde{x}_{v\left(H_{2}\right)}\right)>0\right) .
\end{gathered}
$$

Тем самым, свойство 2), а вместе с ним и теорема 24 доказаны.

В теореме 22 и теореме 24 утверждается справедливость $k$-закона нуля или единицы при некоторых рациональных $\alpha$ из $(0,1)$. Что можно сказать о других значениях $\alpha$ из этого интервала? Справедлив ли закон нуля или единицы на концах интервалов, рассмотренных в этих теоремах? Частично мы ответили на этот вопрос в работах [60], [62], [63].

Теорема 25. Пусть $k \geqslant 3$ - произвольное натуральное число. Случайный граф $G\left(N, N^{-1 /(k-2)}\right)$ не подчиняется $k$-закону нуля или единицы. Пусть теперь $k>3$ и $\widetilde{\mathscr{Q}}$ - множество натуральных чисел, не превосходящих $2^{k-1}-2$. Случайный граф $G\left(N, N^{-\alpha}\right)$ не подчиняется $k$-закону нуля или единицы, если $\alpha=1-1 /\left(2^{k-1}+\beta\right), \beta \in \widetilde{\mathscr{Q}}$. Если же $\alpha \in\left\{1-1 /\left(2^{k-1}-1\right), 1-1 / 2^{k}\right\}$, то случайный граф $G\left(N, N^{-\alpha}\right)$ подчиняется $k$-закону нуля или единиць.

ДокАЗАТЕЛЬСтво этого утверждения громоздкое и довольно техническое, поэтому мы рассмотрим только случай $\alpha=1-1 /\left(2^{k-1}+\beta\right)$, где $\beta$ - некоторое число из $\widetilde{\mathscr{Q}}$.

Для доказательства мы рассмотрим два графа $A$ и $B$, а также свойство $L_{1}$, которым обладает граф $A$, и свойство $L_{2}$, которым обладает граф $B$. Мы докажем, что у Новатора есть выигрышная стратегия в игре $\operatorname{EHR}(A, B, k)$, а затем установим, что случайный граф $G\left(N, N^{-\alpha}\right)$ с вероятностью, стремящейся к некоторому положительному числу, обладает свойствами $L_{1}$ и $L_{2}$. Тем самым в силу теоремы 21 утверждение будет доказано.

Итак, пусть $k>3$ - некоторое натуральное число, $\beta \in \widetilde{Q}, \alpha=1-1 /\left(2^{k-1}+\beta\right)$. Положим

$$
\rho=\frac{1}{\alpha}=\frac{2^{k-1}+\beta}{2^{k-1}+\beta-1} .
$$

Пусть $\beta$ - нечетное число. Тогда рассмотрим граф $X$, равный объединению двух простых циклов $C_{1}, C_{2}$, имеющих ровно одну общую вершину, причем

$$
\begin{aligned}
v\left(C_{1}\right)+v\left(C_{2}\right) & =2^{k-1}+\beta, & & v\left(C_{1}\right) \leqslant 2^{k-1}-1, \\
v\left(C_{2}\right) & \leqslant 2^{k-1}-2, & & v\left(C_{1}\right)>v\left(C_{2}\right),
\end{aligned}
$$

графы $C_{1}$ и $C_{2}$ не имеют общих ребер. Заметим, что $v\left(C_{1}\right)$ и $v\left(C_{2}\right)$ - числа различной четности. Обозначим $L_{1}$ свойство графа содержать подграф, изоморфный $X$. Рассмотрим свойство содержать такую вершину $x$, что в графе существуют различные $v\left(C_{1}\right)$-расширение первого типа подграфа $(\{x\}, \varnothing)$ 
и $v\left(C_{2}\right)$-расширение первого типа того же подграфа. Обозначим отрицание этого свойства $L_{2}$. Пусть граф $A$ обладает свойством $L_{1}$, а граф $B$ обладает свойством $L_{2}$. В первом раунде Новатор выбирает такую вершину $x_{1}$ графа $A$, что $x_{1}$ является вершиной некоторого подграфа $A_{1} \cup A_{2}$ в $A$, изоморфного $X$, причем $A_{1} \cong C_{1}, A_{2} \cong C_{2}, V\left(A_{1}\right) \cap V\left(A_{2}\right)=\left\{x_{1}\right\}$.

Пусть теперь $\beta$ - четное число. Рассмотрим граф $X$, равный объединению двух простых непересекающихся по вершинам циклов $C_{1}, C_{2}$, а также цепи $D$ длины 1 , соединяющей некоторые вершины $c_{1} \in V\left(C_{1}\right), c_{2} \in V\left(C_{2}\right)$, причем

$$
\begin{aligned}
v\left(C_{1}\right)+v\left(C_{2}\right) & =2^{k-1}+\beta-1, & & v\left(C_{1}\right) \leqslant 2^{k-1}-1, \\
v\left(C_{2}\right) & \leqslant 2^{k-1}-2, & & v\left(C_{1}\right)>v\left(C_{2}\right) .
\end{aligned}
$$

Снова заметим, что $v\left(C_{1}\right)$ и $v\left(C_{2}\right)$ - числа различной четности. Обозначим $L_{1}$ свойство графа содержать подграф, изоморфный $X$. Рассмотрим свойство содержать такую вершину $x$, что в графе существуют различные $v\left(C_{1}\right)$-расширение первого типа подграфа $(\{x\}, \varnothing)$ и $\left(v\left(C_{2}\right)+1\right)$-расширение первого типа того же подграфа. Обозначим отрицание этого свойства $L_{2}$. Пусть граф $A$ обладает свойством $L_{1}$, а граф $B$ обладает свойством $L_{2}$. В первом раунде Новатор выбирает такую вершину $x_{1}$ графа $A$, что $x_{1}$ является вершиной некоторого подграфа $A_{1} \cup A_{2}$ в $A$, изоморфного $X$, причем $A_{1} \cong C_{1}, A_{2} \cong C_{2} \cup D$, $V\left(A_{1}\right) \cap V\left(A_{2}\right)=\left\{x_{1}\right\}$.

Консерватор выбирает некоторую вершину $y_{1} \in V(B)$. Очевидно, что в графе $A$ найдутся такие вершины $x_{2}^{1}, x_{2}^{2}$, что граф $A_{1}$ является объединением двух цепей $K_{A}^{1}, T_{A}^{1}$ различной длины, соединяющих вершины $x_{1}$ и $x_{2}^{1}$, а граф $A_{2}$ является объединением двух цепей $K_{A}^{2}, T_{A}^{2}$ различной длины, соединяющих вершины $x_{1}$ и $x_{2}^{2}$. Заметим, что $v\left(K_{A}^{1}\right)+v\left(T_{A}^{1}\right)$ и $v\left(K_{A}^{2}\right)+v\left(T_{A}^{2}\right)$ - числа разной четности. Пусть в графе $B$ существует вершина $y$, соединенная с $y_{1}$ различными цепями $K_{B}^{1}, T_{B}^{1}$, при этом, скажем, $v\left(K_{B}^{1}\right)=v\left(K_{A}^{1}\right), v\left(T_{B}^{1}\right)=v\left(T_{A}^{1}\right)$. Тогда, очевидно, для любой другой вершины, для которой существуют две такие различные цепи $K_{B}^{2}, T_{B}^{2}$, соединяющие ее с $y_{1}$, что $v\left(K_{B}^{2}\right) \leqslant v\left(K_{A}^{2}\right), v\left(T_{B}^{2}\right) \leqslant v\left(T_{A}^{2}\right)$, числа $v\left(K_{B}^{1}\right)+v\left(T_{B}^{1}\right)$ и $v\left(K_{B}^{2}\right)+v\left(T_{B}^{2}\right)$ должны иметь одинаковую четность. Следовательно, в графе $B$ не найдется вершины $y$, соединенной с $y_{1}$ такими различными цепями $K_{B}^{2}, T_{B}^{2}$, что $v\left(K_{B}^{2}\right)=v\left(K_{A}^{2}\right), v\left(T_{B}^{2}\right)=v\left(T_{A}^{2}\right)$. Тогда во втором раунде Новатор выберет вершину $x_{2}=x_{2}^{2}$. Консерватор во втором раунде выбирает некоторую вершину $y_{2} \in V(B)$. Пусть, например, не нашлось цепи длины $v\left(K_{A}^{2}\right)-1$, соединяющей $y_{1}$ с $y_{2}$ в графе $B$. Положим $S_{1}=K_{A}^{2}$. Пусть сыграно $i$ раундов, $i \geqslant 2$. Пусть, кроме того, выбраны вершины $x_{1}, \ldots, x_{i} \in V(A), y_{1}, \ldots, y_{i} \in V(B)$, а также такая цепь $S_{i-1}$, соединяющая вершину $x_{i}$ с некоторой вершиной $x_{j(i)}, j(i) \leqslant i-1$, в графе $A$, что в графе $B$ не найдется цепи такой же длины, соединяющей вершины $y_{i}$ и $y_{j(i)}$. Новатор в $(i+1)$-м раунде выбирает такую вершину $x_{i+1}$, принадлежащую цепи $S_{i-1}$, что величина

$$
\left|d_{S_{i-1}}\left(x_{i}, x_{i+1}\right)-d_{S_{i-1}}\left(x_{j(i)}, x_{i+1}\right)\right|
$$

минимальна. Консерватор выбирает некоторую вершину $y_{i+1} \in V(B)$. Заметим, что в графе $B$ не найдется двух цепей, из которых одна имеет длину 
$d_{S_{i-1}}\left(x_{j(i)}, x_{i+1}\right)$ и соединяет $y_{j(i)}$ с $y_{i+1}$, а вторая имеет длину $d_{S_{i-1}}\left(x_{i}, x_{i+1}\right)$ и соединяет $y_{i}$ с $y_{i+1}$. Пусть, например, не нашлось цепи, соединяющей $y_{j}(i)$ с $y_{i+1}$. Обозначим $S_{i}$ цепь, являющуюся подграфом в $S_{i-1}$ и соединяющую $x_{j(i)}$ с $x_{i+1}$. Так как $\max \left\{v\left(C_{1}\right), v\left(C_{2}\right)\right\} \leqslant 2^{k-1}$, то в одном из раундов с номером $r \in\{3, \ldots, k\}$ Новатор выберет такую вершину $x_{r}$, что она будет соединена ребрами как с вершиной $x_{r-1}$, так и с некоторой вершиной $x_{j(r)}, j(r)<r-1$. В графе $B$ вершины, соединенной и с $y_{r-1}$, и с $y_{j(r)}$, не найдется. Поэтому Новатор победит.

Докажем теперь, что случайный граф $G\left(N, N^{-\alpha}\right)$ с вероятностью, стремящейся к некоторому положительному числу, обладает свойствами $L_{1}$ и $L_{2}$. Граф $X$ строго сбалансированный, а его плотность равна $\rho$. Поэтому в силу теоремы 4 вероятность того, что случайный граф обладает свойством $L_{1}$, стремится $\mathrm{K} 1-e^{-1 / a(X)}$. Для завершения доказательства осталось установить справедливость следующего соотношения: $\lim _{N \rightarrow \infty} \mathrm{P}_{N, N^{-\alpha}}\left(\bar{L}_{2}\right) \in(0,1)$. Легко заметить, что свойство $\bar{L}_{2}$ выполнено тогда и только тогда, когда граф содержит подграф, выбранный из некоторого конечного множества, причем плотность графов из этого множества не меньше, чем $\rho$ (а плотность некоторых равна $\rho$ ). В соответствии с теоремой о совместном распределении чисел подграфов в случайном графе (см. [10; гл. III, замечание 3.20]) с вероятностью, стремящейся к некоторому числу из интервала $(0,1)$, случайный граф $G\left(N, N^{-\alpha}\right)$ содержит хотя бы один подграф из упомянутого множества. Тем самым, теорема доказана.

Будем говорить, что рациональное число $\alpha \in(0,1)$ является $k$-критической точкой, если случайный граф $G\left(N, N^{-\alpha}\right)$ не подчиняется $k$-закону нуля или единицы. Из теорем 22, 24 и 25 следует, что наименьший элемент в множестве $k$-критических точек, которое мы обозначим $S_{k}$, равен $1 /(k-2)$, а наибольший $1-1 /\left(2^{k-1}-2\right)$. Известно, кроме того, что множество $k$-критических точек является бесконечным при достаточно больших $k$. Это следует из утверждения, которое мы приводим ниже.

Пусть $G$ - произвольный граф, а $x_{1}, x_{2}, x_{3}$ - его вершины. Количество вершин, соединенных ребрами в графе $G$ с каждой из вершин $x_{1}, x_{2}, x_{3}$, обозначим $N_{G}\left(x_{1}, x_{2}, x_{3}\right)$. Рассмотрим свойство $L$, выражаемое формулой второго порядка, определенное следующим образом. Граф $G$ обладает свойством $L$ тогда и только тогда, когда число $\max _{x_{1}, x_{2}, x_{3} \in V(G)} N_{G}\left(x_{1}, x_{2}, x_{3}\right)$ четно (максимум берется по всем тройкам вершин, элементы которых попарно различны).

Лемма 4 (Дж. Спенсер, 1990, [64]). Пусть $\delta>0$ - произвольное число, $\alpha=1 / 3+\delta, p=N^{-\alpha}$. Тогда существует такое свойство первого порядка $\widetilde{L}$, чmo $\mathrm{P}_{N, p}(\widetilde{L} \triangle L) \rightarrow 0, N \rightarrow \infty$.

Докажем, что из этой леммы следует бесконечность множества $k$-критических точек при достаточно больших $k$. Пусть $k$ - кванторная глубина формулы, выражающей свойство $\widetilde{L}$, существование которого утверждает лемма. Для доказательства того, что $\left|S_{k}\right|=\infty$, достаточно доказать, что существует бесконечно много таких рациональных $\delta \in(0,2 / 3)$, что случайный граф $G\left(N, N^{-1 / 3-\delta}\right)$ обладает свойством $L$ с асимптотической вероятностью, отличной от 0 и 1 . 
Рассмотрим последовательность $\delta_{n}=1 /(2 n)$. Докажем, что при каждом $n \in \mathbb{N}$ случайный граф $G\left(N, N^{-1 / 3-\delta_{n}}\right)$ обладает свойством $L$ с асимптотической вероятностью, отличной от 0 и 1 . Легко доказать, что для любого $m \in \mathbb{N}$ полный двудольный граф $K_{3, m}$ является строго сбалансированным. Из теоремы 4 следует, что случайный граф $G\left(N, N^{-1 / 3-\delta_{n}}\right)$ содержит $K_{3,2 n}$ с вероятностью, стремящейся к некоторому числу $\tau$, отличному от 0 и 1 . Более того, из теоремы 2 следует, что случайный граф $G\left(N, N^{-1 / 3-\delta_{n}}\right)$ содержит $K_{3,2 n-1}$ и не содержит $K_{3,2 n+1}$ с вероятностью, стремящейся к 1. Следовательно,

$$
\lim _{N \rightarrow \infty} \mathrm{P}_{N, N^{-1 / 3-\delta_{n}}}(L)=\tau \in(0,1),
$$

что завершает доказательство бесконечности множества $S_{k}$.

Обратимся теперь к объекту, который в определенном смысле является более естественным, чем множество $k$-критических точек. Такой объект (мы обозначим его $\widetilde{S}_{k}$ ) был в 1988 г. рассмотрен Дж. Спенсером и С. Шела в [50] и назван спектром. Спектр - это множество всех рациональных $\alpha \in(0,1)$, которые не обладают следующим свойством. Для любого свойства первого порядка $L$, выражаемого формулой, кванторная глубина которой ограничена числом $k$, существует такое число $\varepsilon_{0}>0$, что либо для любого $\varepsilon \in\left(-\varepsilon_{0}, \varepsilon_{0}\right)$ выполнено равенство $\lim _{N \rightarrow \infty} \mathrm{P}_{N, N^{-\alpha+\varepsilon}}(L)=0$, либо для любого $\varepsilon \in\left(-\varepsilon_{0}, \varepsilon_{0}\right)$ выполнено равенство $\lim _{N \rightarrow \infty} \mathrm{P}_{N, N^{-\alpha+\varepsilon}}(L)=1$.

Рассмотрение такого множества мотивировано существованием монотонных свойств первого порядка, пороговые функции которых имеют вид $N^{-\alpha+o(1)}$, где $\alpha \in(0,1)$ - рациональное число. В этой связи при достаточно больших $k$ и при некоторых рациональных $\alpha$ выполнен $k$-закон нуля или единицы для случайного графа $G\left(N, N^{-\alpha}\right)$, тогда как случайный граф $G\left(N, N^{-\alpha+o(1)}\right)$ не подчиняется $k$-закону нуля или единицы. Поэтому естественно находить асимптотические вероятности выполнения свойств первого порядка не только в самой точке, но и в некоторой ее малой окрестности.

Очевидно, что для любого $k \in \mathbb{N}$ выполнено $S_{k} \subseteq \widetilde{S}_{k}$. Кроме того, при достаточно больших $k$ эти множества различны. Следовательно, множество $\widetilde{S}_{k}$ также является бесконечным. Но совпадают ли наименьшие и наибольшие элементы этих множеств? Частичный ответ на этот вопрос дает следующая теорема.

Теорема 26. Выполнено равенство $\widetilde{S}_{3}=\{1 / 2,2 / 3\}$. Если $k>3$, то три наименъших элемента спектра $\widetilde{S}_{k}$ равны

$$
\frac{1}{k-1}, \quad \frac{2}{2 k-3}, \quad \frac{1}{k-2} .
$$

ДокАзАтельство. Докажем сначала, что при любом $k \geqslant 3$ числа $1 /(k-1)$, $2 /(2 k-3)$ принадлежат $\widetilde{S}_{k}$. Рассмотрим два свойства $L_{1}, L_{2}$, которые выражаются с помощью двух формул первого порядка

$$
\begin{aligned}
\phi_{1} & =\forall x_{1} \ldots \forall x_{k-1} \exists x_{k}\left(\left(x_{k} \sim x_{1}\right) \wedge \cdots \wedge\left(x_{k} \sim x_{k-1}\right)\right), \\
\phi_{2} & =\forall x_{1} \ldots \forall x_{k-2} \exists x_{k-1} \exists x_{k}\left(\left(x_{k-1} \sim x_{1}\right) \wedge\right. \\
& \wedge \wedge\left(x_{k-1} \sim x_{k-2}\right) \\
\wedge\left(x_{k} \sim x_{1}\right) & \left.\wedge \cdots\left(x_{k} \sim x_{k-2}\right) \wedge\left(x_{k-1} \sim x_{k}\right)\right) .
\end{aligned}
$$


Рассмотрим, кроме того, две пары графов $\left(G_{1}, H_{1}\right),\left(G_{2}, H_{2}\right)$, где $H_{1} \subset G_{1}$, $H_{2} \subset G_{2}, G_{1}$ и $G_{2}$ - полные графы на $k$ вершинах, $H_{1}$ - полный граф на $k-1$ вершинах, $H_{2}$ - полный граф на $k-2$ вершинах. Тогда при любых $\alpha<1 /(k-1)$ пара $\left(G_{1}, H_{1}\right)$ является $\alpha$-надежной, при любых $\alpha \in(1 /(k-1), 2 /(2 k-3))$ паpa $\left(G_{1}, H_{1}\right)$ является $\alpha$-жесткой, а пара $\left(G_{2}, H_{2}\right)-\alpha$-надежной, при любых $\alpha>2 /(2 k-3)$ пара $\left(G_{2}, H_{2}\right)$ является $\alpha$-жесткой. Очевидно, что граф обладает свойством $L_{1}$ тогда и только тогда, когда любой его подграф на $k-1$ вершине обладает $\left(G_{1}, H_{1}\right)$-расширением. Граф обладает свойством $L_{2}$ тогда и только тогда, когда любой его подграф на $k-2$ вершинах обладает $\left(G_{2}, H_{2}\right)$-расширением. В силу теоремы 13 при любых $\alpha<1 /(k-1)$ выполнено $\lim _{N \rightarrow \infty} \mathrm{P}_{N, N^{-\alpha}}\left(L_{1}\right)=1$, при любых $\alpha \in(1 /(k-1), 2 /(2 k-3))$ выполнено $\lim _{N \rightarrow \infty} \mathrm{P}_{N, N^{-\alpha}}\left(L_{1}\right)=0, \lim _{N \rightarrow \infty} \mathrm{P}_{N, N^{-\alpha}}\left(L_{2}\right)=1$, при любых $\alpha>2 /(2 k-3)$ выполнено $\lim _{N \rightarrow \infty} \mathrm{P}_{N, N^{-\alpha}}\left(L_{2}\right)=0$. Следовательно, $1 /(k-1), 2 /(2 k-3) \in \widetilde{S}_{k}$. Так как при $k \geqslant 3$ выполнено $1 /(k-2) \in S_{k}$, то и $1 /(k-2) \in \widetilde{S}_{k}$. Осталось доказать, что среди элементов множества $(0,1 /(k-1)) \cup(1 /(k-1), 2 /(2 k-3)) \cup$ $(2 /(2 k-3), 1 /(k-2))$ нет элементов множества $\widetilde{S}_{k}$. Для этого докажем критерий принадлежности числа спектру.

Лемма 5. Число $\alpha \in(0,1)$ не принадлежит спектру $\widetilde{S}_{k}$ тогда и только тогда, когда существует такое число $\varepsilon>0$, что для любых (не обязательно различных $) \alpha_{1}, \alpha_{2} \in(\alpha-\varepsilon, \alpha+\varepsilon)$ с вероятностью, стремящейся $\kappa 1$, у Консерватора есть выигрышная стратегия в игре $\operatorname{EHR}\left(G\left(N, N^{-\alpha_{1}}\right), G\left(M, M^{-\alpha_{2}}\right), k\right)$ при $N, M \rightarrow \infty$.

ДокАЗАТЕЛЬСтво. Предположим, что $\alpha \notin \widetilde{S}_{k}$. Тогда для любого свойства первого порядка $L$, выраженного формулой, кванторная глубина которой ограничена числом $k$, существует такое число $\varepsilon(L)>0$, что либо для любого $\alpha_{0} \in(\alpha-\varepsilon(L), \alpha+\varepsilon(L))$ выполнено равенство $\mathrm{P}_{N, N^{-\alpha_{0}}}(L)=0$, либо для любого $\alpha_{0} \in(\alpha-\varepsilon(L), \alpha+\varepsilon(L))$ выполнено равенство $\mathrm{P}_{N, N^{-\alpha_{0}}}(L)=1$. Обозначим $\mathscr{L}_{k}$ класс всех свойств первого порядка, выражаемых с помощью формул, кванторная глубина которых ограничена числом $k$. Положим $\varepsilon=\min _{L \in \mathscr{L}} \varepsilon(L)$. Тогда для любых $\alpha_{1}, \alpha_{2} \in(\alpha-\varepsilon, \alpha+\varepsilon)$ и для любого $L \in \mathscr{L}_{k}$ выполнено

$$
\lim _{N \rightarrow \infty} \mathrm{P}_{N, N^{-\alpha_{1}}}(L)=\lim _{N \rightarrow \infty} \mathrm{P}_{N, N^{-\alpha_{2}}}(L) \in\{0,1\} .
$$

Следовательно, случайный граф $G(N, p)$, где $p=N^{-\alpha_{1}}$, если $N$ - четно, и $p=N^{-\alpha_{2}}$, если $N$ - нечетно, подчиняется $k$-закону нуля или единицы. Тогда по теореме 21 с вероятностью, стремящейся к 1, у Консерватора есть выигрышная стратегия в игре $\operatorname{EHR}\left(G\left(N, N^{-\alpha_{1}}\right), G\left(M, M^{-\alpha_{2}}\right), k\right)$.

Пусть теперь $\varepsilon>0$ - такое число, что для любых $\alpha_{1}, \alpha_{2} \in(\alpha-\varepsilon, \alpha+\varepsilon)$ с вероятностью, стремящейся к 1, у Консерватора есть выигрышная стратегия в игре $\operatorname{EHR}\left(G\left(N, N^{-\alpha_{1}}\right), G\left(M, M^{-\alpha_{2}}\right), k\right)$ при $N, M \rightarrow \infty$. Пусть, кроме того, $L$ - произвольное свойство первого порядка. Рассмотрим случайный граф $G(N, p)$, где $p=N^{-\alpha_{1}}$, если $N$ - четно, и $p=N^{-\alpha_{2}}$, если $N$ - нечетно. Так как вероятности того, что у Консерватора есть выигрышные стратегии в играх $\operatorname{EHR}\left(G\left(N, N^{-\alpha_{1}}\right), G\left(M, M^{-\alpha_{2}}\right), k\right), \operatorname{EHR}\left(G\left(N, N^{-\alpha_{1}}\right), G\left(M, M^{-\alpha_{1}}\right), k\right)$, 
$\operatorname{EHR}\left(G\left(N, N^{-\alpha_{2}}\right), G\left(M, M^{-\alpha_{2}}\right), k\right)$, стремятся к 1 при $N, M \rightarrow \infty$, то стремится к 1 и вероятность того, что у Консерватора есть выигрышная стратегия в игpe $\operatorname{EHR}(G(N, p(N)), G(M, p(M)), k)$. Поэтому в силу теоремы 21 случайный граф $G(N, p)$ подчиняется $k$-закону нуля или единицы. Следовательно,

$$
\lim _{N \rightarrow \infty} \mathrm{P}_{N, N^{-\alpha_{1}}}(L)=\lim _{N \rightarrow \infty} \mathrm{P}_{N, N^{-\alpha_{2}}}(L) \in\{0,1\} .
$$

Лемма доказана.

Для завершения доказательства теоремы достаточно рассмотреть произвольное $\alpha \in(0,1 /(k-1)) \cup(1 /(k-1), 2 /(2 k-3)) \cup(2 /(2 k-3), 1 /(k-2))$ и такое $\varepsilon>0$, что

$$
(\alpha-\varepsilon, \alpha+\varepsilon) \subset\left(0, \frac{1}{k-1}\right) \cup\left(\frac{1}{k-1}, \frac{2}{2 k-3}\right) \cup\left(\frac{2}{2 k-3}, \frac{1}{k-2}\right),
$$

после чего, воспользовавшись для произвольных $\alpha_{1}, \alpha_{2} \in(\alpha-\varepsilon, \alpha+\varepsilon)$ рассуждениями из теоремы 22 , доказать, что у Консерватора с вероятностью, стремящейся к 1 , есть выигрышная стратегия в игре $\operatorname{EHR}\left(G\left(N, N^{-\alpha_{1}}\right), G\left(N, N^{-\alpha_{2}}\right), k\right)$.

\section{8. Дистанционные графы}

В этом разделе мы сделаем обзор законов нуля или единицы для свойств первого порядка случайных дистанционных графов, определение которых дано в разделе 2. Далее будем считать, что функция $p=p(N)$, где $N$ - количество вершин дистанционного графа, удовлетворяет условию (1).

В работах [35]-[39] были изучены законы нуля или единицы для случайных дистанционных графов, вершины которых являются векторами из $\{0,1\}^{n}$ с одинаковым количеством нулевых и единичных координат (в наших обозначениях $\left.M=\{0,1\}, a_{0}(n)=n / 2, a_{1}(n)=n / 2, c(n)=n / 4\right)$. Оказалось (см., например, [39]), что закон нуля или единицы для случайных дистанционных графов не выполнен. Однако в [35] были найдены подпоследовательности в рассматриваемой последовательности случайных дистанционных графов, подчиняющиеся закону нуля или единицы. Кроме того, в статье [38] были исследованы законы нуля или единицы для случайных дистанционных графов для формул первого порядка с ограниченной кванторной глубиной. Затем С.Н. Попова в [40] рассмотрела более общую модель - случайные дистанционные графы с вершинами в $\{-1,0,1\}^{n}$ (т.е. $M=\{-1,0,1\}$ ), зависящие от набора параметров $a_{-1}(n), a_{0}(n), a_{1}(n)$ и $c(n)$. Общий случай случайного дистанционного графа с вершинами в $\mathbb{Z}^{n}$ был рассмотрен С. Н. Поповой в [65]. В этой работе были получены условия, при которых последовательность случайных графов $\left\{\mathscr{G}\left(G_{n_{i}}^{M}, p\right)\right\}_{i \in \mathbb{N}}$ (см. раздел 2) подчиняется закону нуля или единицы, а также условия, при которых из упомянутой последовательности можно выделить подпоследовательность, подчиняющуюся этому закону. Прежде чем перейти к формулировкам и доказательствам этих результатов, мы мотивируем задачу, доказав, что в простейшем случае $M=\{0,1\}, a_{0}(n)=\alpha n, a_{1}(n)=(1-\alpha) n$, 
$c(n)=\alpha^{2} n$, где $\alpha-$ фиксированное рациональное число из $(0,1)$, случайный дистанционный граф не подчиняется закону нуля или единицы.

Для доказательств отрицательных и положительных результатов нам потребуются два вспомогательных утверждения. Пусть $M-$ произвольное конечное множество целых чисел, содержащее нуль, $\left\{\mathscr{G}\left(G_{n_{i}}^{M}, p\right)\right\}_{i \in \mathbb{N}}-$ последовательность случайных дистанционных графов. Введем вспомогательную величину $\Phi(n)$, выражаемую через параметры $a_{m}(n)$ и используемую в формулировках теорем:

$$
\Phi(n)=\sum_{m \in M} m a_{m}(n) .
$$

Заметим, что величина $\Phi(n)$ равна сумме всех координат произвольного вектора, являющегося вершиной дистанционного графа $G_{n}^{M}$. Для любого $t \in \mathbb{N}$ обозначим $L_{t}$ свойство графов для любых $t$ вершин содержать их общего соседа. Более того, скажем, что последовательность графов $\left\{G_{n_{i}}^{M}\right\}_{i \in \mathbb{N}}$ обладает свойcmвом $\widetilde{L}_{t}$, если существуют такие число $\beta>0$ и функция $\varphi(n, t)=\Omega\left(\left|V_{n}^{M}\right|^{\beta}\right)$, что для любых вершин $\mathbf{v}_{1}, \ldots, \mathbf{v}_{t} \in V_{n}^{M}$ в графе $G_{n}^{M}$ есть не менее $\varphi(n, t)$ вершин, соединенных ребрами с $\mathbf{v}_{1}, \ldots, \mathbf{v}_{t}$, при всех $n \in\left\{n_{i}\right\}_{i \in \mathbb{N}}$.

Итак, справедливо следующее утверждение.

УтвеРЖДЕнИе 1 (С.Н. Попова, 2013, [40]). Пусть при всех $n \in\left\{n_{i}\right\}_{i \in \mathbb{N}}$ выполнены равенства

$$
\Phi(n)=0, \quad c(n)=0
$$

и $a_{0}\left(n_{i}\right) \rightarrow \infty$ при $i \rightarrow \infty$. Тогда последовательность $\left\{G_{n_{i}}^{M}\right\}_{i \in \mathbb{N}}$ обладает свойством $\widetilde{L}_{t}$ при каждом $t \in \mathbb{N}$.

Прежде чем сформулировать второе утверждение, введем несколько обозначений. Обозначим $\mathscr{M}_{l \times h}$ множество всех матриц, элементы которых принадлежат множеству $M$, размера $l \times h$ и ранга $l$. Пусть $\Delta_{l}$ - максимальный среди определителей всех матриц из $\mathscr{M}_{l \times l}, D_{l}$ - наименьшее натуральное число, делящееся на все натуральные числа, не превосходящие $\Delta_{l}$, т. е. $D_{l}=\operatorname{HOK}\left(\Delta_{l}, \Delta_{l}-1, \ldots, 1\right)$. Введем множество $M_{+}=\{m \in M: m>0\}$ и обозначим $\eta$ наименьшее натуральное число, делящееся на все числа из множества $M_{+}$, т. е. $\eta=\operatorname{HOK}\left\{m: m \in M_{+}\right\}$.

УтвеРЖДЕНИе 2 (С.Н. Попова, 2014, [65]). Пусть при всех $n \in\left\{n_{i}\right\}_{i \in \mathbb{N}}$ выполнены равенства

$$
\Phi(n)=\alpha n, \quad c(n)=\alpha^{2} n,
$$

где $\alpha \in \mathbb{Q} \cap(0,1)$ - фиксированное число, и $a_{0}\left(n_{i}\right) \rightarrow \infty$ при $i \rightarrow \infty$. Пусть $t \in \mathbb{N}$ и последовательность $\left\{G_{n_{i_{j}}}\right\}_{j \in \mathbb{N}}$ такова, что числа $\Phi\left(n_{i_{j}}\right)$ и с $\left(n_{i_{j}}\right)$ делятся на $\eta \cdot D_{t+1}$ при достаточно больших $j \in \mathbb{N}$. Тогда последовательность $\left\{G_{n_{i_{j}}}^{M}\right\}_{j \in \mathbb{N}}$ обладает свойством $\widetilde{L}_{t}$.

Доказательства утверждений нетривиальны и техничны, поэтому в настоящем обзоре мы их не приводим. 
Вернемся к обещанной мотивировке. Пусть

$$
M=\{0,1\}, \quad a_{0}(n)=\alpha n, \quad a_{1}(n)=(1-\alpha) n, \quad c(n)=\alpha^{2} n,
$$

где $\alpha$ - фиксированное рациональное число из $(0,1)$. Докажем, что случайный дистанционный граф $\mathscr{G}\left(G_{n}^{M}, p\right)$ не подчиняется закону нуля или единицы. Этот результат является обобщением построенного в [35] примера существования свойства первого порядка, пределы вероятности которого различны для различных подпоследовательностей случайных дистанционных графов в случае $\alpha=1 / 2$. Пусть $\alpha=s / q$ - несократимая дробь и $q>2$ (случай $q=2$ был разобран в [35]). Пусть, кроме того, $n$ не делится на $q^{3}$. Пусть, например, $q<2 s$ (в противном случае пример строится аналогичным образом). Рассмотрим вершины $\mathbf{x}_{1}, \ldots, \mathbf{x}_{q^{2}+q-1} \in V_{n}^{M}$, определенные следующим образом:

\begin{tabular}{|c|c|}
\hline $\mathbf{x}_{1}$ & $=(1,1,1,1, \ldots, 1,1, \ldots, 1,1,1, \ldots, 1,1,0,0,0, \ldots, 0,0, \ldots, 0,0)$, \\
\hline & $=(\mathbf{0}, \mathbf{1}, \mathbf{1}, \mathbf{1}, \ldots, \mathbf{1}, \mathbf{1}, \ldots, \mathbf{1}, \mathbf{1}, \mathbf{1}, \ldots, \mathbf{1}, \mathbf{1}, \mathbf{1}, \mathbf{0}, \mathbf{0}, \ldots, \mathbf{0}, \mathbf{0}, \ldots, \mathbf{0}, \mathbf{0})$ \\
\hline $\mathbf{x}_{q^{2}-q s+1}$ & $=(\mathbf{0}, \mathbf{0}, \mathbf{0}, \mathbf{0}, \ldots, \mathbf{0}, \mathbf{0}, \ldots, \mathbf{0}, \mathbf{1}, \mathbf{1}, \ldots, \mathbf{1}, \mathbf{1}, \mathbf{1}, \mathbf{1}, \mathbf{1}, \ldots, \mathbf{1}, \mathbf{1}, \ldots, \mathbf{1}, \mathbf{1})$ \\
\hline $\mathbf{x}_{q^{2}-q s+2}$ & $=(1,0,0,0, \ldots, 0,0, \ldots, 0,0,1, \ldots, 1,1,1,1,1, \ldots, 1,1, \ldots, 1,1)$, \\
\hline $\mathbf{x}_{q^{2}}$ & $=(\mathbf{1}, \mathbf{1}, \mathbf{1}, \mathbf{1}, \ldots, \mathbf{1}, \mathbf{1}, \ldots, \mathbf{1}, \mathbf{1}, \mathbf{1}, \ldots, \mathbf{1}, \mathbf{0}, \mathbf{0}, \mathbf{0}, \mathbf{0}, \ldots, \mathbf{0}, \mathbf{0}, \ldots, \mathbf{0}, \mathbf{1})$ \\
\hline $\mathbf{x}_{q^{2}+1}$ & 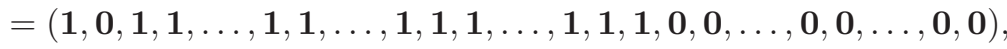 \\
\hline $\mathbf{x}_{q^{2}+2}$ & $=(\mathbf{1}, \mathbf{0}, \mathbf{0}, \mathbf{1}, \ldots, \mathbf{1}, \mathbf{1}, \ldots, \mathbf{1}, \mathbf{1}, \mathbf{1}, \ldots, \mathbf{1}, \mathbf{1}, \mathbf{1}, \mathbf{1}, \mathbf{0}, \ldots, \mathbf{0}, \mathbf{0}, \ldots, \mathbf{0}, \mathbf{0})$, \\
\hline $\mathbf{x}_{q^{2}+q-1}$ & $=(1,0,0,0, \ldots, 0,1, \ldots, 1,1,1, \ldots, 1,1,1,1,1, \ldots, 1,0, \ldots, 0,0)$ \\
\hline & $q^{2}-q s-q$ \\
\hline
\end{tabular}

Здесь 1 и $\mathbf{0}$ - векторы, составленные из $n / q^{2}$ единиц и $n / q^{2}$ нулей соответственно. Предположим, что существует вершина $\mathbf{x}_{q^{2}+q}$ в $V_{n}^{M}$, соединенная ребрами с каждой из $\mathbf{x}_{1}, \ldots, \mathbf{x}_{q^{2}+q-1}$. Обозначим $x_{1}$ количество единиц в векторе $\mathbf{x}_{q^{2}+q}$ среди первых $n / q^{2}$ координат, $x_{2}$ - среди следующих $n / q^{2}$ координат, и т. д. (получим $q^{2}$ чисел $\left.x_{1}, \ldots, x_{q^{2}}\right)$. Тогда вектор $\left(x_{1}, \ldots, x_{q^{2}}\right)$ является решением системы

$$
\begin{gathered}
x_{1}+\cdots+x_{s q}=\alpha^{2} n, \\
x_{2}+\cdots+x_{s q+1}=\alpha^{2} n, \\
\cdots \\
x_{\left(q^{2}-s q\right)+1}+\cdots+x_{q^{2}}=\alpha^{2} n, \\
x_{1}+x_{\left(q^{2}-s q\right)+2}+\cdots+x_{q^{2}}=\alpha^{2} n, \\
\cdots \\
x_{1}+\cdots+x_{s q-1}+x_{q^{2}}=\alpha^{2} n, \\
x_{1}+x_{3}+x_{4}+\cdots+x_{s q}+x_{s q+1}=\alpha^{2} n, \\
x_{1}+x_{4}+x_{5}+\cdots+x_{s q+1}+x_{s q+2}=\alpha^{2} n, \\
\cdots \\
x_{1}+x_{q+1}+x_{q+2}+\cdots+x_{s q+q-2}+x_{s q+q-1}=\alpha^{2} n .
\end{gathered}
$$


Из первых $q^{2}$ уравнений системы получаем, что

$$
\begin{aligned}
& x_{1}=x_{1+q r_{1}}=x_{1+q r_{2}}=\cdots=x_{1+q r_{q-1}}, \\
& x_{2}=x_{2+q r_{1}}=x_{2+q r_{2}}=\cdots=x_{2+q r_{q-1}}, \\
& \ldots \ldots \ldots \ldots \ldots \cdots \cdots \cdots \\
& x_{q}=x_{q+q r_{1}}=x_{q+q r_{2}}=\cdots=x_{q+q r_{q-1}},
\end{aligned}
$$

где $r_{i}, i \in\{1, \ldots, q-1\},-$ остатки от деления $i s$ на $q$. Так как числа $s$ и $q$ взаимно просты, то все числа $r_{1}, \ldots, r_{q-1}$ различны и отличны от 0. Поэтому $x_{1}=x_{q+1}=\cdots=x_{q^{2}-q+1}, x_{2}=x_{q+2}=\cdots=x_{q^{2}-q+2}, \ldots, x_{q}=x_{2 q}=\cdots=$ $x_{q^{2}}$. Из 2-го, 3-го, $\ldots, q$-го и последних $q-1$ уравнений получаем равенство $x_{1}=\cdots=x_{q}$. Следовательно, $x_{1}=\cdots=x_{q^{2}}=\alpha^{2} n /(q s)=s n / q^{3}$, но $s$ и $q$ взаимно просты, а $n$ не делится на $q^{3}$. Получили противоречие. Таким образом, не существует вершины $\mathbf{x}_{q^{2}+q}$ в $V_{n}^{M}$, соединенной ребрами с каждой из $\mathbf{x}_{1}, \ldots, \mathbf{x}_{q^{2}+q-1}$. Следовательно, граф $G_{n}^{M}$ не обладает свойством $L_{q^{2}+q-1}$.

Рассмотрим последовательности натуральных чисел $\left\{t_{i}\right\}_{i \in \mathbb{N}}$ и $\left\{m_{i}\right\}_{i \in \mathbb{N}}$, где $t_{i}=i q^{3}+q^{2}$ и для любого $a \in \mathbb{N}$ существует такое $i_{0}$, что при $i \geqslant i_{0}$ число $m_{i}$ делится на $a$. Составим из этих двух последовательностей одну: $n_{2 i}=m_{i}$, $n_{2 i-1}=t_{i}$. Тогда, очевидно,

$$
\lim _{i \rightarrow \infty} \mathrm{P}_{G_{n_{2 i-1}, p}^{M}}\left(L_{q^{2}+q-1}\right)=0 .
$$

Докажем, что

$$
\lim _{i \rightarrow \infty} \mathrm{P}_{G_{n_{2 i}}^{M}, p}\left(L_{q^{2}+q-1}\right)=1,
$$

что и послужит опровержением закона нуля или единицы.

По утверждению 2 последовательность $\left\{G_{n_{2 i}}^{M}\right\}_{i \in \mathbb{N}}$ обладает свойством $\widetilde{L}_{q^{2}+q-1}$. Таким образом, существуют такие число $\beta>0$ и функция $\varphi(n)=\Omega\left(\left|V_{n}^{M}\right|^{\beta}\right)$, что для любого $n \in\left\{n_{2 i}, i \in \mathbb{N}\right\}$ и любых вершин $\mathbf{x}_{1}, \ldots, \mathbf{x}_{q^{2}+q-1} \in V_{n}^{M}$ в графе $G_{n}^{M}$ есть не менее $\varphi(n)$ вершин, соединенных ребрами с $\mathbf{x}_{1}, \ldots, \mathbf{x}_{q^{2}+q-1}$. Тогда в силу (1)

$$
\mathrm{P}_{G_{n}^{M}, p}\left(\overline{L_{q^{2}+q-1}}\right) \leqslant N^{q^{2}+q-1}\left(1-p(n)^{q^{2}+q-1}\right)^{\varphi(n)} \rightarrow 0, \quad i \rightarrow \infty .
$$

Поэтому, действительно, $\lim _{i \rightarrow \infty} \mathrm{P}_{G_{n_{2 i}}^{M}}\left(L_{q^{2}+q-1}\right)=1$, и, следовательно, случайный граф $\mathscr{G}\left(G_{n}^{M}, p\right)$ не подчиняется закону нуля или единицы.

Обратимся теперь к условиям, при которых случайных дистанционный граф подчиняется закону нуля или единицы. В следующей теореме даются ограничения на функции $a_{m}(n), m \in M$, и $c(n)$, при которых последовательность случайных графов $\mathscr{G}\left(G_{n_{i}}^{M}, p\right)$ подчиняется закону нуля или единицы.

Tеорема 27 (С. Н. Попова, 2014, [65]). Пусть $\widetilde{m} \in M$, при всех $n \in\left\{n_{i}\right\}_{i \in \mathbb{N}}$ выполнены равенства

$$
\Phi(n)=\widetilde{m} \cdot n, \quad c(n)=\widetilde{m}^{2} \cdot n
$$

и $a_{\widetilde{m}}\left(n_{i}\right) \rightarrow \infty$ при $i \rightarrow \infty$. Тогда последовательность $\left\{\mathscr{G}\left(G_{n_{i}}^{M}, p\right)\right\}_{i \in \mathbb{N}}$ подчиняется закону нуля или единицы. 
ДокАЗАТЕЛЬСтво. Сначала покажем, что при любых значениях параметров $a_{m}(n), m \in M$, и $c(n)$ и при любом $\mu \in \mathbb{Z}$ граф $G_{n}^{M}$ изоморфен графу $G_{n}^{M-\mu}$, задаваемому параметрами

$$
\widetilde{a}_{l}(n)=a_{l+\mu}(n), \quad l \in M-\mu, \quad \text { и } \quad \widetilde{c}(n)=c(n)-2 \mu \Phi(n)+\mu^{2} n,
$$

где $M-\mu=\{m-\mu: m \in M\}$. Определим отображение $\varphi: V\left(G_{n}^{M}\right) \rightarrow V\left(G_{n}^{M-\mu}\right)$ следующим образом:

$$
\varphi\left(\left(v^{1}, \ldots, v^{n}\right)\right)=\left(v^{1}-\mu, \ldots, v^{n}-\mu\right) .
$$

Тогда

$$
\langle\varphi(\mathbf{u}), \varphi(\mathbf{v})\rangle=\langle\mathbf{u}, \mathbf{v}\rangle-2 \mu \sum_{i=1}^{n} v^{i}+\mu^{2} n=\langle\mathbf{u}, \mathbf{v}\rangle-2 \mu \Phi(n)+\mu^{2} n
$$

Поэтому

$$
\{\mathbf{u}, \mathbf{v}\} \in E\left(G_{n}^{M}\right) \Leftrightarrow\langle\mathbf{u}, \mathbf{v}\rangle=c \Leftrightarrow\langle\varphi(\mathbf{u}), \varphi(\mathbf{v})\rangle=\widetilde{c} \Leftrightarrow\{\varphi(\mathbf{u}), \varphi(\mathbf{v})\} \in E\left(G_{n}^{M-\mu}\right) .
$$

Следовательно, $\varphi$ - изоморфизм графов $G_{n}^{M}$ и $G_{n}^{M-\mu}$.

Перейдем теперь к рассмотрению графа $G_{n}^{M}$ с параметрами, удовлетворяющими условиям $\Phi(n)=\widetilde{m} \cdot n, c(n)=\widetilde{m}^{2} \cdot n$ при всех $n \in\left\{n_{i}\right\}_{i \in \mathbb{N}}$ и $a_{\widetilde{m}}\left(n_{i}\right) \rightarrow \infty$ при $i \rightarrow \infty$. Выбрав в качестве $\mu$ число $\widetilde{m}$, получим, что такой граф $G_{n}^{M}$ изоморфен графу $G_{n}^{M-\mu}$, для которого $\Phi(n)=0, c(n)=0$ при всех $n \in\left\{n_{i}\right\}_{i \in \mathbb{N}}$ и $a_{0}\left(n_{i}\right) \rightarrow \infty$ при $i \rightarrow \infty$.

Докажем, что для каждого $t \in \mathbb{N}$ случайный граф $\mathscr{G}\left(G_{n}^{M}, p\right), n \in\left\{n_{i}\right\}_{i \in \mathbb{N}}$, обладает свойством полного расширения уровня $t$ с асимптотической вероятностью 1. Пусть $E_{t}$ - событие, состоящее в том, что случайный граф $\mathscr{G}\left(G_{n}^{M}, p\right)$, $n \in\left\{n_{i}\right\}_{i \in \mathbb{N}}$, не обладает свойством полного расширения уровня $t$. Для различ-

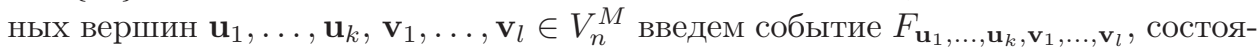
щее в том, что в случайном графе $\mathscr{G}\left(G_{n}^{M}, p\right)$ не найдется вершины, соединенной ребрами с $\mathbf{u}_{1}, \ldots, \mathbf{u}_{k}$ и не соединенной ребрами с $\mathbf{v}_{1}, \ldots, \mathbf{v}_{l}$. Заметим, что

$$
E_{t}=\bigcup_{\substack{k, l \in \mathbb{Z}_{+} \\ k+l \leqslant t}} \bigcup_{\mathbf{u}_{1}, \ldots, \mathbf{u}_{k}, \mathbf{v}_{1}, \ldots, \mathbf{v}_{l} \in V_{n}^{M}} F_{\mathbf{u}_{1}, \ldots, \mathbf{u}_{k}, \mathbf{v}_{1}, \ldots, \mathbf{v}_{l}} .
$$

Из утверждения 1 следует, что существуют такое $\beta=\beta(t)>0$ и такая функция $\varphi(n, t)=\Omega\left(\left|V_{n}^{M}\right|^{\beta}\right)$, что для любых вершин $\mathbf{u}_{1}, \ldots, \mathbf{u}_{k}, \mathbf{v}_{1}, \ldots, \mathbf{v}_{l} \in V_{n}^{M}$, где $k, l \in \mathbb{Z}_{+}, k+l \leqslant t$, в графе $G_{n}^{M}$ есть не менее $\varphi(n, t)$ вершин, соединенных ребрами с $\mathbf{u}_{1}, \ldots, \mathbf{u}_{k}, \mathbf{v}_{1}, \ldots, \mathbf{v}_{l}$. Тогда

$$
\mathrm{P}_{G_{n}^{M}, p}\left(F_{\mathbf{u}_{1}, \ldots, \mathbf{u}_{k}, \mathbf{v}_{1}, \ldots, \mathbf{v}_{l}}\right) \leqslant\left(1-\varepsilon(n)^{t}\right)^{\Omega\left(\left|V_{n}^{M}\right|^{\beta}\right)},
$$

где

$$
\varepsilon(n)=\min \left\{p\left(\left|V_{n}^{M}\right|\right), 1-p\left(\left|V_{n}^{M}\right|\right)\right\}
$$


Поэтому

$$
\mathrm{P}_{G_{n_{i}}^{M}, p}\left(E_{t}\right) \leqslant t^{2}\left|V_{n_{i}}^{M}\right|^{t}\left(1-\varepsilon\left(n_{i}\right)^{t}\right)^{\Omega\left(\left|V_{n_{i}}^{M}\right|^{\beta}\right)} \leqslant t^{2}\left|V_{n_{i}}^{M}\right|^{t} \exp \left\{-\varepsilon\left(n_{i}\right)^{t} \Omega\left(\left|V_{n_{i}}^{M}\right|^{\beta}\right)\right\} .
$$

Последнее выражение стремится к 0 при $i \rightarrow \infty$ в силу условия (1) на функцию $p$. Следовательно, случайный граф $\mathscr{G}\left(G_{n_{i}}^{M}, p\right)$ с вероятностью, стремящейся к единице, обладает свойством полного расширения уровня $t$, и потому последовательность $\left\{\mathscr{G}\left(G_{n_{i}}^{M}, p\right)\right\}_{i \in \mathbb{N}}$ подчиняется закону нуля или единицы. Теорема доказана.

Сформулируем теперь условия, при которых можно найти подпоследовательность случайных дистанционных графов, подчиняющуюся закону нуля или единицы.

Tеорема 28 (С. Н. Попова, 2014, [65]). Пусть $\widetilde{m} \in M$, при всех $n \in\left\{n_{i}\right\}_{i \in \mathbb{N}}$ выполнены равенства

$$
\Phi(n)=(\widetilde{m}+\alpha) n, \quad c(n)=(\widetilde{m}+\alpha)^{2} n,
$$

где $\alpha \in \mathbb{Q} \cap(0,1)$ - фиксированное число, и $a_{\widetilde{m}}\left(n_{i}\right) \rightarrow \infty$ nри $i \rightarrow \infty$. Пусть, кроме того, подпоследовательность $\left\{n_{i_{j}}\right\}_{j \in \mathbb{N}}$ такова, что для любого $d \in \mathbb{N}$ существует такое $j_{0} \in \mathbb{N}$, что при $j>j_{0}$ числа $n_{i_{j}}$ делятся на $d$. Тогда последовательность $\left\{\mathscr{G}\left(G_{n_{i_{j}}}^{M}, p\right)\right\}_{j \in \mathbb{N}}$ подчиняется закону нуля или единиць.

ДокАзАтельство. Пусть $\left\{n_{i_{j}}\right\}_{j \in \mathbb{N}}-$ подпоследовательность, удовлетворяющая условию теоремы. Учитывая, что граф $G_{n}^{M}$ изоморфен графу $G_{n}^{\widetilde{M}}$, для которого $\Phi(n)=\alpha n, c(n)=\alpha^{2} n$ при всех $n \in\left\{n_{i}\right\}_{i \in \mathbb{N}}$ и $a_{0}\left(n_{i}\right) \rightarrow \infty$ при $i \rightarrow \infty$, и применяя утверждение 2 , получаем, что существуют такое $\beta=\beta(t)>0$ и такая функция $\varphi(n, t)=\Omega\left(\left|V_{n}^{M}\right|^{\beta}\right)$, что при всех $n \in\left\{n_{i_{j}}\right\}_{j \in \mathbb{N}}$ для любых вершин $\mathbf{v}_{1}, \ldots, \mathbf{v}_{t} \in V_{n}^{M}$ в графе $G_{n}^{M}$ есть не менее $\varphi(n, t)$ вершин, соединенных ребрами с $\mathbf{v}_{1}, \ldots, \mathbf{v}_{t}$. Оценивая вероятность отсутствия свойства полного расширения уровня $t$ таким же образом, как и в доказательстве теоремы 27 , заключаем, что последовательность $\left\{\mathscr{G}\left(G_{n_{i_{j}}}, p\right)\right\}_{j \in \mathbb{N}}$ подчиняется закону нуля или единицы. Теорема доказана.

Вернемся к случайному графу $\mathscr{G}\left(G_{n}^{\{0,1\}}, p\right)$, определенному следующим образом: $a_{0}(n)=\alpha n, a_{1}(n)=(1-\alpha) n, c(n)=\alpha^{2} n, \alpha=s / q-$ несократимая дробь, $0<s<q$. Как уже было замечено выше, он не подчиняется закону нуля или единицы. Тем не менее из теоремы 28 следует, что во всей последовательности рассматриваемых случайных дистанционных графов $\left\{\mathscr{G}\left(G_{q^{2} i}^{\{0,1\}}, p\right)\right\}_{i \in \mathbb{N}}$ существует подпоследовательность, подчиняющаяся этому закону.

\section{Список литературы}

[1] В. Л. Гончаров, "О распределении циклов в перестановках", Докл. АН СССР, 35:9 (1942), 299-301.

[2] T. Szele, "Kombinatorikai vizsgálatok az irányitott teljes gráffal kapcsolatban", Mat. Fiz. Lapok, 50 (1943), 223-256. 
[3] P. Erdős, "Graph theory and probability", Canad. J. Math., 11 (1959), 34-38.

[4] P. Erdős, A. Rényi, "On random graphs. I", Publ. Math. Debrecen, 6 (1959), 290-297.

[5] P. Erdős, A. Rényi, "On the evolution of random graphs", Magyar Tud. Akad. Mat. Kutató Int. Közl., 5 (1960), 17-61.

[6] P. Erdős, A. Rényi, "On the evolution of random graphs", Bull. Inst. Internat. Statist., 38 (1961), 343-347.

[7] В. Ф. Колчин, Случайные графы, 2-е изд., Физматлит, М., 2004, 256 с.; англ. изд.: V. F. Kolchin, Random graphs, Encyclopedia Math. Appl., 53, Cambridge Univ. Press, Cambridge, 1999, xii+252 pp.

[8] N. Alon, J. H. Spencer, The probabilistic method, 3rd ed., Wiley-Intersci. Ser. Discrete Math. Optim., John Wiley \& Sons, Inc., Hoboken, NJ, 2008, xviii+352 pp.

[9] B. Bollobás, Random graphs, 2nd ed., Cambridge Stud. Adv. Math., 73, Cambridge Univ. Press, Cambridge, 2001, xviii+498 pp.

[10] S. Janson, T. Łuczak, A. Ruciński, Random graphs, Wiley-Intersci. Ser. Discrete Math. Optim., Wiley-Interscience, New York, 2000, xii+333 pp.

[11] А. М. Райгородский, Модели случайных графов, МЦНМО, М., 2011, 136 с.

[12] А. М. Райгородский, Модели интернета, Интеллект, Долгопрудный, 2013, 64 с.

[13] L. Lovász, Large networks and graph limits, Amer. Math. Soc. Colloq. Publ., 60, Amer. Math. Soc., Providence, RI, 2012, xiv+475 pp.

[14] S. N. Dorogovtsev, Lectures on complex networks, Oxf. Master Ser. Phys., 20, Oxford Univ. Press, Oxford, 2010, $\mathrm{x}+134 \mathrm{pp}$.

[15] M. Penrose, Random geometric graphs, Oxford Stud. Probab., 5, Oxford Univ. Press, Oxford, 2003, xiv+330 pp.

[16] M.E. J. Newman, Networks. An introduction, Oxford Univ. Press, Oxford, 2010, xii+772 pp.

[17] B. Bollobás, A. Thomason, "Threshold functions", Combinatorica, 7:1 (1987), 35-38.

[18] A. Ruciński, A. Vince, "Balanced graphs and the problem of subgraphs of a random graph", Proceedings of the Sixteenth Southeastern International Conference on Combinatorics, Graph Theory and Computing (Boca Raton, FL, 1985), Congr. Numer., 49 (1985), 181-190.

[19] B. Bollobás, "Threshold functions for small subgraphs", Math. Proc. Cambridge Philos. Soc., 90:2 (1981), 197-206.

[20] A. Ruciński, A. Vince, "Strongly balanced graphs and random graphs", J. Graph Theory, 10:2 (1986), 251-264.

[21] А. М. Райгородский, Линейно-алгебрачческий метод в комбинаторике, МЦНМО, M., 2007, 138 с.

[22] А.М. Райгородский, "Проблема Борсука и хроматические числа некоторых метрических пространств", УМН, 56:1(337) (2001), 107-146; англ. пер.: A. M. Raigorodskii, "Borsuk's problem and the chromatic numbers of some metric spaces", Russian Math. Surveys, 56:1 (2001), 103-139.

[23] A. M. Raigorodskii, "Coloring distance graphs and graphs of diameters", Thirty essays on geometric graph theory, ed. J. Pach, Springer, New York, 2013, 429-460.

[24] J. Pach, P. K. Agarwal, Combinatorial geometry, Wiley-Intersci. Ser. Discrete Math. Optim., John Wiley \& Sons, Inc., New York, 1995, xiv+354 pp.

[25] L. A. Székely, "Erdős on unit distances and the Szemerédi-Trotter theorems", Paul Erdös and his mathematics, v. II (Budapest, 1999), Bolyai Soc. Math. Stud., 11, Budapest, 2002, 649-666. 
[26] A. Soifer, The mathematical coloring book. Mathematics of coloring and the colorful life of its creators, Springer, New York, 2009, xxx+607 pp.

[27] V. Klee, S. Wagon, Old and new unsolved problems in plane geometry and number theory, Dolciani Math. Exp., 11, Math. Assoc. America, Washington, DC, 1991, xvi+333 pp.

[28] A. M. Raigorodskii, "Cliques and cycles in distance graphs and graphs of diameters", Discrete geometry and algebraic combinatorics, Contemp. Math., 625, Amer. Math. Soc., Providence, RI, 2014, 93-109.

[29] P. Frankl, R. M. Wilson, "Intersection theorems with geometric consequences", Combinatorica, 1:4 (1981), 357-368.

[30] J. Kahn, G. Kalai, "A counterexample to Borsuk's conjecture", Bull. Amer. Math. Soc. (N. S.), 29:1 (1993), 60-62.

[31] P. Brass, W. Moser, J. Pach, Research problems in discrete geometry, Springer, New York, 2005, xii+499 pp.

[32] Ф. Дж. Мак-Вильямс, Н. Дж. А. Слоэн, Теория кодов, исправляющих ошибки, Связь, M., 1979, 744 с.; пер. с англ.: F. J. MacWilliams, N. J. A. Sloane, The theory of error-correcting codes. Parts I, II, North-Holland Mathematical Library, 16, North-Holland Publishing Co., Amsterdam-New York-Oxford, 1977, xv+ix+762 pp.

[33] V. Rödl, "On a packing and covering problem", European J. Combin., 6:1 (1985), 69-78.

[34] L. Bassalygo, G. Cohen, G. Zémor, "Codes with forbidden distances", Selected topics in discrete mathematics (Warsaw, 1996), Discrete Math., 213:1-3 (2000), 3-11.

[35] М.Е. Жуковский, "О последовательности случайных дистанционных графов, подчиняющейся закону нуля или единицы", Пробл. передачи информ., 47:3 (2011), 39-57; англ. пер.: M.E. Zhukovskii, "On a sequence of random distance graphs subject to the zero-one law", Problems Inform. Transmission, 47:3 (2011), 251-268.

[36] М.Е. Жуковский, "Ослабленный закон нуля или единицы для случайных дистанционных графов", Теория вероятн. и ее примен., 55:2 (2010), 344-349; англ. пер.: М.E. Zhukovskii, "The weak zero-one law for the random distance graphs", Theory Probab. Appl., 55:2 (2011), 356-360.

[37] М. Е. Жуковский, "Ослабленный закон нуля или единицы для случайных дистанционных графов”, Докл. РАН, 430:3 (2010), 314-317; англ. пер.: M. E. Zhukovskii, "Weak zero-one laws for random distance graph", Dokl. Math., 81:1 (2010), 51-54.

[38] М.Е. Жуковский, "Ослабленный закон нуля или единицы для последовательностей случайных дистанционных графов”, Матем. сб., 203:7 (2012), 95-128; англ. пер.: M. E. Zhukovskii, "A weak zero-one law for sequences of random distance graphs", Sb. Math., 203:7 (2012), 1012-1044.

[39] М.Е. Жуковский, “Ослабленный закон 'нуля или единицы' для случайных дистанционных графов", Вестн. РУДН, 2:1 (2010), 11-25.

[40] С.Н. Попова, "Закон нуля или единицы для случайных дистанционных графов с вершинами в $\{-1,0,1\}^{n}$ ", Пробл. передачи информ., 50:1 (2014), 64-86; англ. пер.: S. N. Popova, "Zero-one law for random distance graphs with vertices in $\{-1,0,1\}^{n}$ ", Problems Inform. Transmission, 50:1 (2014), 57-78.

[41] М.Е. Жуковский, "О вероятности вхождения копии фиксированного графа в случайный дистанционный граф”, Матем. заметки, 92:6 (2012), 844-855; англ. пер.: М. E. Zhukovskii, "On the probability of the occurrence of a copy of a fixed graph in a random distance graph", Math. Notes, 92:6 (2012), 756-766. 
[42] Л.И. Боголюбский, А. С. Гусев, М.М.Пядеркин, А. М. Райгородский, “Числа независимости и хроматические числа случайных подграфов в некоторых последовательностях графов", Докл. РАН, 457:4 (2014), 383-387.

[43] Л. И. Боголюбский, А.С. Гусев, М.М. Пядеркин, А. М. Райгородский, “Числа независимости и хроматические числа случайных подграфов некоторых дистанционных графов", Матем. сб. (в печати).

[44] A. B. Kupavskii, "On random subgraphs of Kneser graph", J. Combin. Theory Ser. A (to appear).

[45] B. Bollobás, B.P. Narayanan, A. M. Raigorodskii, "On the stability of the Erdős-Ko-Rado theorem", J. Combin. Theory Ser. A (to appear).

[46] Н. К. Верещагин, А. Шень, Языки и исчисления, МЦНМО, М., 2000, 286 с.

[47] В. А. Успенский, Н.К. Верещагин, В. Е. Плиско, Вводный курс математической логики, Физматлит, М., 2007, 128 с.

[48] A. Ehrenfeucht, "An application of games to the completness problem for formalized theories", Fund. Math., 49 (1960/1961), 121-141.

[49] J. Spencer, The strange logic of random graphs, Algorithms Combin., 22, SpringerVerlag, Berlin, 2001, $\mathrm{x}+168$ pp.

[50] S. Shelah, J. Spencer, "Zero-one laws for sparse random graphs", J. Amer. Math. Soc., 1:1 (1988), 97-115.

[51] Ю.В.Глебский, Д. И. Коган, М.И.Лиогонький, В.А. Таланов, “Объем и доля выполнимости формул узкого исчисления предикатов", Кибернетика, 2 (1969), $17-27$.

[52] R. Fagin, "Probabilities in finite models", J. Symbolic Logic, 41:1 (1976), 50-58.

[53] B. Kreuter, "Threshold functions for asymmetric Ramsey properties with repect to vertex colorings", Random Structures Algorithms, 9:3 (1996), 335-348.

[54] J. Spencer, "Counting extensions", J. Combin. Theory Ser. A, 55:2 (1990), 247-255.

[55] T. Łuczak, J. Spencer, "When does the zero-one law hold?", J. Amer. Math. Soc., 4:3 (1991), 451-468.

[56] J.F. Lynch, "Probabilities of sentences about very sparse random graphs", Random Structures Algorithms, 3:1 (1992), 33-53.

[57] M. McArthur, "The asymptotic behavior of $L_{\infty, \omega}^{k}$ on sparse random graphs", Logic and random structures (New Brunswick, NJ, 1995), DIMACS Ser. Discrete Math. Theoret. Comput. Sci., 33, Amer. Math. Soc., Providence, RI, 1997, 53-63.

[58] Ph. G. Kolaitis, H. J. Prömel, B. L. Rothschild, " $K_{l+1}$-free graphs: asymptotic structure and a 0-1 law", Trans. Amer. Math. Soc., 303:2 (1987), 637-671.

[59] R. H. Gilman, Y. Gurevich, A. Miasnikov, "A geometric zero-one law", 2007, 13 pp., arXiv: 0706.0271.

[60] M. Zhukovskii, "Zero-one k-law", Discrete Math., 312:10 (2012), 1670-1688.

[61] М.Е. Жуковский, "Оценка количества максимальных расширений в случайном графе", Дискрет. матем., 24:1 (2012), 79-107; англ. пер.: M. E. Zhukovskii, "Estimation of the number of maximal extensions in a random graph", Discrete Math. Appl., 22:1 (2012), 55-90.

[62] М.Е. Жуковский, "Расширение $k$-закона нуля или единицы”, Докл. PAH, 454:1 (2014), 23-26; англ. пер.: M. E. Zhukovskii, "Extension of the zero-one $k$-law", Dokl. Math., 89:1 (2014), 16-19.

[63] М. Е. Жуковский, "О наибольшей критической точке в $k$-законе нуля или единицы”, Матем. сб. (в печати). 
[64] J. Spencer, "Infinite spectra in the first order theory of graphs", Combinatorica, 10:1 (1990), 95-102.

[65] С.Н. Попова, “Закон нуля или единицы для случайных дистанционных графов с вершинами в $\mathbb{Z}^{n "}$, Матем. сб. (в печати).

\section{Максим Евгеньевич Жуковский}

(Maksim E. Zhukovskii)

Московский физико-технический институт (государственный университет)

E-mail: zhukmax@gmail.com

\section{Андрей Михайлович Райгородский}

(Andrei M. Raigorodskii)

Московский государственный университет им. М. В. Ломоносова;

Московский физико-технический институт (государственный университет)

E-mail: mraigor@yandex.ru
Поступила в редакцию

05.09.2014 